\title{
Three-Higgs-doublet models: symmetries, potentials and Higgs boson masses
}

\author{
Venus Keus, ${ }^{a, b, c}$ Stephen F. King ${ }^{a}$ and Stefano Moretti ${ }^{a, c}$ \\ ${ }^{a}$ School of Physics and Astronomy, University of Southampton, \\ Southampton, SO17 1BJ, U.K. \\ ${ }^{b}$ Department of Physics, Royal Holloway, University of London, \\ Egham Hill, Egham TW20 0EX, U.K. \\ ${ }^{c}$ Particle Physics Department, Rutherford Appleton Laboratory, \\ Chilton, Didcot, Oxon OX11 0QX, U.K. \\ E-mail: V.Keus@soton.ac.uk, king@soton.ac.uk, S.Moretti@soton.ac.uk
}

ABStRaCT: We catalogue and study three-Higgs-doublet models in terms of all possible allowed symmetries (continuous and discrete, Abelian and non-Abelian), where such symmetries may be identified as flavour symmetries of quarks and leptons. We analyse the potential in each case, and derive the conditions under which the vacuum alignments $(0,0, v)$, $(0, v, v)$ and $(v, v, v)$ are minima of the potential. For the alignment $(0,0, v)$, relevant for dark matter models, we calculate the corresponding physical Higgs boson mass spectrum. Motivated by supersymmetry, we extend the analysis to the case of three up-type Higgs doublets and three down-type Higgs doublets (six doublets in total). Many of the results are also applicable to flavon models where the three Higgs doublets are replaced by three electroweak singlets.

Keywords: Higgs Physics, Spontaneous Symmetry Breaking, Beyond Standard Model, Discrete and Finite Symmetries

ARXIV EPRINT: 1310.8253 


\section{Contents}

1 Introduction 1

2 Symmetries and symmetry breaking in 3HDMs 3

3 Analysis of Abelian 3HDMs $\quad 6$

$3.1 \mathrm{U}(1) \times \mathrm{U}(1)$ symmetric $3 \mathrm{HDM}$ potential $\quad 6$

$\begin{array}{llr}3.2 & \mathrm{U}(1) & \text { symmetric } 3 \mathrm{HDM} \text { potential }\end{array}$

$\begin{array}{lll}3.3 & \mathrm{U}(1) \times \mathrm{Z}_{2} \text { symmetric 3HDM potential } & 11\end{array}$

$\begin{array}{lll}3.4 & \mathrm{Z}_{2} \text { symmetric } 3 \mathrm{HDM} \text { potential } & 13\end{array}$

$\begin{array}{lll}3.5 & \mathrm{Z}_{3} \text { symmetric } 3 \mathrm{HDM} \text { potential } & 15\end{array}$

$\begin{array}{lll}3.6 & \mathrm{Z}_{4} \text { symmetric } 3 \mathrm{HDM} \text { potential } & 17\end{array}$

$\begin{array}{lll}3.7 & \mathrm{Z}_{2} \times \mathrm{Z}_{2} \text { symmetric } 3 \mathrm{HDM} \text { potential } & 18\end{array}$

4 Analysis of non-Abelian finite 3HDM $\quad 20$

$4.1 \mathrm{D}_{6} \simeq \mathrm{Z}_{3} \rtimes \mathrm{Z}_{2}$ symmetric $3 \mathrm{HDM}$ potential $\quad 20$

$4.2 \mathrm{D}_{8} \simeq \mathrm{Z}_{4} \rtimes \mathrm{Z}_{2}$ symmetric 3HDM potential $\quad 22$

$4.3 \mathrm{~A}_{4} \simeq \mathrm{T}=\left(\mathrm{Z}_{2} \times \mathrm{Z}_{2}\right) \rtimes \mathrm{Z}_{3}$ symmetric $3 \mathrm{HDM}$ potential 24

$4.4 \mathrm{~S}_{4} \simeq \mathrm{O}=\left(\mathrm{Z}_{2} \times \mathrm{Z}_{2}\right) \rtimes \mathrm{S}_{3}$ symmetric $3 \mathrm{HDM}$ potential 25

$4.5 \Delta(54) / \mathrm{Z}_{3} \simeq\left(\mathrm{Z}_{3} \times \mathrm{Z}_{3}\right) \rtimes \mathrm{Z}_{2}$ symmetric 3HDM potential 27

$4.6 \Sigma(36) \simeq\left(\mathrm{Z}_{3} \times \mathrm{Z}_{3}\right) \rtimes \mathrm{Z}_{4}$ symmetric $3 \mathrm{HDM}$ potential 29

5 Analysis of 6HDMs $\quad 31$

$5.1 \mathrm{U}(1) \times \mathrm{U}(1)$ symmetric 6 HDM potential 31

$5.2 \mathrm{U}(1)$ symmetric $6 \mathrm{HDM}$ potential 32

$5.3 \mathrm{U}(1) \times \mathrm{Z}_{2}$ symmetric $6 \mathrm{HDM}$ potential 33

$5.4 \mathrm{Z}_{2}$ symmetric $6 \mathrm{HDM}$ potential $\quad 34$

$5.5 \mathrm{Z}_{3}$ symmetric 6HDM potential 36

$\begin{array}{lll}5.6 & \mathrm{Z}_{4} \text { symmetric } 6 \mathrm{HDM} \text { potential } & 36\end{array}$

$5.7 \mathrm{Z}_{2} \times \mathrm{Z}_{2}$ symmetric $6 \mathrm{HDM}$ potential $\quad 36$

$5.8 \mathrm{D}_{6}$ symmetric $6 \mathrm{HDM}$ potential $\quad 37$

$\begin{array}{lll}5.9 & \mathrm{D}_{8} \text { symmetric } 6 \mathrm{HDM} \text { potential } & 38\end{array}$

$5.10 \mathrm{~A}_{4}$ symmetric 6HDM potential $\quad 38$

$\begin{array}{ll}5.11 \mathrm{~S}_{4} \text { symmetric } 6 \mathrm{HDM} \text { potential } & 39\end{array}$

$5.12 \Delta(54) / \mathrm{Z}_{3}$ symmetric $6 \mathrm{HDM}$ potential $\quad 40$

$5.13 \Sigma(36)$ symmetric 6HDM potential 41

6 Conclusion $\quad 42$

$\begin{array}{ll}\text { A Finding all realisable symmetries } & 43\end{array}$

A.1 Abelian symmetries 43

$\begin{array}{lll}\text { A.2 Non-Abelian symmetries } & 46\end{array}$ 
$\begin{array}{ll}\text { C The geometric minimisation method } & 48\end{array}$

$\begin{array}{lll}\text { C.1 A4-symmetric potential } & 48\end{array}$

$\begin{array}{lll}\text { C.2 } & \mathrm{S}_{4} \text {-symmetric potential } & 49\end{array}$

C.3 $\Delta(54) / Z_{3}$-symmetric potential $\quad 50$

C.4 $\Sigma(36)$-symmetric potential 51

\section{Introduction}

The discovery of a Higgs boson by the Large Hadron Collider (LHC) [1, 2], whose properties are consistent with those predicted by the Standard Model (SM), indicates that at least one Higgs doublet must be responsible for electroweak symmetry breaking (EWSB). However, there is no special reason why there should be only one Higgs doublet in Nature, and it is entirely possible that there could be additional Higgs doublets, accompanied by further Higgs bosons which could be discovered in the next run of the LHC.

The simplest example of $N$-Higgs-doublet models (NHDMs) is the class of two-Higgsdoublet models $(2 \mathrm{HDMs}),{ }^{1}$ a special example being that predicted by the minimal supersymmetric standard model (MSSM). ${ }^{2}$ Of course, the general class of 2 HDMs is much richer than the MSSM example, and indeed all possible types of 2HDMs have been well studied in the literature. ${ }^{3}$ However, 2 HDMs generally face severe phenomenological problems with flavour changing neutral currents (FCNCs) and possible charge breaking vacua, and it is common to consider restricted classes of models controlled by various symmetries.

The introduction of symmetries into $2 \mathrm{HDMs}$ provides a welcome restriction to the rather unwieldy general Higgs potential, as well as solutions to the phenomenological challenges mentioned above. For example, the remaining symmetry of the potential after EWSB can have the effect of stabilising the lightest Higgs boson, which can become a possible Dark Matter (DM) candidate. In $2 \mathrm{HDMs}$, the full list of possible symmetries of the potential is now known [6-9]: the symmetry group can be $Z_{2},\left(Z_{2}\right)^{2},\left(Z_{2}\right)^{3}, O(2), O(2) \times Z_{2}$, or $O(3) .{ }^{4}$ In $2 \mathrm{HDMs}$ these symmetries can be conserved or spontaneously violated after the EWSB, depending on the coefficients of the potential.

Generalising these results to NHDMs is technically difficult, although there has been some recent progress in this direction [12-15]. For example, with more than two doublets, there exist symmetries which are always spontaneously violated after EWSB, dubbed "frustrated symmetries" in analogy with a similar phenomenon in condensed-matter physics [16]. The idea of stabilising the lightest scalar via a preserved $Z_{p}$ symmetry (where $p$ is an integer) has also been put forward for NHDMs [17].

\footnotetext{
${ }^{1}$ For a review and references see e.g. [3].

${ }^{2}$ For a review and references see e.g. [4].

${ }^{3}$ For a recent study with an extensive list of references see e.g. [5].

${ }^{4}$ The maximum number of distinct symmetries in 2 HDMs is 13 if the custodial symmetries are included $[10,11]$. In this paper we shall not consider custodial symmetries in 3HDM.
} 
The case of three-Higgs-doublet models (3HDMs) is particularly promising for several reasons. To begin with, it is the next simplest example beyond $2 \mathrm{HDMs}$, which has been exhaustively studied in the literature. Furthermore, 3HDMs are more tractable than NHDMs, and all possible finite symmetries (but not all continuous ones) have been identified [18]. Finally, and perhaps most intriguingly, 3HDMs may shed light on the flavour problem, namely the problem of the origin and nature of the three families of quarks and leptons, including neutrinos, and their pattern of masses, mixings and $\mathrm{CP}$ violation. It is possible that the three families of quarks and leptons could be described by the same symmetries that describe the three Higgs doublets. ${ }^{5}$ In such models this family symmetry could be spontaneously broken along with the electroweak symmetry, although some remnant subgroup could survive, thereby stabilising a possible scalar DM candidate. For certain symmetries it is possible to find a vacuum expectation value (VEV) alignment that respects the original symmetry of the potential which will then be responsible for the stabilization of the DM candidate as in [17].

Despite the motivations above, $3 \mathrm{HDM}$ remain rather poorly understood, at least when compared to $2 \mathrm{HDMs}$, as can be clearly seen by the list of outstanding problems stated in [18]. The outstanding problems include [18]: the completion of the classification of possible symmetries to include continuous symmetry groups; the possible symmetry breaking patterns of the vacuum state for each choice of symmetry group; additional symmetries of the potential which are not symmetries of the kinetic terms. We would add to this list: the possible different vacuum alignments for each choice of symmetry group; the calculation of the Higgs boson mass spectrum for each such vacuum alignment; the possible DM candidates in the case where there is a preserved symmetry; the application of 3HDMs to quark and lepton flavour models [19]; the extension of 3HDMs to the case of supersymmetric (SUSY) models which motivates having three up-type Higgs doublets and three down-type Higgs doublets (six doublets in total). Finally the possible generalised CP symmetries and their breaking patterns have not yet been thoroughly studied.

The purpose of the present paper is to consider some of the aforementioned aspects of 3HDMs. We shall complete the classification of 3HDMs in terms of all possible Abelian symmetries (continuous and discrete) and all possible discrete non-Abelian symmetries. We analyse the potential in each case, and derive the conditions under which the vacuum alignments $(0,0, v),(0, v, v)$ and $(v, v, v)$ are minima of the potential. For the alignment $(0,0, v)$, which is of particular interest because of its relevance for dark matter models and the absence of FCNCs, we calculate the corresponding physical Higgs boson mass spectrum. This will lead to phenomenological constraints on the parameters in the potential, and for certain parameter choices it is possible that there could be additional light Higgs bosons which may have evaded detection at LEP. It is possible that the $125 \mathrm{GeV}$ Higgs boson could decay into these lighter Higgs bosons, providing striking new signatures for Higgs decays at the LHC. Motivated by SUSY, we then extend also the analysis to the case of three up-type Higgs doublets and three down-type Higgs doublets (six doublets in total) for the case of low ratio of VEVs $\tan \beta=v_{u} / v_{d}$. The results could be applicable to various SUSY models

\footnotetext{
${ }^{5}$ See e.g. [19].
} 
with three up-type and down-type Higgs families including MSSM where both Higgs types transform as triplets of $A_{4}[20]$ and a version of the $\mathrm{E}_{6} \mathrm{SSM}$ [21, 22] where both Higgs types transform as triplets of $\Delta(27)$ [23]. In both these examples, only the Higgs fields of the third family are (predominantly) assumed to develop VEVs which motivates the vacuum alignment $(0,0, v)$. Many of the results are also applicable to flavon models where the three Higgs doublets are replaced by three electroweak singlets.

The layout of the remainder of the paper is as follows. In section 2 we discuss the possible symmetries and symmetry breaking patterns in 3HDMs, giving the most general invariant potential, outlining the method we use for finding the local minima of the potential, and motivating the relevance of the particular alignment $(0,0, v)$ for DM. In section 3 we systematically discuss the $3 \mathrm{HDM}$ potentials which respect Abelian symmetries, deriving the conditions under which the vacuum alignments $(0,0, v),(0, v, v)$ and $(v, v, v)$ are minima of the potential and, for the alignment $(0,0, v)$, calculating the corresponding physical Higgs boson mass spectrum. In section 4 we perform an analogous analysis for the 3HDM potentials which respect non-Abelian finite symmetries. In section 5 we discuss our method for extending the results to the case of six-Higgs-doublet models (6HDMs), relevant for supersymmetric models, where the method is exemplified for $\tan \beta=1$. Section 6 concludes the paper. We also include two appendices where we review how highly symmetric potentials can be treated using orbit spaces and geometric minimisation, which are powerful techniques enabling statements to be made about the global minimum of the potential.

\section{Symmetries and symmetry breaking in 3HDMs}

When studying the symmetries of the potential, one focuses on the reparametrisation transformations, which mix the three different Higgs doublets $\phi_{a}$, where $a=1, \cdots 3$. These transformations keep the kinetic term invariant, and are either unitary (Higgs-family transformation) or anti-unitary (generalised CP-transformation), however we shall only consider the former here.

The kinetic terms of 3HDMs are invariant under the unitary transformations

$$
U: \quad \phi_{a} \mapsto U_{a b} \phi_{b}
$$

with a $3 \times 3$ unitary matrix $U_{a b}$, where $a, b=1, \cdots 3$. These transformations form the group $\mathrm{U}(3)$. However, the overall phase factor multiplication is already taken into account by the $\mathrm{U}(1)_{Y}$ from the gauge group. The kinetic terms of $3 \mathrm{HDM}$ s are therefore invariant under the $\mathrm{SU}(3)$ group of reparametrisation transformations. The group $\mathrm{SU}(3)$ has a nontrivial center $Z(\mathrm{SU}(3))=\mathbb{Z}_{3}$ generated by the diagonal matrix $\exp (2 \pi i / 3) \cdot 1_{3}$, where $1_{3}$ is the identity matrix. Therefore, the group of physically distinct unitary reparametrisation transformations respected by the kinetic terms $G_{0}$ is

$$
G_{0}=\operatorname{PSU}(3) \simeq \mathrm{SU}(3) / \mathbb{Z}_{3}
$$

In general, the Higgs potential $V$ in 3 HDMs will respect a symmetry $G$ which is some subgroup of $G_{0}$. If a symmetry $G$ is imposed on the potential $V$, it sometimes happens that 
it accidentally respects a larger symmetry. When a potential is symmetric under a group $G$ and not under any larger group containing $G$, then the group $G$ is called a realisable group [12]. A non-realisable group imposed on a potential, on the other hand, leads to a larger symmetry group of the potential. Clearly, the true symmetry properties of the potentials are reflected in realisable groups.

The full list of possible realisable symmetries $G$ of the scalar potential $V$ of 3HDMs have been found $[12,18]$. For the reader's convenience, we briefly review the methodology applied to obtain these symmetries in appendix A. Here we only list all such symmetry group which consist of the continuous Abelian symmetry groups,

$$
\mathrm{U}(1), \quad \mathrm{U}(1) \times \mathrm{U}(1), \quad \mathrm{U}(1) \times Z_{2},
$$

the finite Abelian symmetry groups,

$$
Z_{2}, \quad Z_{3}, \quad Z_{4}, \quad Z_{2} \times Z_{2},
$$

and the finite non-Abelian symmetry groups,

$$
\begin{aligned}
& D_{6}, \quad D_{8}, \quad T \simeq A_{4}, \\
& O \simeq S_{4} \\
& \left(Z_{3} \times Z_{3}\right) \rtimes Z_{2} \simeq \Delta(54) / Z_{3}, \quad\left(Z_{3} \times Z_{3}\right) \rtimes Z_{4} \simeq \Sigma(36) \text {. }
\end{aligned}
$$

A scalar $3 \mathrm{HDM}$ potential symmetric under a group $G$ can be written as

$$
V=V_{0}+V_{G}
$$

where $V_{0}$ is invariant under any phase rotation and $V_{G}$ is a collection of extra terms ensuring the symmetry group $G$. The most general invariant part of the potential has the following form

$$
V_{0}=\sum_{i}^{3}\left[-\left|\mu_{i}^{2}\right|\left(\phi_{i}^{\dagger} \phi_{i}\right)+\lambda_{i i}\left(\phi_{i}^{\dagger} \phi_{i}\right)^{2}\right]+\sum_{i j}^{3}\left[\lambda_{i j}\left(\phi_{i}^{\dagger} \phi_{i}\right)\left(\phi_{j}^{\dagger} \phi_{j}\right)+\lambda_{i j}^{\prime}\left(\phi_{i}^{\dagger} \phi_{j}\right)\left(\phi_{j}^{\dagger} \phi_{i}\right)\right] .
$$

For this potential to have a stable vacuum (bounded from below) the following conditions are required:

$$
\lambda_{i i}>0, \quad \lambda_{i j}+\lambda_{i j}^{\prime}>-2 \sqrt{\lambda_{i i} \lambda_{j j}}, \quad i \neq j=1,2,3 .
$$

The potential $V_{0}$ and associated stability conditions above are common to all the cases, Abelian and non-Abelian, which only differ by $V_{G}$.

After constructing a potential symmetric under a realisable group, we shall find the minima of the potential by explicit calculation in each case by:

- Parametrising the VEVs by $v_{i}$

- Expanding the potential around the minimum point and calculating $V\left(v_{i}\right)$

- Setting all $\partial V / \partial v_{i}$ to zero and solving these equations for $v_{i}$

- Constructing the Hessian from $\partial^{2} V / \partial v_{i} \partial v_{j}$ and requiring it to be positive definite 
We shall follow this standard method for minimising potentials symmetric under all groups listed in (2.3), (2.4) and (2.5).

In this paper we shall present the $3 \mathrm{HDM}$ potentials symmetric under each of these groups, examine the conditions for stability of the three possible minima in each case,

$$
(0,0, v), \quad(0, v, v), \quad(v, v, v)
$$

We shall focus on one of these minima, $(0,0, v)$ and study the mass spectrum around this point. The vacuum $(0,0, v)$ may either respect or breaks the original symmetry of the potential.

The motivation for considering the vacuum alignment $(0,0, v)$ is that such models can be interpreted as DM models in which the weakly interacting massive particle (WIMP) is identified as the lightest neutral scalar boson. If we define the three Higgs doublets as

$$
\phi_{1}=\left(\begin{array}{c}
H_{1}^{+} \\
\frac{H_{1}^{0}+i A_{1}^{0}}{\sqrt{2}}
\end{array}\right), \quad \phi_{2}=\left(\begin{array}{c}
H_{2}^{+} \\
\frac{H_{2}^{0}+i A_{2}^{0}}{\sqrt{2}}
\end{array}\right), \quad \phi_{3}=\left(\begin{array}{c}
H_{3}^{+} \\
\frac{v+H_{3}^{0}+i A_{3}^{0}}{\sqrt{2}}
\end{array}\right)
$$

then the vacuum $(0,0, v)$ corresponds to having two inert doublets $\left(\phi_{1}\right.$ and $\left.\phi_{2}\right)$ and one active doublet $\left(\phi_{3}\right)$. "Inert" means not only zero VEV but also no couplings to fermions. To be precise, if the symmetry of the potential after EWSB is $G$, we assign a quantum number to each doublet according to the generator of $G$. To make sure that the entire Lagrangian and not only the scalar potential is $G$ symmetric, we set the $G$ quantum number of all SM particles equal to the $G$ quantum number of the only doublet that couples to them i.e. the active doublet $\phi_{3}$. With this charge assignment FCNCs are avoided as the extra doublets are forbidden to couple to fermions by $G$ conservation. In each case, with the vacuum alignment $(0,0, v)$, the CP-even/odd neutral fields resulting from the inert doublets $\left(H_{1}^{0}, H_{2}^{0}, A_{1}^{0}, A_{2}^{0}\right)$ could in principle be dark matter (DM) candidates since only the active doublet acquires a VEV and couples to the fermions. To stabilize the DM candidate from decaying into SM particles, we make use of the remnant symmetry of the potential after EWSB.

For the special cases of the non-Abelian symmetries listed in eq. (2.5), when the symmetry of the potential is sufficiently large, a powerful geometric method for minimising the potential has been introduced [24]. From this list of finite non-Abelian symmetries in (2.5), the following symmetries are "frustrated" 6 since they are inevitably broken after EWSB:

$$
A_{4}, \quad S_{4}, \quad \Delta(54) / Z_{3}, \quad \Sigma(36) .
$$

For each of these four cases we rewrite the potential in terms of the bilinears and using the geometric method introduced in [24] find all minima of the potential. The results of this

\footnotetext{
${ }^{6}$ In the case of $2 \mathrm{HDMs}$, it is always possible to find a vacuum alignment which respects the symmetry of the potential. However, in NHDMs with $N>2$ when the potential is highly symmetric (having $M_{i}=0$ when written in terms of the bilinears as in eq. (B.3)) any vacuum alignment breaks the symmetry of the potential. Therefore, these symmetry groups can never be conserved after EWSB. The origin of this phenomena and some 3HDM examples were discussed in [16]. These symmetries are not specific to doublets. They can arise when the representation of the electroweak group has lower dimensionality than the horizontal (Higgs family) space, i.e. more than one singlet, more than two doublets, more than three triplets, etc.
} 


\begin{tabular}{|c|c|c|}
\hline Symmetry & Diagonal Generators & Potential \\
\hline $\mathrm{U}(1) \times \mathrm{U}(1)$ & $\left(e^{-i \alpha}, e^{i \alpha}, 1\right), \quad\left(e^{-2 i \beta / 3}, e^{i \beta / 3}, e^{i \beta / 3}\right)$ & $V_{0}$ \\
\hline $\mathrm{U}(1)$ & $\left(e^{-i \alpha}, e^{i \alpha}, 1\right)$ & $V_{0}+\lambda_{1}\left(\phi_{1}^{\dagger} \phi_{3}\right)\left(\phi_{2}^{\dagger} \phi_{3}\right)+h . c$. \\
\hline $\mathrm{U}(1) \times Z_{2}$ & $\left(e^{-2 i \beta / 3}, e^{i \beta / 3}, e^{i \beta / 3}\right), \quad(-1,-1,1)$ & $V_{0}+\lambda_{1}\left(\phi_{2}^{\dagger} \phi_{3}\right)^{2}+h . c$. \\
\hline$Z_{2}$ & $(-1,-1,1)$ & $\begin{array}{l}V_{0}-\mu_{12}^{\prime 2}\left(\phi_{1}^{\dagger} \phi_{2}\right)+\lambda_{1}\left(\phi_{1}^{\dagger} \phi_{2}\right)^{2}+\lambda_{2}\left(\phi_{2}^{\dagger} \phi_{3}\right)^{2}+ \\
\lambda_{3}\left(\phi_{3}^{\dagger} \phi_{1}\right)^{2}+\text { h.c. }\end{array}$ \\
\hline$Z_{3}$ & $\left(\omega, \omega^{2}, 1\right)$ & $\begin{array}{l}V_{0}+\lambda_{1}\left(\phi_{1}^{\dagger} \phi_{2}\right)\left(\phi_{3}^{\dagger} \phi_{2}\right)+\lambda_{2}\left(\phi_{2}^{\dagger} \phi_{3}\right)\left(\phi_{1}^{\dagger} \phi_{3}\right)+ \\
\lambda_{3}\left(\phi_{3}^{\dagger} \phi_{1}\right)\left(\phi_{2}^{\dagger} \phi_{1}\right)+\text { h.c. }\end{array}$ \\
\hline$Z_{4}$ & $(i,-i, 1)$ & $V_{0}+\lambda_{1}\left(\phi_{3}^{\dagger} \phi_{1}\right)\left(\phi_{3}^{\dagger} \phi_{2}\right)+\lambda_{2}\left(\phi_{1}^{\dagger} \phi_{2}\right)^{2}+h . c$. \\
\hline$Z_{2} \times Z_{2}$ & $(-1,1,1), \quad(1,-1,1)$ & $V_{0}+\lambda_{1}\left(\phi_{1}^{\dagger} \phi_{2}\right)^{2}+\lambda_{2}\left(\phi_{2}^{\dagger} \phi_{3}\right)^{2}+\lambda_{3}\left(\phi_{3}^{\dagger} \phi_{1}\right)^{2}+$ h.c. \\
\hline
\end{tabular}

Table 1. All Abelian symmetries realisable in the scalar sector of 3 HDMs. $V_{0}$ is the phase invariant part of the potential presented in eq. (3.2).

method are presented in appendix $\mathrm{C}$ using the orbit space formalism briefly reviewed in appendix B.

\section{Analysis of Abelian 3HDMs}

In this section we study all Abelian symmetries in 3HDM potentials. Table 1 lists all these symmetry groups, their generators and their corresponding potentials.

\section{$3.1 \quad \mathrm{U}(1) \times \mathrm{U}(1)$ symmetric $3 \mathrm{HDM}$ potential}

The $\mathrm{U}(1) \times \mathrm{U}(1) \subset \mathrm{PSU}(3)$ group is generated by

$$
U_{1}(1)=\left(\begin{array}{ccc}
e^{-i \alpha} & 0 & 0 \\
0 & e^{i \alpha} & 0 \\
0 & 0 & 1
\end{array}\right), \quad U_{2}(1)=\left(\begin{array}{ccc}
e^{-2 i \beta / 3} & 0 & 0 \\
0 & e^{i \beta / 3} & 0 \\
0 & 0 & e^{i \beta / 3}
\end{array}\right)
$$

where $\alpha, \beta \in[0,2 \pi)$.

The most general $\mathrm{U}(1) \times \mathrm{U}(1)$-symmetric $3 \mathrm{HDM}$ potential has the following form:

$$
\begin{aligned}
V_{0}= & -\mu_{1}^{2}\left(\phi_{1}^{\dagger} \phi_{1}\right)-\mu_{2}^{2}\left(\phi_{2}^{\dagger} \phi_{2}\right)-\mu_{3}^{2}\left(\phi_{3}^{\dagger} \phi_{3}\right) \\
& +\lambda_{11}\left(\phi_{1}^{\dagger} \phi_{1}\right)^{2}+\lambda_{22}\left(\phi_{2}^{\dagger} \phi_{2}\right)^{2}+\lambda_{33}\left(\phi_{3}^{\dagger} \phi_{3}\right)^{2} \\
& +\lambda_{12}\left(\phi_{1}^{\dagger} \phi_{1}\right)\left(\phi_{2}^{\dagger} \phi_{2}\right)+\lambda_{23}\left(\phi_{2}^{\dagger} \phi_{2}\right)\left(\phi_{3}^{\dagger} \phi_{3}\right)+\lambda_{31}\left(\phi_{3}^{\dagger} \phi_{3}\right)\left(\phi_{1}^{\dagger} \phi_{1}\right) \\
& +\lambda_{12}^{\prime}\left(\phi_{1}^{\dagger} \phi_{2}\right)\left(\phi_{2}^{\dagger} \phi_{1}\right)+\lambda_{23}^{\prime}\left(\phi_{2}^{\dagger} \phi_{3}\right)\left(\phi_{3}^{\dagger} \phi_{2}\right)+\lambda_{31}^{\prime}\left(\phi_{3}^{\dagger} \phi_{1}\right)\left(\phi_{1}^{\dagger} \phi_{3}\right)
\end{aligned}
$$

which is symmetric under any phase rotation of doublets.

We find all possible extrema of the potential by requiring

$$
\left(\frac{\partial V}{\partial \phi_{i}}\right)_{\phi_{i}=\left\langle\phi_{i}\right\rangle}=0, \quad\left(\frac{\partial V}{\partial \phi_{i}^{\dagger}}\right)_{\phi_{i}=\left\langle\phi_{i}\right\rangle}=0, \quad i=1,2,3
$$


which results in the following solutions

$$
(0,0, v), \quad(0, v, v), \quad(v, v, v)
$$

where in each case permutations are allowed and in general doublets could acquire non-equal VEVs $\left(v_{1} \neq v_{2} \neq v_{3}\right)$. In the following sections, however, the conditions are derived for the presented VEV alignment and the results do not apply to permuted VEV alignments.

To find the conditions on the parameters which are required for the above points to be minima of the potential, we construct the Hessian

$$
\left|\begin{array}{ccc}
\frac{\partial^{2} V}{\partial \phi_{1} \partial \phi_{1}^{\dagger}} & \frac{\partial^{2} V}{\partial \phi_{1} \partial \phi_{2}^{\dagger}} & \frac{\partial^{2} V}{\partial \phi_{1} \partial \phi_{3}^{\dagger}} \\
\frac{\partial^{2} V}{\partial \phi_{2} \partial \phi_{1}^{\dagger}} & \frac{\partial^{2} V}{\partial \phi_{2} \partial \phi_{2}^{\dagger}} & \frac{\partial^{2} V}{\partial \phi_{2} \partial \phi_{3}^{\dagger}} \\
\frac{\partial^{2} V}{\partial \phi_{3} \partial \phi_{1}^{\dagger}} & \frac{\partial^{2} V}{\partial \phi_{3} \partial \phi_{2}^{\dagger}} & \frac{\partial^{2} V}{\partial \phi_{3} \partial \phi_{3}^{\dagger}}
\end{array}\right|>0
$$

and require it to be positive definite (see e.g. [25]).

We find the following results:

1. Point $\left(0,0, \frac{v}{\sqrt{2}}\right)$ breaks the symmetry of the potential to $U_{1}(1)$ and becomes the minimum at

$$
v^{2}=\frac{\mu_{3}^{2}}{\lambda_{33}}
$$

provided the following conditions are satisfied:

$$
\begin{array}{ll}
\text { - } & -\mu_{1}^{2}+\frac{1}{2}\left(\lambda_{31}+\lambda_{31}^{\prime}\right) v^{2}>0 \\
\text { - } & -\mu_{2}^{2}+\frac{1}{2}\left(\lambda_{23}+\lambda_{23}^{\prime}\right) v^{2}>0 \\
\text { - } & \mu_{3}^{2}>0
\end{array}
$$

However, all these conditions are already required for the positivity of mass eigenstates at point $\left(0,0, \frac{v}{\sqrt{2}}\right)$. Therefore, we conclude that this point is always a minimum of the potential.

2. Point $\left(0, \frac{v}{\sqrt{2}}, \frac{v}{\sqrt{2}}\right)$ breaks the symmetry of the potential to a $Z_{2}$ generated by $(1,-1,-1)$. This point becomes the minimum of the potential at

$$
v^{2}=\frac{2 \mu_{3}^{2}}{2 \lambda_{33}+\lambda_{23}+\lambda_{23}^{\prime}}=\frac{2 \mu_{2}^{2}}{2 \lambda_{22}+\lambda_{23}+\lambda_{23}^{\prime}}
$$

when the following conditions are satisfied:

- $\lambda_{22}>0$

- $\lambda_{33}>0$

- $\quad-2 \mu_{1}^{2}+\left(\lambda_{12}+\lambda_{12}^{\prime}+\lambda_{31}+\lambda_{31}^{\prime}\right) v^{2}>0$

- $4 \lambda_{22} \lambda_{33}>\left(\lambda_{23}+\lambda_{23}^{\prime}\right)^{2}$ 
3. Point $\left(\frac{v}{\sqrt{2}}, \frac{v}{\sqrt{2}}, \frac{v}{\sqrt{2}}\right)$ breaks the symmetry of the potential completely and becomes the minimum at

$$
\begin{aligned}
v^{2} & =\frac{2 \mu_{3}^{2}}{2 \lambda_{33}+\lambda_{23}+\lambda_{23}^{\prime}+\lambda_{31}+\lambda_{31}^{\prime}} \\
& =\frac{2 \mu_{2}^{2}}{2 \lambda_{22}+\lambda_{12}+\lambda_{12}^{\prime}+\lambda_{23}+\lambda_{23}^{\prime}} \\
& =\frac{2 \mu_{1}^{2}}{2 \lambda_{11}+\lambda_{12}+\lambda_{12}^{\prime}+\lambda_{31}+\lambda_{31}^{\prime}}
\end{aligned}
$$

with the following conditions:

- $4 \lambda_{11} \lambda_{22}>\left(\lambda_{12}+\lambda_{12}^{\prime}\right)^{2}$

- $4 \lambda_{11} \lambda_{22} \lambda_{33}+\left(\lambda_{12}+\lambda_{12}^{\prime}\right)\left(\lambda_{31}+\lambda_{32}^{\prime}\right)\left(\lambda_{23}+\lambda_{23}^{\prime}\right)>$

$$
\lambda_{11}\left(\lambda_{23}+\lambda_{23}^{\prime}\right)^{2}+\lambda_{22}\left(\lambda_{31}+\lambda_{31}^{\prime}\right)^{2}+\lambda_{33}\left(\lambda_{12}+\lambda_{12}^{\prime}\right)^{2}
$$

Higgs mass spectrum for $\left(0,0, \frac{v}{\sqrt{2}}\right)$

The VEV alignment $\left(0,0, \frac{v}{\sqrt{2}}\right)$ breaks the $\mathrm{U}(1) \times \mathrm{U}(1)$ symmetry of the potential to $U_{1}(1)$ where the fields from the first and the second doublets are assigned $U_{1}(1)$ quantum numbers -1 and 1 respectively, and the fields from the third doublet get $U_{1}(1)$ quantum number zero. We can write the generator as:

$$
U_{1}(1)=\alpha(-1,1,0) .
$$

Writing the generator of the group in this form facilitates the task of assigning $U_{1}(1)$ charges to the doublets. We require all SM fields to have the same $U_{1}(1)$ charge as the active doublet i.e. zero so that the whole Lagrangian is $U_{1}(1)$-symmetric. Having defined the doublet as in (2.10) the lightest neutral field from the first and the second doublet which is stabilised by the remaining $U_{1}(1)$ symmetry is a viable DM candidate.

Expanding the potential around the vacuum point $\left(0,0, \frac{v}{\sqrt{2}}\right)$, with

$$
v^{2}=\frac{\mu_{3}^{2}}{\lambda_{33}}
$$

results in the following Higgs mass spectrum:

$$
\begin{array}{ll}
\mathbf{A}_{3}: & m^{2}=0 \\
\mathbf{H}_{3}^{ \pm}: & m^{2}=0 \\
\mathbf{H}_{3}: & m^{2}=2 \mu_{3}^{2} \\
\mathbf{H}_{2}^{ \pm}: & m^{2}=-\mu_{2}^{2}+\frac{1}{2} \lambda_{23} v^{2} \\
\mathbf{H}_{1}^{ \pm}: & m^{2}=-\mu_{1}^{2}+\frac{1}{2} \lambda_{31} v^{2} \\
\mathbf{H}_{2}: & m^{2}=-\mu_{2}^{2}+\frac{1}{2}\left(\lambda_{23}+\lambda_{23}^{\prime}\right) v^{2} \\
\mathbf{A}_{2}: & m^{2}=-\mu_{2}^{2}+\frac{1}{2}\left(\lambda_{23}+\lambda_{23}^{\prime}\right) v^{2} \\
\mathbf{H}_{1}: & m^{2}=-\mu_{1}^{2}+\frac{1}{2}\left(\lambda_{31}+\lambda_{31}^{\prime}\right) v^{2} \\
\mathbf{A}_{1}: & m^{2}=-\mu_{1}^{2}+\frac{1}{2}\left(\lambda_{31}+\lambda_{31}^{\prime}\right) v^{2}
\end{array}
$$


where the fields appearing in the doublets in (2.10) are the same as the mass eigenstates (shown in bold text).

Note that the fields from the third doublet play the role of the SM-Higgs doublet fields, namely a massless neutral Goldstone boson $\left(\mathbf{A}_{3}\right)$, two massless charged Goldstone bosons $\left(\mathbf{H}_{3}^{ \pm}\right)$and the SM-Higgs boson $\left(\mathbf{H}_{3}\right)$.

Positivity of the mass eigenstates enforces the following extra conditions on the parameters of the potential:

$$
\begin{array}{ll}
\text { - } & \lambda_{33}>0 \\
\text { - } & \mu_{3}^{2}>0 \\
\text { - } & 2 \mu_{1}^{2}<\lambda_{31} v^{2} \\
\text { - } & 2 \mu_{1}^{2}<\left(\lambda_{31}+\lambda^{\prime}{ }_{31}\right) v^{2} \\
\text { - } & 2 \mu_{2}^{2}<\lambda_{23} v^{2} \\
\text { - } & 2 \mu_{2}^{2}<\left(\lambda_{23}+\lambda^{\prime}{ }_{23}\right) v^{2}
\end{array}
$$

\section{$3.2 \mathrm{U}(1)$ symmetric $3 \mathrm{HDM}$ potential}

A potential symmetric under the $U_{1}(1)$ group in (3.9) contains the following terms: ${ }^{7}$

$$
V_{U_{1}(1)}=\lambda_{1}\left(\phi_{1}^{\dagger} \phi_{3}\right)\left(\phi_{2}^{\dagger} \phi_{3}\right)+\text { h.c. }
$$

in addition to $V_{0}$ in eq. (3.2).

The possible minima of this potential are:

1. Point $\left(0,0, \frac{v}{\sqrt{2}}\right)$ respects the symmetry of the potential and becomes the minimum at

$$
v^{2}=\frac{\mu_{3}^{2}}{\lambda_{33}}
$$

provided the following conditions are satisfied:

$$
\begin{array}{ll}
\text { - } & -\mu_{1}^{2}+\frac{1}{2}\left(\lambda_{31}+\lambda_{31}^{\prime}\right) v^{2}>0 \\
\text { - } & -\mu_{2}^{2}+\frac{1}{2}\left(\lambda_{23}+\lambda_{23}^{\prime}\right) v^{2}>0 \\
\text { - } & \mu_{3}^{2}>0
\end{array}
$$

where the third condition is already required for the positivity of mass eigenstates at point $\left(0,0, \frac{v}{\sqrt{2}}\right)$.

2. Point $\left(0, \frac{v}{\sqrt{2}}, \frac{v}{\sqrt{2}}\right)$ only becomes the minimum if $\lambda_{1}=0$ which reduces the symmetry of the potential to $\mathrm{U}(1) \times \mathrm{U}(1)$. We conclude that this point is not a minimum of the $\mathrm{U}(1)$-symmetric potential.

\footnotetext{
${ }^{7}$ A general $U_{2}(1)$ symmetric $3 \mathrm{HDM}$ potential contains:$$
V_{U_{2}(1)}=-\mu_{23}^{2}\left(\phi_{2}^{\dagger} \phi_{3}\right)+\lambda_{1}\left(\phi_{1}^{\dagger} \phi_{1}\right)\left(\phi_{2}^{\dagger} \phi_{3}\right)+\lambda_{2}\left(\phi_{2}^{\dagger} \phi_{2}\right)\left(\phi_{2}^{\dagger} \phi_{3}\right)
$$$$
+\lambda_{3}\left(\phi_{3}^{\dagger} \phi_{3}\right)\left(\phi_{2}^{\dagger} \phi_{3}\right)+\lambda_{4}\left(\phi_{2}^{\dagger} \phi_{3}\right)^{2}+\lambda_{5}\left(\phi_{2}^{\dagger} \phi_{1}\right)\left(\phi_{1}^{\dagger} \phi_{3}\right)+\text { h.c. }
$$ 
3. Point $\left(\frac{v}{\sqrt{2}}, \frac{v}{\sqrt{2}}, \frac{v}{\sqrt{2}}\right)$ breaks the symmetry of the potential completely and becomes the minimum at

$$
\begin{aligned}
v^{2} & =\frac{2 \mu_{3}^{2}}{2 \lambda_{33}+\lambda_{23}+\lambda_{23}^{\prime}+\lambda_{31}+\lambda_{31}^{\prime}+2 \lambda_{1}} \\
& =\frac{2 \mu_{2}^{2}}{2 \lambda_{22}+\lambda_{12}+\lambda_{12}^{\prime}+\lambda_{23}+\lambda_{23}^{\prime}+\lambda_{1}} \\
& =\frac{2 \mu_{1}^{2}}{2 \lambda_{11}+\lambda_{12}+\lambda_{12}^{\prime}+\lambda_{31}+\lambda_{31}^{\prime}+\lambda_{1}}
\end{aligned}
$$

with the following conditions:

- $2 \lambda_{11}>\lambda_{1}$

- $2 \lambda_{22}>\lambda_{1}$

- $\lambda_{33}>\lambda_{1}$

- $\left(\lambda_{1}-2 \lambda_{11}\right)\left(\lambda_{1}-2 \lambda_{22}\right)>\left(\lambda_{12}+\lambda_{12}^{\prime}\right)^{2}$

- $2\left(\lambda_{12}+\lambda_{12}^{\prime}\right)\left(\lambda_{31}+\lambda_{31}^{\prime}+2 \lambda_{1}\right)\left(\lambda_{23}+\lambda_{23}^{\prime}+2 \lambda_{1}\right)$

$+\left(2 \lambda_{11}-\lambda_{1}\right)\left(2 \lambda_{22}-\lambda_{1}\right)\left(2 \lambda_{33}-2 \lambda_{1}\right)>\left(2 \lambda_{33}-2 \lambda_{1}\right)\left(\lambda_{12}+\lambda_{12}^{\prime}\right)^{2}$

$+\left(2 \lambda_{11}-\lambda_{1}\right)\left(\lambda_{23}+\lambda_{23}^{\prime}+2 \lambda_{1}\right)^{2}+\left(2 \lambda_{22}-\lambda_{1}\right)\left(\lambda_{31}+\lambda_{31}^{\prime}+2 \lambda_{1}\right)^{2}$

\section{Higgs mass spectrum for $\left(0,0, \frac{v}{\sqrt{2}}\right)$}

Defining the doublets similarly to the previous case and expanding the potential around the vacuum point $\left(0,0, \frac{v}{\sqrt{2}}\right)$, with

$$
v^{2}=\frac{\mu_{3}^{2}}{\lambda_{33}},
$$

results in a mass spectrum of the following form:

$$
\begin{array}{ll}
\mathbf{A}_{3}: & m^{2}=0 \\
\mathbf{H}_{3}^{ \pm}: & m^{2}=0 \\
\mathbf{H}_{3}: & m^{2}=2 \mu_{3}^{2} \\
\mathbf{H}_{2}^{ \pm}: & m^{2}=-\mu_{2}^{2}+\frac{1}{2} \lambda_{23} v^{2} \\
\mathbf{H}_{1}^{ \pm}: & m^{2}=-\mu_{1}^{2}+\frac{1}{2} \lambda_{31} v^{2} \\
\mathbf{H}_{2} \equiv \frac{a H_{2}^{0}+H_{1}^{0}}{\sqrt{1+a^{2}}}: \quad m^{2}=\frac{1}{2}(X-\sqrt{Y}) \\
\mathbf{H}_{1} \equiv \frac{b H_{2}^{0}+H_{1}^{0}}{\sqrt{1+b^{2}}}: \quad m^{2}=\frac{1}{2}(X+\sqrt{Y}) \\
\mathbf{A}_{2} \equiv \frac{-a A_{2}^{0}+A_{1}^{0}}{\sqrt{1+a^{2}}}: \quad m^{2}=\frac{1}{2}(X-\sqrt{Y}) \\
\mathbf{A}_{1} \equiv \frac{-b A_{2}^{0}+A_{1}^{0}}{\sqrt{1+b^{2}}:} \quad m^{2}=\frac{1}{2}(X+\sqrt{Y}) \\
\text { where } \quad X=-\mu_{1}^{2}-\mu_{2}^{2}+\frac{1}{2}\left(\lambda_{23}+\lambda_{31}+\lambda_{23}^{\prime}+\lambda_{31}^{\prime}\right) v^{2} \\
\quad Y=\left(\lambda_{1} v^{2}\right)^{2}+\left[\mu_{1}^{2}-\mu_{2}^{2}+\frac{1}{2}\left(\lambda_{23}-\lambda_{31}+\lambda_{23}^{\prime}-\lambda_{31}^{\prime}\right) v^{2}\right]^{2}
\end{array}
$$




$$
\begin{aligned}
& a=\frac{1}{\lambda_{1} v^{2}}\left[\mu_{1}^{2}-\mu_{2}^{2}+\frac{1}{2}\left(\lambda_{23}-\lambda_{31}+\lambda_{23}^{\prime}-\lambda_{31}^{\prime}\right) v^{2}-\sqrt{Y}\right] \\
& b=\frac{1}{\lambda_{1} v^{2}}\left[\mu_{1}^{2}-\mu_{2}^{2}+\frac{1}{2}\left(\lambda_{23}-\lambda_{31}+\lambda_{23}^{\prime}-\lambda_{31}^{\prime}\right) v^{2}+\sqrt{Y}\right]
\end{aligned}
$$

The lightest neutral eigenstate among the ones from the first and the second doublet, $\mathbf{H}_{1}, \mathbf{H}_{2}$ and $\mathbf{A}_{1}, \mathbf{A}_{2}$, which is stabilised by the conserved $U_{1}(1)$ symmetry is a viable DM candidate.

\section{$3.3 \mathrm{U}(1) \times \mathrm{Z}_{2}$ symmetric $3 \mathrm{HDM}$ potential}

In addition to $V_{0}$ in eq. (3.2) the $U_{2}(1) \times Z_{2}$-symmetric $3 \mathrm{HDM}$ potential contains the following term:

$$
V_{\mathrm{U}(1) \times Z_{2}}=\lambda_{1}\left(\phi_{2}^{\dagger} \phi_{3}\right)^{2}+\text { h.c. }
$$

This term is symmetric under

$$
U_{2}(1)=\operatorname{diag}\left(e^{-2 i \beta / 3}, e^{i \beta / 3}, e^{i \beta / 3}\right), \quad \text { and } \quad Z_{2}=\operatorname{diag}(-1,-1,1) .
$$

The possible minima of this potential are:

1. Point $\left(0,0, \frac{v}{\sqrt{2}}\right)$ breaks the symmetry of the potential to $Z_{2}$ and becomes the minimum at

$$
v^{2}=\frac{\mu_{3}^{2}}{\lambda_{33}}
$$

provided the following conditions are satisfied:

$$
\begin{array}{ll}
\text { - } & -\mu_{2}^{2}+\frac{1}{2}\left(\lambda_{23}+\lambda_{23}^{\prime}\right) v^{2}>0 \\
\text { - } & -\mu_{1}^{2}+\frac{1}{2}\left(\lambda_{31}+\lambda_{31}^{\prime}\right) v^{2}>0 \\
\text { - } & \mu_{3}^{2}>0
\end{array}
$$

The last two conditions are already required for the positivity of mass eigenstates at point $\left(0,0, \frac{v}{\sqrt{2}}\right)$.

2. Point $\left(0, \frac{v}{\sqrt{2}}, \frac{v}{\sqrt{2}}\right)$ breaks the symmetry of the potential to a $Z_{2}$ generated by $(1,-1,-1)$ and becomes the minimum at

$$
v^{2}=\frac{2 \mu_{3}^{2}}{2 \lambda_{33}+\lambda_{23}+\lambda_{23}^{\prime}+\lambda_{31}+\lambda_{31}^{\prime}+2 \lambda_{1}}=\frac{2 \mu_{2}^{2}}{2 \lambda_{22}+\lambda_{12}+\lambda_{12}^{\prime}+\lambda_{23}+\lambda_{23}^{\prime}+2 \lambda_{1}}
$$

when the following conditions are satisfied:

- $\quad-2 \mu_{1}^{2}+\left(\lambda_{12}+\lambda_{12}^{\prime}+\lambda_{31}+\lambda_{31}^{\prime}\right) v^{2}>0$

- $2 \lambda_{22}-2 \lambda_{1}-\lambda_{12}-\lambda_{12}^{\prime}>0$

- $2 \lambda_{33}-2 \lambda_{1}-\lambda_{31}-\lambda_{31}^{\prime}>0$

- $\left(2 \lambda_{22}-2 \lambda_{1}-\lambda_{12}-\lambda_{12}^{\prime}\right)\left(2 \lambda_{33}-2 \lambda_{1}-\lambda_{31}-\lambda_{31}^{\prime}\right)>\left(4 \lambda_{1}+\lambda_{23}+\lambda_{23}^{\prime}\right)^{2}$ 
3. Point $\left(\frac{v}{\sqrt{2}}, \frac{v}{\sqrt{2}}, \frac{v}{\sqrt{2}}\right)$ breaks the symmetry of the potential completely and becomes the minimum at

$$
\begin{aligned}
v^{2} & =\frac{2 \mu_{3}^{2}}{2 \lambda_{33}+\lambda_{23}+\lambda_{23}^{\prime}+\lambda_{31}+\lambda_{31}^{\prime}+2 \lambda_{1}} \\
& =\frac{2 \mu_{2}^{2}}{2 \lambda_{22}+\lambda_{12}+\lambda_{12}^{\prime}+\lambda_{23}+\lambda_{23}^{\prime}+2 \lambda_{1}} \\
& =\frac{2 \mu_{1}^{2}}{2 \lambda_{11}+\lambda_{12}+\lambda_{12}^{\prime}+\lambda_{31}+\lambda_{31}^{\prime}}
\end{aligned}
$$

with the following conditions:

- $\lambda_{11}>0$

- $\lambda_{22}>\lambda_{1}$

- $\lambda_{33}>\lambda_{1}$

- $4 \lambda_{11}\left(\lambda_{22}-\lambda_{1}\right)>\left(\lambda_{12}+\lambda_{12}^{\prime}\right)^{2}$

- $\left(\lambda_{12}+\lambda_{12}^{\prime}\right)\left(\lambda_{31}+\lambda_{31}^{\prime}+8 \lambda_{1}\right)\left(\lambda_{23}+\lambda_{23}^{\prime}\right)+4 \lambda_{11}\left(\lambda_{22}-\lambda_{1}\right)\left(\lambda_{33}-\lambda_{1}\right) \quad>$

$$
\left(-\lambda_{1}+\lambda_{33}\right)\left(\lambda_{12}+\lambda_{12}^{\prime}\right)^{2}+\lambda_{11}\left(\lambda_{23}+\lambda_{23}^{\prime}+8 \lambda_{1}\right)^{2}+\left(-\lambda_{1}+\lambda_{22}\right)\left(\lambda_{31}+\lambda_{31}^{\prime}\right)^{2}
$$

\section{Higgs mass spectrum for $\left(0,0, \frac{v}{\sqrt{2}}\right)$}

Expanding the potential around the vacuum point $\left(0,0, \frac{v}{\sqrt{2}}\right)$, with

$$
v^{2}=\frac{\mu_{3}^{2}}{\lambda_{33}}
$$

the mass spectrum appears as follows:

$$
\begin{array}{ll}
\mathbf{A}_{3}: & m^{2}=0 \\
\mathbf{H}_{3}^{ \pm}: & m^{2}=0 \\
\mathbf{H}_{3}: & m^{2}=2 \mu_{3}^{2} \\
\mathbf{H}_{2}^{ \pm}: & m^{2}=-\mu_{2}^{2}+\frac{1}{2} \lambda_{23} v^{2} \\
\mathbf{H}_{1}^{ \pm}: & m^{2}=-\mu_{1}^{2}+\frac{1}{2} \lambda_{31} v^{2} \\
\mathbf{H}_{2}: & m^{2}=-\mu_{2}^{2}+\frac{1}{2}\left(\lambda_{23}+\lambda_{23}^{\prime}+2 \lambda_{1}\right) v^{2} \\
\mathbf{A}_{2}: & m^{2}=-\mu_{2}^{2}+\frac{1}{2}\left(\lambda_{23}+\lambda_{23}^{\prime}-2 \lambda_{1}\right) v^{2} \\
\mathbf{H}_{1}: & m^{2}=-\mu_{1}^{2}+\frac{1}{2}\left(\lambda_{31}+\lambda_{31}^{\prime}\right) v^{2} \\
\mathbf{A}_{1}: & m^{2}=-\mu_{1}^{2}+\frac{1}{2}\left(\lambda_{31}+\lambda_{31}^{\prime}\right) v^{2}
\end{array}
$$

The lightest neutral field from the first and the second doublet which is stabilised by the remaining $Z_{2}$ symmetry is a viable DM candidate. 


\section{$3.4 \quad \mathrm{Z}_{2}$ symmetric $3 \mathrm{HDM}$ potential}

Constructing the $Z_{2}$-symmetric part of the potential depends on the generator of the group. The $Z_{2}$ generator which forbids FCNCs has the following form

$$
a=\operatorname{diag}(-1,-1,1) \text {. }
$$

The terms ensuring the $Z_{2}$ group generated by $a$ are ${ }^{8}$

$$
V_{Z_{2}}=V_{0}-\mu_{12}^{\prime 2}\left(\phi_{1}^{\dagger} \phi_{2}\right)+\lambda_{1}\left(\phi_{1}^{\dagger} \phi_{2}\right)^{2}+\lambda_{2}\left(\phi_{2}^{\dagger} \phi_{3}\right)^{2}+\lambda_{3}\left(\phi_{3}^{\dagger} \phi_{1}\right)^{2}+\text { h.c. }
$$

which need to be added to $V_{0}$ in eq. (3.2) to result in a uniquely $Z_{2}$-symmetric 3HDM potential.

The possible minima of this potential are:

1. Point $\left(0,0, \frac{v}{\sqrt{2}}\right)$ respects the symmetry of the potential and becomes the minimum at

$$
v^{2}=\frac{\mu_{3}^{2}}{\lambda_{33}}
$$

provided the following conditions are satisfied:

$$
\begin{array}{ll}
\text { - } & -\mu_{2}^{2}+\frac{1}{2}\left(\lambda_{23}+\lambda_{23}^{\prime}\right) v^{2}>0 \\
\text { - } & -\mu_{1}^{2}+\frac{1}{2}\left(\lambda_{31}+\lambda_{31}^{\prime}\right) v^{2}>0 \\
\text { - } & \left(-\mu_{1}^{2}+\frac{1}{2}\left(\lambda_{31}+\lambda_{31}^{\prime}\right) v^{2}\right)\left(-\mu_{2}^{2}+\frac{1}{2}\left(\lambda_{23}+\lambda_{23}^{\prime}\right) v^{2}\right)>\left(\mu_{12}^{\prime 2}\right)^{2} \\
\text { - } & \mu_{3}^{2}>0
\end{array}
$$

The last condition is already required for the positivity of mass eigenstates at point $\left(0,0, \frac{v}{\sqrt{2}}\right)$.

2. Point $\left(0, \frac{v}{\sqrt{2}}, \frac{v}{\sqrt{2}}\right)$ can only be a minimum of this potential if $\mu_{12}^{\prime 2}=0$ which makes the potential $Z_{2} \times Z_{2}$-symmetric. We therefore conclude that this point is not a minimum of the $Z_{2}$-symmetric potential.

3. Point $\left(\frac{v}{\sqrt{2}}, \frac{v}{\sqrt{2}}, \frac{v}{\sqrt{2}}\right)$ breaks the symmetry of the potential completely and becomes the minimum at

$$
\begin{aligned}
v^{2} & =\frac{2 \mu_{3}^{2}}{2 \lambda_{33}+\lambda_{23}+\lambda_{23}^{\prime}+\lambda_{31}+\lambda_{31}^{\prime}+2 \lambda_{2}+2 \lambda_{3}} \\
& =\frac{2 \mu_{2}^{2}+2 \mu_{12}^{\prime 2}}{2 \lambda_{22}+\lambda_{12}+\lambda_{12}^{\prime}+\lambda_{23}+\lambda_{23}^{\prime}+2 \lambda_{1}+2 \lambda_{2}}
\end{aligned}
$$

\footnotetext{
${ }^{8}$ Note that the only symmetry group of this potential is the $Z_{2}$ group generated by $a$. However, this is not the only $Z_{2}$ symmetric potential that can be written. A potential with the following terms added to $V_{0}$ is also symmetric only under the $Z_{2}$ group generated by $a$ :

$$
V_{Z_{2}}^{\prime}=\lambda_{1}\left(\phi_{1}^{\dagger} \phi_{2}\right)^{2}+\lambda_{2}\left(\phi_{2}^{\dagger} \phi_{3}\right)^{2}+\lambda_{3}\left(\phi_{3}^{\dagger} \phi_{1}\right)^{2}+\lambda_{4}\left(\phi_{1}^{\dagger} \phi_{2}\right)\left[\left(\phi_{1}^{\dagger} \phi_{1}\right)+\left(\phi_{2}^{\dagger} \phi_{2}\right)\right]+\lambda_{5}\left(\phi_{1}^{\dagger} \phi_{3}\right)\left(\phi_{2}^{\dagger} \phi_{3}\right)+h . c .
$$
}




$$
=\frac{2 \mu_{1}^{2}+2 \mu_{12}^{\prime 2}}{2 \lambda_{11}+\lambda_{12}+\lambda_{12}^{\prime}+\lambda_{31}+\lambda_{31}^{\prime}+2 \lambda_{1}+2 \lambda_{3}}
$$

with the following conditions:

- $\mu_{12}^{\prime 2}>\left(\lambda_{1}+\lambda_{3}-\lambda_{11}\right) v^{2}$

- $\mu_{12}^{\prime 2}>\left(\lambda_{1}+\lambda_{2}-\lambda_{22}\right) v^{2}$

- $\mu_{12}^{\prime 2}>\left(\lambda_{2}+\lambda_{3}-\lambda_{33}\right) v^{2}$

- $4\left(\mu_{12}^{\prime 2}-\left(\lambda_{1}+\lambda_{3}-\lambda_{11}\right) v^{2}\right)\left(\mu_{12}^{\prime 2}-\left(\lambda_{1}+\lambda_{2}-\lambda_{22}\right) v^{2}\right)>$

$$
\left(-2 \mu_{12}^{\prime 2}+\left(4 \lambda_{1}+\lambda_{12}+\lambda_{12}^{\prime}\right) v^{2}\right)^{2}
$$

- $\left(\mu_{12}^{\prime 2}-\left(\lambda_{1}+\lambda_{3}-\lambda_{11}\right) v^{2}\right)\left(\mu_{12}^{\prime 2}-\left(\lambda_{1}+\lambda_{2}-\lambda_{22}\right) v^{2}\right)\left(\mu_{12}^{\prime 2}-\left(\lambda_{2}+\lambda_{3}-\lambda_{33}\right) v^{2}\right)$

$$
\begin{aligned}
& +2\left(-\mu_{12}^{\prime 2}+\left(\frac{4 \lambda_{1}+\lambda_{12}+\lambda_{12}^{\prime}}{2}\right) v^{2}\right)\left(\frac{4 \lambda_{3}+\lambda_{31}+\lambda_{31}^{\prime}}{2}\right)\left(\frac{4 \lambda_{2}+\lambda_{23}+\lambda_{23}^{\prime}}{2}\right) v^{4}> \\
& \left(\mu_{12}^{\prime 2}-\left(\lambda_{1}+\lambda_{3}-\lambda_{11}\right) v^{2}\right)\left(-\mu_{12}^{\prime 2}+\left(\frac{4 \lambda_{1}+\lambda_{12}+\lambda_{12}^{\prime}}{2}\right) v^{2}\right)^{2} \\
& +\left(\mu_{12}^{\prime 2}-\left(\lambda_{1}+\lambda_{2}-\lambda_{22}\right) v^{2}\right)\left(\frac{4 \lambda_{3}+\lambda_{31}+\lambda_{31}^{\prime}}{2}\right)^{2} v^{4} \\
& +\left(\mu_{12}^{\prime 2}-\left(\lambda_{2}+\lambda_{3}-\lambda_{33}\right) v^{2}\right)\left(\frac{4 \lambda_{2}+\lambda_{23}+\lambda_{23}^{\prime}}{2}\right)^{2} v^{4}
\end{aligned}
$$

Higgs mass spectrum for $\left(0,0, \frac{v}{\sqrt{2}}\right)$

Expanding the potential around the vacuum point $\left(0,0, \frac{v}{\sqrt{2}}\right)$, with

$$
v^{2}=\frac{\mu_{3}^{2}}{\lambda_{33}}
$$

the mass spectrum appears as follows:

$$
\begin{aligned}
& \mathbf{A}_{3}: m^{2}=0 \\
& \mathbf{H}_{3}^{ \pm}: m^{2}=0 \\
& \mathbf{H}_{3}: m^{2}=2 \mu_{3}^{2} \\
& \mathbf{H}_{2} \equiv \frac{a H_{2}^{0}+H_{1}^{0}}{\sqrt{1+a^{2}}}: m^{2}=\frac{1}{2}(X-\sqrt{Y}) \\
& \mathbf{H}_{1} \equiv \frac{b H_{2}^{0}+H_{1}^{0}}{\sqrt{1+b^{2}}}: \quad m^{2}=\frac{1}{2}(X+\sqrt{Y}) \\
& \quad \text { where } \quad X=-\mu_{1}^{2}-\mu_{2}^{2}+\frac{1}{2}\left(\lambda_{23}+\lambda_{31}+\lambda_{23}^{\prime}+\lambda_{31}^{\prime}+2 \lambda_{2}+2 \lambda_{3}\right) v^{2} \\
& Y=4 \mu_{12}^{4}+\left[\mu_{1}^{2}-\mu_{2}^{2}+\frac{1}{2}\left(\lambda_{23}-\lambda_{31}+\lambda_{23}^{\prime}-\lambda_{31}^{\prime}+2 \lambda_{2}-2 \lambda_{3}\right) v^{2}\right]^{2} \\
& a=\frac{1}{-2 \mu_{12}^{2}}\left[\mu_{1}^{2}-\mu_{2}^{2}+\frac{1}{2}\left(\lambda_{23}-\lambda_{31}+\lambda_{23}^{\prime}-\lambda_{31}^{\prime}+2 \lambda_{2}-2 \lambda_{3}\right) v^{2}-\sqrt{Y}\right] \\
& b=\frac{1}{-2 \mu_{12}^{2}}\left[\mu_{1}^{2}-\mu_{2}^{2}+\frac{1}{2}\left(\lambda_{23}-\lambda_{31}+\lambda_{23}^{\prime}-\lambda_{31}^{\prime}+2 \lambda_{2}-2 \lambda_{3}\right) v^{2}+\sqrt{Y}\right]
\end{aligned}
$$




$$
\begin{aligned}
& \mathbf{H}_{2}^{ \pm}=\frac{a^{\prime} H_{2}^{ \pm}+H_{1}^{ \pm}}{\sqrt{1+a^{\prime 2}}}: \quad m^{2}=\frac{1}{2}\left(X^{\prime}-\sqrt{Y^{\prime}}\right) \\
& \mathbf{H}_{1}^{ \pm}=\frac{b^{\prime} H_{2}^{ \pm}+H_{1}^{ \pm}}{\sqrt{1+b^{\prime 2}}}: \quad m^{2}=\frac{1}{2}\left(X^{\prime}+\sqrt{Y^{\prime}}\right) \\
& \text { where } \quad X^{\prime}=-\mu_{2}^{2}-\mu_{1}^{2}+\frac{1}{2}\left(\lambda_{31}+\lambda_{23}\right) v^{2} \\
& Y^{\prime}=4 \mu_{12}^{4}+\left[\mu_{2}^{2}-\mu_{1}^{2}+\frac{1}{2}\left(\lambda_{31}-\lambda_{23}\right) v^{2}\right]^{2} \\
& a^{\prime}=\frac{1}{-2 \mu_{12}^{2}}\left[\mu_{2}^{2}-\mu_{1}^{2}+\frac{1}{2}\left(\lambda_{31}-\lambda_{23}\right) v^{2}-\sqrt{Y^{\prime}}\right] \\
& b^{\prime}=\frac{1}{-2 \mu_{12}^{2}}\left[\mu_{2}^{2}-\mu_{1}^{2}+\frac{1}{2}\left(\lambda_{31}-\lambda_{23}\right) v^{2}+\sqrt{Y^{\prime}}\right] \\
& \mathbf{A}_{2} \equiv \frac{a^{\prime \prime} A_{2}^{0}+A_{1}^{0}}{\sqrt{1+a^{\prime \prime 2}}}: \quad m^{2}=\frac{1}{2}\left(X^{\prime \prime}-\sqrt{Y^{\prime \prime}}\right) \\
& \mathbf{A}_{1} \equiv \frac{b^{\prime \prime} A_{2}^{0}+A_{1}^{0}}{\sqrt{1+b^{\prime \prime 2}}}: \quad m^{2}=\frac{1}{2}\left(X^{\prime \prime}+\sqrt{Y^{\prime \prime}}\right) \\
& \text { where } \quad X^{\prime \prime}=-\mu_{1}^{2}-\mu_{2}^{2}+\frac{1}{2}\left(\lambda_{23}+\lambda_{31}+\lambda_{23}^{\prime}+\lambda_{31}^{\prime}-2 \lambda_{2}-2 \lambda_{3}\right) v^{2} \\
& Y^{\prime \prime}=4 \mu_{12}^{4}+\left[\mu_{1}^{2}-\mu_{2}^{2}+\frac{1}{2}\left(\lambda_{23}-\lambda_{31}+\lambda_{23}^{\prime}-\lambda_{31}^{\prime}-2 \lambda_{2}+2 \lambda_{3}\right) v^{2}\right]^{2} \\
& a^{\prime \prime}=\frac{1}{-2 \mu_{12}^{2}}\left[\mu_{1}^{2}-\mu_{2}^{2}+\frac{1}{2}\left(\lambda_{23}-\lambda_{31}+\lambda_{23}^{\prime}-\lambda_{31}^{\prime}-2 \lambda_{2}+2 \lambda_{3}\right) v^{2}-\sqrt{Y^{\prime \prime}}\right] \\
& b^{\prime \prime}=\frac{1}{-2 \mu_{12}^{2}}\left[\mu_{1}^{2}-\mu_{2}^{2}+\frac{1}{2}\left(\lambda_{23}-\lambda_{31}+\lambda_{23}^{\prime}-\lambda_{31}^{\prime}-2 \lambda_{2}+2 \lambda_{3}\right) v^{2}+\sqrt{Y^{\prime \prime}}\right]
\end{aligned}
$$

The lightest neutral field from the first or the second doublet, stabilised by the conserved $Z_{2}$ symmetry, is a viable DM candidate.

\section{$3.5 \quad \mathrm{Z}_{3}$ symmetric $3 \mathrm{HDM}$ potential}

The $Z_{3}$-symmetric $3 \mathrm{HDM}$ potential contains the following terms:

$$
V_{Z_{3}}=\lambda_{1}\left(\phi_{1}^{\dagger} \phi_{2}\right)\left(\phi_{3}^{\dagger} \phi_{2}\right)+\lambda_{2}\left(\phi_{2}^{\dagger} \phi_{3}\right)\left(\phi_{1}^{\dagger} \phi_{3}\right)+\lambda_{3}\left(\phi_{3}^{\dagger} \phi_{1}\right)\left(\phi_{2}^{\dagger} \phi_{1}\right)+h . c .
$$

in addition to $V_{0}$ in eq. (3.2).

This group is generated by $a=\operatorname{diag}\left(\omega, \omega^{2}, 1\right)$, where $\omega=\exp (2 i \pi / 3)$.

The possible minima of this potential are:

1. Point $\left(0,0, \frac{v}{\sqrt{2}}\right)$ respects the symmetry of the potential and becomes the minimum at

$$
v^{2}=\frac{\mu_{3}^{2}}{\lambda_{33}}
$$

provided the following conditions are satisfied:

$$
\begin{array}{ll}
\text { - } & -\mu_{2}^{2}+\frac{1}{2}\left(\lambda_{23}+\lambda_{23}^{\prime}\right) v^{2}>0 \\
\text { - } & -\mu_{1}^{2}+\frac{1}{2}\left(\lambda_{31}+\lambda_{31}^{\prime}\right) v^{2}>0 \\
\text { - } & \mu_{3}^{2}>0
\end{array}
$$

The last condition is already required for the positivity of mass eigenstates at point $\left(0,0, \frac{v}{\sqrt{2}}\right)$. 
2. Point $\left(0, \frac{v}{\sqrt{2}}, \frac{v}{\sqrt{2}}\right)$ breaks the symmetry of the potential completely. This point becomes the minimum only when $\lambda_{1}=-\lambda_{2}$ (in the more general case of $v_{2} \neq v_{3}$ the condition $v_{2} / v_{3}=-\lambda_{2} / \lambda_{1}$ is required) which does not lead to an extra symmetry of the potential. The minimisation requires

$$
v^{2}=\frac{2 \mu_{3}^{2}}{2 \lambda_{33}+\lambda_{23}+\lambda_{23}^{\prime}}=\frac{2 \mu_{2}^{2}}{2 \lambda_{22}+\lambda_{23}+\lambda_{23}^{\prime}}
$$

and the following conditions be satisfied:

- $\lambda_{22}>0$

- $\lambda_{33}>0$

- $\quad-2 \mu_{1}^{2}+\left(\lambda_{12}+\lambda_{12}^{\prime}+\lambda_{31}+\lambda_{31}^{\prime}\right) v^{2}>0$

- $\lambda_{22}\left(-4 \mu_{1}^{2}+2\left(\lambda_{12}+\lambda_{12}^{\prime}+\lambda_{31}+\lambda_{31}^{\prime}\right) v^{2}\right)>\lambda_{1}^{2} v^{2}$

- $\lambda_{22} \lambda_{33}\left(-2 \mu_{1}^{2}+\left(\lambda_{12}+\lambda_{12}^{\prime}+\lambda_{31}+\lambda_{31}^{\prime}\right) v^{2}\right)+\lambda_{1} \lambda_{2}\left(\lambda_{23}+\lambda_{23}^{\prime}\right) v^{2}>$ $\frac{1}{2} \lambda_{33} \lambda_{1}^{2} v^{2}+2 \lambda_{22} \lambda_{2}^{2} v^{2}+\frac{1}{4}\left(\lambda_{23}+\lambda_{23}^{\prime}\right)^{2}\left(-2 \mu_{1}^{2}+2\left(\lambda_{12}+\lambda_{12}^{\prime}+\lambda_{31}+\lambda_{31}^{\prime}\right) v^{2}\right)$

3. Point $\left(\frac{v}{\sqrt{2}}, \frac{v}{\sqrt{2}}, \frac{v}{\sqrt{2}}\right)$ breaks the symmetry of the potential completely and becomes the minimum at

$$
\begin{aligned}
v^{2} & =\frac{2 \mu_{3}^{2}}{2 \lambda_{33}+\lambda_{23}+\lambda_{23}^{\prime}+\lambda_{31}+\lambda_{31}^{\prime}+\lambda_{1}+2 \lambda_{2}+\lambda_{3}} \\
& =\frac{2 \mu_{2}^{2}}{2 \lambda_{22}+\lambda_{12}+\lambda_{12}^{\prime}+\lambda_{23}+\lambda_{23}^{\prime}+2 \lambda_{1}+\lambda_{2}+\lambda_{3}} \\
& =\frac{2 \mu_{1}^{2}}{2 \lambda_{11}+\lambda_{12}+\lambda_{12}^{\prime}+\lambda_{31}+\lambda_{31}^{\prime}+\lambda_{1}+\lambda_{2}+2 \lambda_{3}}
\end{aligned}
$$

with the following conditions:

- $2 \lambda_{11}>\lambda_{1}+\lambda_{2}+2 \lambda_{3}$

- $2 \lambda_{22}>2 \lambda_{1}+\lambda_{2}+\lambda_{3}$

- $2 \lambda_{33}>\lambda_{1}+2 \lambda_{2}+\lambda_{3}$

- $\left(\lambda_{1}+\lambda_{2}+2 \lambda_{3}-2 \lambda_{11}\right)\left(2 \lambda_{1}+\lambda_{2}+\lambda_{3}-2 \lambda_{22}\right)>\left(\lambda_{1}+2 \lambda_{3}+\lambda_{12}+\lambda_{12}^{\prime}\right)^{2}$

- $\left(\lambda_{1}+\lambda_{2}+2 \lambda_{3}-2 \lambda_{11}\right)\left(2 \lambda_{1}+\lambda_{2}+\lambda_{3}-2 \lambda_{22}\right)\left(\lambda_{1}+2 \lambda_{2}+\lambda_{3}-2 \lambda_{33}\right)$

$$
\begin{aligned}
& +2\left(\lambda_{1}+2 \lambda_{3}+\lambda_{12}+\lambda_{12}^{\prime}\right)\left(2 \lambda_{2}+\lambda_{3}+\lambda_{31}+\lambda_{31}^{\prime}\right)\left(2 \lambda_{1}+\lambda_{2}+\lambda_{23}+\lambda_{23}^{\prime}\right)> \\
& -\left(\lambda_{1}+2 \lambda_{2}+\lambda_{3}-2 \lambda_{33}\right)\left(\lambda_{1}+2 \lambda_{3}+\lambda_{12}+\lambda_{12}^{\prime}\right)^{2} \\
& -\left(\lambda_{1}+\lambda_{2}+2 \lambda_{3}-2 \lambda_{11}\right)\left(2 \lambda_{1}+\lambda_{2}+\lambda_{23}+\lambda_{23}^{\prime}\right)^{2} \\
& -\left(2 \lambda_{1}+\lambda_{2}+\lambda_{3}-2 \lambda_{22}\right)\left(2 \lambda_{2}+\lambda_{3}+\lambda_{31}+\lambda_{31}^{\prime}\right)
\end{aligned}
$$


Higgs mass spectrum for $\left(0,0, \frac{v}{\sqrt{2}}\right)$

Expanding the potential around the vacuum point $\left(0,0, \frac{v}{\sqrt{2}}\right)$, with

$$
v^{2}=\frac{\mu_{3}^{2}}{\lambda_{33}}
$$

the mass spectrum is the same as the $\mathrm{U}(1)$-symmetric case with the slight difference in the definition of $a, b$ and $Y$ (replace $\lambda_{1}$ by $\lambda_{2}$ ).

The lightest neutral field from the first or the second doublet, stabilised by the conserved $Z_{3}$ symmetry, is a viable DM candidate.

\section{6 $\quad \mathrm{Z}_{4}$ symmetric $3 \mathrm{HDM}$ potential}

The most general $Z_{4}$-symmetric potential has two parts, $V_{0}$ in eq. (3.2) and the following terms:

$$
V_{Z_{4}}=\lambda_{1}\left(\phi_{3}^{\dagger} \phi_{1}\right)\left(\phi_{3}^{\dagger} \phi_{2}\right)+\lambda_{2}\left(\phi_{1}^{\dagger} \phi_{2}\right)^{2}+\text { h.c. }
$$

with generator $a=\operatorname{diag}(i,-i, 1)$.

The possible minima of this potential are:

1. Point $\left(0,0, \frac{v}{\sqrt{2}}\right)$ respects the symmetry of the potential and becomes the minimum at

$$
v^{2}=\frac{\mu_{3}^{2}}{\lambda_{33}}
$$

provided the following conditions are satisfied:

$$
\begin{array}{ll}
\text { - } & -\mu_{2}^{2}+\frac{1}{2}\left(\lambda_{23}+\lambda_{23}^{\prime}\right) v^{2}>0 \\
\text { - } & -\mu_{1}^{2}+\frac{1}{2}\left(\lambda_{31}+\lambda_{31}^{\prime}\right) v^{2}>0 \\
\text { - } & \mu_{3}^{2}>0
\end{array}
$$

The last condition is already required for the positivity of mass eigenstates at point $\left(0,0, \frac{v}{\sqrt{2}}\right)$.

2. Point $\left(0, \frac{v}{\sqrt{2}}, \frac{v}{\sqrt{2}}\right)$ only becomes the minimum when $\lambda_{1}=0$ which leads to the potential being symmetric under $U_{1}(1)$ and $Z_{2}$ generated by $(-1,-1,1)$. We therefore conclude that this VEV alignment is not realised in the $Z_{4}$-symmetric potential.

3. Point $\left(\frac{v}{\sqrt{2}}, \frac{v}{\sqrt{2}}, \frac{v}{\sqrt{2}}\right)$ breaks the symmetry of the potential completely and becomes the minimum at

$$
\begin{aligned}
v^{2} & =\frac{2 \mu_{3}^{2}}{2 \lambda_{33}+\lambda_{23}+\lambda_{23}^{\prime}+\lambda_{31}+\lambda_{31}^{\prime}+2 \lambda_{1}} \\
& =\frac{2 \mu_{2}^{2}}{2 \lambda_{22}+\lambda_{12}+\lambda_{12}^{\prime}+\lambda_{23}+\lambda_{23}^{\prime}+\lambda_{1}+2 \lambda_{2}}
\end{aligned}
$$




$$
=\frac{2 \mu_{1}^{2}}{2 \lambda_{11}+\lambda_{12}+\lambda_{12}^{\prime}+\lambda_{31}+\lambda_{31}^{\prime}+\lambda_{1}+2 \lambda_{2}}
$$

with the following conditions:

- $2 \lambda_{11}>\lambda_{1}+2 \lambda_{2}$

- $2 \lambda_{22}>\lambda_{1}+2 \lambda_{2}$

- $\lambda_{33}>\lambda_{1}$

- $\left(\lambda_{1}+2 \lambda_{2}-2 \lambda_{11}\right)\left(\lambda_{1}+2 \lambda_{2}-2 \lambda_{22}\right)>\left(4 \lambda_{2}+\lambda_{12}+\lambda_{12}^{\prime}\right)^{2}$

Higgs mass spectrum for $\left(0,0, \frac{v}{\sqrt{2}}\right)$

Expanding the potential around the vacuum point $\left(0,0, \frac{v}{\sqrt{2}}\right)$, with

$$
v^{2}=\frac{\mu_{3}^{2}}{\lambda_{33}}
$$

the mass spectrum is the same as the $\mathrm{U}(1)$-symmetric case.

The lightest neutral field from the first or the second doublet, stabilised by the conserved $Z_{4}$ symmetry, is a viable DM candidate.

\section{$3.7 \quad \mathrm{Z}_{2} \times \mathrm{Z}_{2}$ symmetric $3 \mathrm{HDM}$ potential}

The most general $Z_{2} \times Z_{2}$-symmetric potential consists of $V_{0}$ in eq. (3.2) and

$$
V_{Z_{2} \times Z_{2}}=\lambda_{1}\left(\phi_{1}^{\dagger} \phi_{2}\right)^{2}+\lambda_{2}\left(\phi_{2}^{\dagger} \phi_{3}\right)^{2}+\lambda_{3}\left(\phi_{3}^{\dagger} \phi_{1}\right)^{2}+h . c .
$$

generated by independent sign flips of the three doublets: $a_{1}=\operatorname{diag}(-1,1,1)$ and $a_{2}=$ $\operatorname{diag}(1,-1,1)$.

The possible minima of this potential are:

1. Point $\left(0,0, \frac{v}{\sqrt{2}}\right)$ respects the symmetry of the potential and becomes the minimum at

$$
v^{2}=\frac{\mu_{3}^{2}}{\lambda_{33}}
$$

provided the following conditions are satisfied:

$$
\begin{array}{ll}
\text { - } & -\mu_{2}^{2}+\frac{1}{2}\left(\lambda_{23}+\lambda_{23}^{\prime}\right) v^{2}>0 \\
\text { - } & -\mu_{1}^{2}+\frac{1}{2}\left(\lambda_{31}+\lambda_{31}^{\prime}\right) v^{2}>0 \\
\text { - } & \mu_{3}^{2}>0
\end{array}
$$

The last condition is already required for the positivity of mass eigenstates at point $\left(0,0, \frac{v}{\sqrt{2}}\right)$.

2. Point $\left(0, \frac{v}{\sqrt{2}}, \frac{v}{\sqrt{2}}\right)$ breaks the symmetry of the potential to $Z_{2}$ generated by $(-1,1,1)$ and becomes the minimum at

$$
v^{2}=\frac{2 \mu_{3}^{2}}{2 \lambda_{33}+\lambda_{23}+\lambda_{23}^{\prime}+\lambda_{31}+\lambda_{31}^{\prime}+2 \lambda_{2}+2 \lambda_{3}}
$$




$$
=\frac{2 \mu_{2}^{2}}{2 \lambda_{22}+\lambda_{12}+\lambda_{12}^{\prime}+\lambda_{23}+\lambda_{23}^{\prime}+2 \lambda_{1}+2 \lambda_{2}}
$$

when the following conditions are satisfied:

- $\quad-2 \mu_{1}^{2}+\left(\lambda_{12}+\lambda_{12}^{\prime}+\lambda_{31}+\lambda_{31}^{\prime}\right) v^{2}>0$

- $2 \lambda_{22}-2 \lambda_{1}-2 \lambda_{2}-\lambda_{12}-\lambda_{12}^{\prime}>0$

- $2 \lambda_{33}-2 \lambda_{2}-2 \lambda_{3}-\lambda_{31}-\lambda_{31}^{\prime}>0$

- $\left(2 \lambda_{22}-2 \lambda_{1}-2 \lambda_{2}-\lambda_{12}-\lambda_{12}^{\prime}\right)\left(2 \lambda_{33}-2 \lambda_{2}-2 \lambda_{3}-\lambda_{31}-\lambda_{31}^{\prime}\right)>$

$$
\left(\lambda_{23}+\lambda_{23}^{\prime}+4 \lambda_{2}\right)^{2} v^{4}
$$

This VEV alignment for the softly broken $Z_{2} \times Z_{2}$-symmetric $3 \mathrm{HDM}$ has been studied in detail in [26].

3. Point $\left(\frac{v}{\sqrt{2}}, \frac{v}{\sqrt{2}}, \frac{v}{\sqrt{2}}\right)$ breaks the symmetry of the potential completely and becomes the minimum at

$$
\begin{aligned}
v^{2} & =\frac{2 \mu_{3}^{2}}{2 \lambda_{33}+\lambda_{23}+\lambda_{23}^{\prime}+\lambda_{31}+\lambda_{31}^{\prime}+2 \lambda_{2}+2 \lambda_{3}} \\
& =\frac{2 \mu_{2}^{2}}{2 \lambda_{22}+\lambda_{12}+\lambda_{12}^{\prime}+\lambda_{23}+\lambda_{23}^{\prime}+2 \lambda_{1}+2 \lambda_{2}} \\
& =\frac{2 \mu_{1}^{2}}{2 \lambda_{11}+\lambda_{12}+\lambda_{12}^{\prime}+\lambda_{31}+\lambda_{31}^{\prime}+2 \lambda_{1}+2 \lambda_{3}}
\end{aligned}
$$

with the following conditions:

- $\lambda_{11}>\lambda_{1}+\lambda_{3}$

- $\lambda_{22}>\lambda_{1}+\lambda_{2}$

- $\lambda_{33}>\lambda_{2}+\lambda_{3}$

- $4\left(\lambda_{1}+\lambda_{3}-\lambda_{11}\right)\left(\lambda_{1}+\lambda_{2}-\lambda_{22}\right)>\left(2 \lambda_{1}+\lambda_{12}+\lambda_{12}^{\prime}\right)^{2}$

- $4\left(\lambda_{11}-\lambda_{1}-\lambda_{3}\right)\left(\lambda_{22}-\lambda_{1}-\lambda_{2}\right)\left(\lambda_{33}-\lambda_{2}-\lambda_{3}\right)$

$$
\begin{aligned}
& +\left(2 \lambda_{1}+\lambda_{12}+\lambda_{12}^{\prime}\right)\left(2 \lambda_{3}+\lambda_{31}+\lambda_{31}^{\prime}\right)\left(2 \lambda_{2}+\lambda_{23}+\lambda_{23}^{\prime}\right)> \\
& \left(\lambda_{33}-\lambda_{2}-\lambda_{3}\right)\left(2 \lambda_{1}+\lambda_{12}+\lambda_{12}^{\prime}\right)^{2}+\left(\lambda_{11}-\lambda_{1}-\lambda_{3}\right)\left(2 \lambda_{2}+\lambda_{23}+\lambda_{23}^{\prime}\right)^{2} \\
& +\left(\lambda_{22}-\lambda_{1}-\lambda_{2}\right)\left(2 \lambda_{3}+\lambda_{31}+\lambda_{31}^{\prime}\right)^{2}
\end{aligned}
$$

Higgs mass spectrum for $\left(0,0, \frac{v}{\sqrt{2}}\right)$

Expanding the potential around the vacuum point $\left(0,0, \frac{v}{\sqrt{2}}\right)$, with

$$
v^{2}=\frac{\mu_{3}^{2}}{\lambda_{33}}
$$

the mass spectrum appears as follows:

$$
\begin{array}{ll}
\mathbf{A}_{3}: & m^{2}=0 \\
\mathbf{H}_{3}^{ \pm}: & m^{2}=0
\end{array}
$$




\begin{tabular}{|l|l|l|}
\hline Symmetry & Generators & Potential \\
\hline$D_{6}$ & $\operatorname{diag}\left(\omega, \omega^{2}, 1\right), \quad \phi_{1} \leftrightarrow-\phi_{2}$ & $\begin{array}{l}V_{0}^{\prime}+\lambda_{1}\left[\left(\phi_{2}^{\dagger} \phi_{1}\right)\left(\phi_{3}^{\dagger} \phi_{1}\right)-\left(\phi_{1}^{\dagger} \phi_{2}\right)\left(\phi_{3}^{\dagger} \phi_{2}\right)\right]+ \\
\lambda_{2}\left(\phi_{1}^{\dagger} \phi_{3}\right)\left(\phi_{2}^{\dagger} \phi_{3}\right)+h . c .\end{array}$ \\
\hline$D_{8}$ & $\operatorname{diag}(i,-i, 1), \quad \phi_{1} \leftrightarrow-\phi_{2}$ & $V_{0}^{\prime}+\lambda_{1}\left(\phi_{3}^{\dagger} \phi_{1}\right)\left(\phi_{3}^{\dagger} \phi_{2}\right)+\lambda_{2}\left(\phi_{1}^{\dagger} \phi_{2}\right)^{2}+h . c$. \\
\hline$A_{4}$ & $\begin{array}{l}\operatorname{diag}(1,-1,-1), \\
\phi_{1} \rightarrow \phi_{2} \rightarrow \phi_{3} \rightarrow \phi_{1}\end{array}$ & $V_{0}^{\prime}+\lambda_{1}\left[\left(\phi_{1}^{\dagger} \phi_{2}\right)^{2}+\left(\phi_{2}^{\dagger} \phi_{3}\right)^{2}+\left(\phi_{3}^{\dagger} \phi_{1}\right)^{2}\right]+h . c$. \\
\hline$S_{4}$ & $\phi_{1} \leftrightarrow-\phi_{2}, \quad \phi_{1} \rightarrow \phi_{2} \rightarrow \phi_{3} \rightarrow \phi_{1}$ & $V_{0}^{\prime}+\lambda_{1}\left[\left(\phi_{1}^{\dagger} \phi_{2}\right)^{2}+\left(\phi_{2}^{\dagger} \phi_{3}\right)^{2}+\left(\phi_{3}^{\dagger} \phi_{1}\right)^{2}\right]+h . c$. \\
\hline$\Delta(54) / Z_{3}$ & $\begin{array}{l}\operatorname{diag}\left(\omega, \omega^{2}, 1\right), \quad \phi_{3} \rightarrow-\phi_{3}, \\
\phi_{1} \rightarrow \phi_{2} \rightarrow \phi_{3} \rightarrow \phi_{1}, \quad \phi_{1} \leftrightarrow \phi_{2}\end{array}$ & $\begin{array}{l}V_{0}^{\prime}+\lambda_{1}\left[\left(\phi_{1}^{\dagger} \phi_{2}\right)\left(\phi_{1}^{\dagger} \phi_{3}\right)+\left(\phi_{2}^{\dagger} \phi_{3}\right)\left(\phi_{2}^{\dagger} \phi_{1}\right)+\right. \\
\left.\left(\phi_{3}^{\dagger} \phi_{1}\right)\left(\phi_{3}^{\dagger} \phi_{2}\right)\right]+h . c .\end{array}$ \\
\hline$\Sigma(36)$ & $\operatorname{diag}\left(1, \omega, \omega^{2}\right), \quad d($ see eq. $(4.30))$ & $V_{0}^{\prime}$ \\
\hline
\end{tabular}

Table 2. All non-Abelian finite symmetries realisable in the scalar sector of 3HDMs. Note that in each case there are different constraints on the parameters of the phase invariant part of the potential, $V_{0}^{\prime}$, which are presented in the main text. The generators of the $\Sigma(36)$ symmetric potential are defined in detail in eq. (4.30).

$$
\begin{array}{ll}
\mathbf{H}_{3}: & m^{2}=2 \mu_{3}^{2} \\
\mathbf{H}_{2}^{ \pm}: & m^{2}=-\mu_{2}^{2}+\frac{1}{2} \lambda_{23} v^{2} \\
\mathbf{H}_{1}^{ \pm}: & m^{2}=-\mu_{1}^{2}+\frac{1}{2} \lambda_{31} v^{2} \\
\mathbf{H}_{2}: & m^{2}=-\mu_{2}^{2}+\frac{1}{2}\left(\lambda_{23}+\lambda_{23}^{\prime}+2 \lambda_{2}\right) v^{2} \\
\mathbf{A}_{2}: & m^{2}=-\mu_{2}^{2}+\frac{1}{2}\left(\lambda_{23}+\lambda_{23}^{\prime}-2 \lambda_{2}\right) v^{2} \\
\mathbf{H}_{1}: & m^{2}=-\mu_{1}^{2}+\frac{1}{2}\left(\lambda_{31}+\lambda_{31}^{\prime}+2 \lambda_{3}\right) v^{2} \\
\mathbf{A}_{1}: & m^{2}=-\mu_{1}^{2}+\frac{1}{2}\left(\lambda_{31}+\lambda_{31}^{\prime}-2 \lambda_{3}\right) v^{2}
\end{array}
$$

The lightest neutral field from the first or the second doublet which is stabilised by the conserved $Z_{2} \times Z_{2}$ symmetry is a viable DM candidate.

\section{Analysis of non-Abelian finite 3HDM}

In this section we study all non-Abelian finite symmetries in 3HDM potentials. Table 2 lists all these symmetry groups, their generators and their corresponding potentials.

\section{1 $\mathrm{D}_{6} \simeq \mathrm{Z}_{3} \rtimes \mathrm{Z}_{2}$ symmetric $3 \mathrm{HDM}$ potential}

The generic $D_{6}$-symmetric potential has the following form:

$$
\begin{aligned}
V_{D_{6}}= & -\mu_{12}^{2}\left(\phi_{1}^{\dagger} \phi_{1}+\phi_{2}^{\dagger} \phi_{2}\right)-\mu_{3}^{2}\left(\phi_{3}^{\dagger} \phi_{3}\right) \\
& +\lambda_{11}\left[\left(\phi_{1}^{\dagger} \phi_{1}\right)^{2}+\left(\phi_{2}^{\dagger} \phi_{2}\right)^{2}\right]+\lambda_{33}\left[\left(\phi_{3}^{\dagger} \phi_{3}\right)^{2}\right]
\end{aligned}
$$




$$
\begin{aligned}
& +\lambda_{12}\left[\left(\phi_{1}^{\dagger} \phi_{1}\right)\left(\phi_{2}^{\dagger} \phi_{2}\right)\right]+\lambda_{13}\left[\left(\phi_{3}^{\dagger} \phi_{3}\right)\left(\phi_{1}^{\dagger} \phi_{1}\right)+\left(\phi_{3}^{\dagger} \phi_{3}\right)\left(\phi_{2}^{\dagger} \phi_{2}\right)\right] \\
& +\lambda_{12}^{\prime}\left[\left(\phi_{1}^{\dagger} \phi_{2}\right)\left(\phi_{2}^{\dagger} \phi_{1}\right)\right]+\lambda_{13}^{\prime}\left[\left(\phi_{2}^{\dagger} \phi_{3}\right)\left(\phi_{3}^{\dagger} \phi_{2}\right)+\left(\phi_{3}^{\dagger} \phi_{1}\right)\left(\phi_{1}^{\dagger} \phi_{3}\right)\right] \\
& +\lambda_{1}\left[\left(\phi_{2}^{\dagger} \phi_{1}\right)\left(\phi_{3}^{\dagger} \phi_{1}\right)-\left(\phi_{1}^{\dagger} \phi_{2}\right)\left(\phi_{3}^{\dagger} \phi_{2}\right)\right]+\lambda_{2}\left[\left(\phi_{1}^{\dagger} \phi_{3}\right)\left(\phi_{2}^{\dagger} \phi_{3}\right)\right]+\text { h.c. }
\end{aligned}
$$

with real $\lambda_{1}$ and complex $\lambda_{2}$. This group is generated by

$$
a=\left(\begin{array}{ccc}
\omega & 0 & 0 \\
0 & \omega^{2} & 0 \\
0 & 0 & 1
\end{array}\right), \quad \omega=\exp (2 i \pi / 3), \quad b=\left(\begin{array}{ccc}
0 & -1 & 0 \\
-1 & 0 & 0 \\
0 & 0 & 1
\end{array}\right)
$$

with $a^{3}=1$ and $b^{2}=1[27]$.

The possible minima of this potential are:

1. Point $\left(0,0, \frac{v}{\sqrt{2}}\right)$ respects the symmetry of the potential and becomes the minimum at

$$
v^{2}=\frac{\mu_{3}^{2}}{\lambda_{33}}
$$

provided the following conditions are satisfied:

$$
\begin{array}{ll}
\text { - } & -\mu_{12}^{2}+\frac{1}{2}\left(\lambda_{23}+\lambda_{23}^{\prime}\right) v^{2}>0 \\
\text { - } & \mu_{3}^{2}>0
\end{array}
$$

The last condition is already required for the positivity of mass eigenstates at point $\left(0,0, \frac{v}{\sqrt{2}}\right)$.

2. Point $\left(0, \frac{v}{\sqrt{2}}, \frac{v}{\sqrt{2}}\right)$ breaks the symmetry of the potential completely and becomes the minimum only when $\lambda_{1}=\lambda_{2}$ (in the more general case of $v_{2} \neq v_{3}$ the condition $v_{2} / v_{3}=\lambda_{2} / \lambda_{1}$ is required) which does not lead to an extra symmetry of the potential. The minimisation requires

$$
v^{2}=\frac{2 \mu_{3}^{2}}{2 \lambda_{33}+\lambda_{13}+\lambda_{13}^{\prime}}=\frac{2 \mu_{12}^{2}}{2 \lambda_{11}+\lambda_{13}+\lambda_{13}^{\prime}}
$$

and the following conditions be satisfied:

- $\lambda_{11}>0$

- $\lambda_{33}>0$

- $\quad-2 \mu_{12}^{2}+\left(\lambda_{12}+\lambda_{12}^{\prime}+\lambda_{13}+\lambda_{13}^{\prime}\right) v^{2}>0$

- $\lambda_{11} \lambda_{33}\left(-2 \mu_{12}^{2}+\left(\lambda_{12}+\lambda_{12}^{\prime}+\lambda_{13}+\lambda_{13}^{\prime}\right) v^{2}\right)-\lambda_{1} \lambda_{2}\left(\lambda_{13}+\lambda_{13}^{\prime}\right) v^{2}>$

$$
\frac{1}{2} \lambda_{33} \lambda_{1}^{2} v^{2}+2 \lambda_{11} \lambda_{2}^{2} v^{2}+\frac{1}{4}\left(\lambda_{13}+\lambda_{13}^{\prime}\right)^{2}\left(-2 \mu_{12}^{2}+2\left(\lambda_{12}+\lambda_{12}^{\prime}+\lambda_{13}+\lambda_{13}^{\prime}\right) v^{2}\right)
$$


3. Point $\left(\frac{v}{\sqrt{2}}, \frac{v}{\sqrt{2}}, \frac{v}{\sqrt{2}}\right)$ becomes the minimum at

$$
\begin{aligned}
v^{2} & =\frac{2 \mu_{3}^{2}}{2 \lambda_{33}+2 \lambda_{13}+2 \lambda_{13}^{\prime}+2 \lambda_{2}} \\
& =\frac{2 \mu_{12}^{2}}{2 \lambda_{11}+\lambda_{12}+\lambda_{12}^{\prime}+\lambda_{13}+\lambda_{13}^{\prime} \pm \lambda_{1}+\lambda_{2}}
\end{aligned}
$$

which means that $\lambda_{1}=0$, however this condition leads to an extra $Z_{2}$ (the exchange of $\phi_{1}$ and $\phi_{2}$ ) symmetry of the potential. Therefore, we conclude that the point $\left(\frac{v}{\sqrt{2}}, \frac{v}{\sqrt{2}}, \frac{v}{\sqrt{2}}\right)$ cannot be a minimum of this potential.

Higgs mass spectrum for $\left(0,0, \frac{v}{\sqrt{2}}\right)$

Expanding the potential around this vacuum point, with

$$
v^{2}=\frac{\mu_{3}^{2}}{\lambda_{33}}
$$

the mass spectrum appears as follows:

$$
\begin{array}{ll}
\mathbf{A}_{3}: & m^{2}=0 \\
\mathbf{H}_{3}^{ \pm}: & m^{2}=0 \\
\mathbf{H}_{3}: & m^{2}=2 \mu_{3}^{2} \\
\mathbf{H}_{2}^{ \pm}: & m^{2}=-\mu_{12}^{2}+\frac{1}{2} \lambda_{13} v^{2} \\
\mathbf{H}_{1}^{ \pm}: & m^{2}=-\mu_{12}^{2}+\frac{1}{2} \lambda_{13} v^{2} \\
\mathbf{H}_{2} \equiv \frac{H_{2}^{0}-H_{1}^{0}}{\sqrt{2}}: \quad m^{2}=-\mu_{12}^{2}+\frac{1}{2}\left(\lambda_{13}+\lambda_{13}^{\prime}-\lambda_{2}\right) v^{2} \\
\mathbf{H}_{1} \equiv \frac{H_{2}^{0}+H_{1}^{0}}{\sqrt{2}}: \quad m^{2}=-\mu_{12}^{2}+\frac{1}{2}\left(\lambda_{13}+\lambda_{13}^{\prime}+\lambda_{2}\right) v^{2} \\
\mathbf{A}_{2} \equiv \frac{A_{2}^{0}-A_{1}^{0}}{\sqrt{2}}: \quad m^{2}=-\mu_{12}^{2}+\frac{1}{2}\left(\lambda_{13}+\lambda_{13}^{\prime}-\lambda_{2}\right) v^{2} \\
\mathbf{A}_{1} \equiv \frac{A_{2}^{0}+A_{1}^{0}}{\sqrt{2}}: \quad m^{2}=-\mu_{12}^{2}+\frac{1}{2}\left(\lambda_{13}+\lambda_{13}^{\prime}+\lambda_{2}\right) v^{2}
\end{array}
$$

The lightest neutral field from the first or the second doublet, stabilised by the conserved $D_{6}$ symmetry, is a viable DM candidate.

\section{2 $\mathrm{D}_{8} \simeq \mathrm{Z}_{4} \rtimes \mathrm{Z}_{2}$ symmetric $3 \mathrm{HDM}$ potential}

The generic $D_{8}$-symmetric $3 \mathrm{HDM}$ potential has the following form:

$$
\begin{aligned}
V_{D_{8}}= & -\mu_{12}^{2}\left(\phi_{1}^{\dagger} \phi_{1}+\phi_{2}^{\dagger} \phi_{2}\right)-\mu_{3}^{2}\left(\phi_{3}^{\dagger} \phi_{3}\right) \\
& +\lambda_{11}\left[\left(\phi_{1}^{\dagger} \phi_{1}\right)^{2}+\left(\phi_{2}^{\dagger} \phi_{2}\right)^{2}\right]+\lambda_{33}\left[\left(\phi_{3}^{\dagger} \phi_{3}\right)^{2}\right] \\
& +\lambda_{12}\left[\left(\phi_{1}^{\dagger} \phi_{1}\right)\left(\phi_{2}^{\dagger} \phi_{2}\right)\right]+\lambda_{13}\left[\left(\phi_{3}^{\dagger} \phi_{3}\right)\left(\phi_{1}^{\dagger} \phi_{1}\right)+\left(\phi_{3}^{\dagger} \phi_{3}\right)\left(\phi_{2}^{\dagger} \phi_{2}\right)\right] \\
& +\lambda_{12}^{\prime}\left[\left(\phi_{1}^{\dagger} \phi_{2}\right)\left(\phi_{2}^{\dagger} \phi_{1}\right)\right]+\lambda_{13}^{\prime}\left[\left(\phi_{2}^{\dagger} \phi_{3}\right)\left(\phi_{3}^{\dagger} \phi_{2}\right)+\left(\phi_{3}^{\dagger} \phi_{1}\right)\left(\phi_{1}^{\dagger} \phi_{3}\right)\right] \\
& +\lambda_{1}\left(\phi_{3}^{\dagger} \phi_{1}\right)\left(\phi_{3}^{\dagger} \phi_{2}\right)+\lambda_{2}\left(\phi_{1}^{\dagger} \phi_{2}\right)^{2}+\text { h.c. }
\end{aligned}
$$


This group is generated by:

$$
a=\left(\begin{array}{ccc}
i & 0 & 0 \\
0 & -i & 0 \\
0 & 0 & 1
\end{array}\right), \quad b=\left(\begin{array}{ccc}
0 & -1 & 0 \\
-1 & 0 & 0 \\
0 & 0 & 1
\end{array}\right)
$$

with $a^{4}=1, b^{2}=1$.

The possible minima of this potential are:

1. Point $\left(0,0, \frac{v}{\sqrt{2}}\right)$ respects the symmetry of the potential and becomes the minimum at

$$
v^{2}=\frac{\mu_{3}^{2}}{\lambda_{33}}
$$

provided the following conditions are satisfied:

$$
\begin{array}{ll}
\text { - } & -\mu_{12}^{2}+\frac{1}{2}\left(\lambda_{13}+\lambda_{13}^{\prime}\right) v^{2}>0 \\
\text { - } & \mu_{3}^{2}>0
\end{array}
$$

The last condition is already required for the positivity of mass eigenstates at point $\left(0,0, \frac{v}{\sqrt{2}}\right)$.

2. Point $\left(0, \frac{v}{\sqrt{2}}, \frac{v}{\sqrt{2}}\right)$ only becomes the minimum when $\lambda_{1}=0$ which leads to an extra $Z_{2}$ (the exchange of $\phi_{1}$ and $\phi_{2}$ ) symmetry of the potential. We therefore conclude that this VEV alignment is not realised in the $D_{8}$-symmetric potential.

3. Point $\left(\frac{v}{\sqrt{2}}, \frac{v}{\sqrt{2}}, \frac{v}{\sqrt{2}}\right)$ breaks the symmetry of the potential completely and becomes the minimum at

$$
\begin{aligned}
v^{2} & =\frac{2 \mu_{3}^{2}}{2 \lambda_{33}+2 \lambda_{13}+2 \lambda_{13}^{\prime}+2 \lambda_{1}} \\
& =\frac{2 \mu_{12}^{2}}{2 \lambda_{11}+\lambda_{12}+\lambda_{12}^{\prime}+\lambda_{13}+\lambda_{13}^{\prime}+\lambda_{1}+2 \lambda_{2}}
\end{aligned}
$$

with the following conditions:

- $\lambda_{33}>\lambda_{1}$

- $\left(4 \lambda_{2}+\lambda_{12}+\lambda_{12}^{\prime}\right)^{2}<\left(\lambda_{1}+2 \lambda_{2}-2 \lambda_{11}\right)^{2}$

- $2 \lambda_{11}>\lambda_{1}+2 \lambda_{2}$

- $\left(\lambda_{1}-\lambda_{33}\right)\left[\left(\lambda_{1}+2 \lambda_{2}-2 \lambda_{11}\right)^{2}-\left(\lambda_{12}+\lambda_{12}^{\prime}+4 \lambda_{2}\right)^{2}\right]<$

$$
\left(2 \lambda_{1}+\lambda_{13}+\lambda_{13}^{\prime}\right)^{2}\left(\lambda_{12}+\lambda_{12}^{\prime}+6 \lambda_{2}-2 \lambda_{11}+\lambda_{1}\right)
$$

Higgs mass spectrum for $\left(0,0, \frac{v}{\sqrt{2}}\right)$

Expanding the potential around the vacuum point $\left(0,0, \frac{v}{\sqrt{2}}\right)$, with

$$
v^{2}=\frac{\mu_{3}^{2}}{\lambda_{33}}
$$


the mass spectrum is the same as the $D_{6}$-symmetric case with the slight difference in the definition of $a, b$ and $Y$ (replace $\lambda_{2}$ by $\lambda_{1}$ ).

The lightest neutral field from the first or the second doublet, stabilised by the conserved $D_{8}$ symmetry, is a viable DM candidate.

\section{3 $\quad \mathrm{A}_{4} \simeq \mathrm{T}=\left(\mathrm{Z}_{2} \times \mathrm{Z}_{2}\right) \rtimes \mathrm{Z}_{3}$ symmetric $3 \mathrm{HDM}$ potential}

The $A_{4}$-symmetric 3 HDM can be represented by the following potential

$$
\begin{aligned}
V_{A_{4}}= & -\mu^{2}\left[\left(\phi_{1}^{\dagger} \phi_{1}\right)+\left(\phi_{2}^{\dagger} \phi_{2}\right)+\left(\phi_{3}^{\dagger} \phi_{3}\right)\right]+\lambda_{11}\left[\left(\phi_{1}^{\dagger} \phi_{1}\right)+\left(\phi_{2}^{\dagger} \phi_{2}\right)+\left(\phi_{3}^{\dagger} \phi_{3}\right)\right]^{2} \\
& +\lambda_{12}\left[\left(\phi_{1}^{\dagger} \phi_{1}\right)\left(\phi_{2}^{\dagger} \phi_{2}\right)+\left(\phi_{2}^{\dagger} \phi_{2}\right)\left(\phi_{3}^{\dagger} \phi_{3}\right)+\left(\phi_{3}^{\dagger} \phi_{3}\right)\left(\phi_{1}^{\dagger} \phi_{1}\right)\right] \\
& +\lambda_{12}^{\prime}\left(\left|\phi_{1}^{\dagger} \phi_{2}\right|^{2}+\left|\phi_{2}^{\dagger} \phi_{3}\right|^{2}+\left|\phi_{3}^{\dagger} \phi_{1}\right|^{2}\right)+\lambda_{1}\left[\left(\phi_{1}^{\dagger} \phi_{2}\right)^{2}+\left(\phi_{2}^{\dagger} \phi_{3}\right)^{2}+\left(\phi_{3}^{\dagger} \phi_{1}\right)^{2}\right]+\text { h.c. }
\end{aligned}
$$

with complex $\lambda_{1}$.

This potential is symmetric under:

$$
a=\left(\begin{array}{ccc}
1 & 0 & 0 \\
0 & -1 & 0 \\
0 & 0 & -1
\end{array}\right), \quad b=\left(\begin{array}{lll}
0 & 1 & 0 \\
0 & 0 & 1 \\
1 & 0 & 0
\end{array}\right)
$$

with $a^{2}=1, b^{3}=1$.

The possible minima of this potential are:

1. Point $\left(0,0, \frac{v}{\sqrt{2}}\right)$ breaks the symmetry of the potential to $Z_{2}$ generated by $g=$ $(-1,-1,1)$ and becomes the minimum at

$$
v^{2}=\frac{\mu_{3}^{2}}{\lambda_{33}}
$$

provided the following conditions are satisfied:

$$
\begin{aligned}
& \text { - }-2 \mu^{2}+\left(\lambda_{12}+\lambda_{12}^{\prime}+2 \lambda_{11}\right) v^{2}>0 \\
& \text { - } \mu^{2}>0
\end{aligned}
$$

The last condition is already required for the positivity of mass eigenstates at point $\left(0,0, \frac{v}{\sqrt{2}}\right)$.

2. Point $\left(0, \frac{v}{\sqrt{2}}, \frac{v}{\sqrt{2}}\right)$ becomes the minimum only when $\lambda_{1}$ is real which leads to an $S_{4}$-symmetric potential . Therefore, this point cannot be a minimum of the $A_{4^{-}}$ symmetric potential. Note that in the more general case, the point $\left(0, v_{2}, v_{3}\right)$ can become a minimum if the following condition is satisfied:

$$
\left(\frac{v_{2}}{v_{3}}\right)^{2}=\frac{\lambda_{12}+\lambda_{12}^{\prime}+2 \lambda_{1}^{*}}{\lambda_{12}+\lambda_{12}^{\prime}+2 \lambda_{1}}
$$


3. Point $\left(\frac{v}{\sqrt{2}}, \frac{v}{\sqrt{2}}, \frac{v}{\sqrt{2}}\right)$ breaks the symmetry of the potential to $S_{3}$ (permutation of the three doublets) and becomes the minimum at

$$
v^{2}=\frac{\mu^{2}}{3 \lambda_{11}+\lambda_{12}+\lambda_{12}^{\prime}+\lambda_{1}+\lambda_{1}^{*}}
$$

with the following conditions:

- $\quad-\lambda_{11}>2 \operatorname{Re} \lambda_{1}$

- $4\left(2 \operatorname{Re} \lambda_{1}+\lambda_{11}\right)^{2}>\left(\lambda_{12}+\lambda_{12}^{\prime}+2 \lambda_{11}+4 \operatorname{Re} \lambda_{1}\right)^{2}+\left(4 \operatorname{Im} \lambda_{1}\right)^{2}$

- $-4\left(\lambda_{11}+2 \operatorname{Re} \lambda_{1}\right)^{3}+3\left(\lambda_{11}+2 \operatorname{Re} \lambda_{1}\right)\left(\left(\lambda_{12}+\lambda_{12}^{\prime}+2 \lambda_{11}+4 \operatorname{Re} \lambda_{1}\right)^{2}+\left(4 \operatorname{Im} \lambda_{1}\right)^{2}\right)>$

$$
-\left(\left(\lambda_{12}+\lambda_{12}^{\prime}+2 \lambda_{11}+4 \operatorname{Re} \lambda_{1}\right)^{2}+\left(4 \operatorname{Im} \lambda_{1}\right)^{2}\right)\left(\lambda_{12}+\lambda_{12}^{\prime}+2 \lambda_{11}+4 \operatorname{Re} \lambda_{1}\right)
$$

Higgs mass spectrum for $\left(0,0, \frac{v}{\sqrt{2}}\right)$

Expanding the potential around $\left(0,0, \frac{v}{\sqrt{2}}\right)$, with

$$
v^{2}=\frac{\mu^{2}}{\lambda_{11}}
$$

we get

$$
\begin{array}{ll}
\mathbf{A}_{3}: & m^{2}=0 \\
\mathbf{H}_{3}^{ \pm}: & m^{2}=0 \\
\mathbf{H}_{3}: & m^{2}=2 \mu^{2} \\
\mathbf{H}_{2}^{ \pm}: & m^{2}=-\mu^{2}+\frac{1}{2}\left(\lambda_{12}+2 \lambda_{11}\right) v^{2} \\
\mathbf{H}_{1}^{ \pm}: & m^{2}=-\mu^{2}+\frac{1}{2}\left(\lambda_{12}+2 \lambda_{11}\right) v^{2} \\
\mathbf{H}_{2} \equiv \frac{a H_{2}^{0}+A_{2}^{0}}{\sqrt{1+a^{2}}}: & m^{2}=-\mu^{2}+\frac{1}{2}\left(\lambda_{12}+\lambda_{12}^{\prime}+2 \lambda_{11}-\sqrt{\operatorname{Re}^{2} \lambda_{1}+\operatorname{Im}^{2} \lambda_{1}}\right) v^{2} \\
\mathbf{A}_{2} \equiv \frac{b H_{2}^{0}+A_{2}^{0}}{\sqrt{1+b^{2}}}: & m^{2}=-\mu^{2}+\frac{1}{2}\left(\lambda_{12}+\lambda_{12}^{\prime}+2 \lambda_{11}+\sqrt{\operatorname{Re}^{2} \lambda_{1}+\operatorname{Im}^{2} \lambda_{1}}\right) v^{2} \\
\mathbf{H}_{1} \equiv \frac{a H_{1}^{0}+A_{1}^{0}}{\sqrt{1+a^{2}}}: & m^{2}=-\mu^{2}+\frac{1}{2}\left(\lambda_{12}+\lambda_{12}^{\prime}+2 \lambda_{11}-\sqrt{\operatorname{Re}^{2} \lambda_{1}+\operatorname{Im}^{2} \lambda_{1}}\right) v^{2} \\
\mathbf{A}_{1} \equiv \frac{b H_{1}^{0}+A_{1}^{0}}{\sqrt{1+b^{2}}}: & m^{2}=-\mu^{2}+\frac{1}{2}\left(\lambda_{12}+\lambda_{12}^{\prime}+2 \lambda_{11}+\sqrt{\operatorname{Re}^{2} \lambda_{1}+\operatorname{Im}^{2} \lambda_{1}}\right) v^{2} \\
\text { where } & a=\frac{1}{\operatorname{Im} \lambda_{1}}\left[\operatorname{Re} \lambda_{1}-\sqrt{\operatorname{Re}^{2} \lambda_{1}+\operatorname{Im}^{2} \lambda_{1}}\right] \\
& b=\frac{1}{\operatorname{Im} \lambda_{1}}\left[\operatorname{Re} \lambda_{1}+\sqrt{\operatorname{Re}^{2} \lambda_{1}+\operatorname{Im}^{2} \lambda_{1}}\right]
\end{array}
$$

The lightest neutral field from the first or the second doublet, stabilised by the remaining $Z_{2}$ symmetry, is a viable DM candidate.

\section{4 $\mathrm{S}_{4} \simeq \mathrm{O}=\left(\mathrm{Z}_{2} \times \mathrm{Z}_{2}\right) \rtimes \mathrm{S}_{3}$ symmetric $3 \mathrm{HDM}$ potential}

The $S_{4}$-symmetric $3 \mathrm{HDM}$ can be represented by the following potential

$$
V_{S_{4}}=-\mu^{2}\left[\left(\phi_{1}^{\dagger} \phi_{1}\right)+\left(\phi_{2}^{\dagger} \phi_{2}\right)+\left(\phi_{3}^{\dagger} \phi_{3}\right)\right]+\lambda_{11}\left[\left(\phi_{1}^{\dagger} \phi_{1}\right)+\left(\phi_{2}^{\dagger} \phi_{2}\right)+\left(\phi_{3}^{\dagger} \phi_{3}\right)\right]^{2}
$$




$$
\begin{aligned}
& +\lambda_{12}\left[\left(\phi_{1}^{\dagger} \phi_{1}\right)\left(\phi_{2}^{\dagger} \phi_{2}\right)+\left(\phi_{2}^{\dagger} \phi_{2}\right)\left(\phi_{3}^{\dagger} \phi_{3}\right)+\left(\phi_{3}^{\dagger} \phi_{3}\right)\left(\phi_{1}^{\dagger} \phi_{1}\right)\right] \\
& +\lambda_{12}^{\prime}\left(\left|\phi_{1}^{\dagger} \phi_{2}\right|^{2}+\left|\phi_{2}^{\dagger} \phi_{3}\right|^{2}+\left|\phi_{3}^{\dagger} \phi_{1}\right|^{2}\right)+\lambda_{1}\left[\left(\phi_{1}^{\dagger} \phi_{2}\right)^{2}+\left(\phi_{2}^{\dagger} \phi_{3}\right)^{2}+\left(\phi_{3}^{\dagger} \phi_{1}\right)^{2}\right]+\text { h.c. }
\end{aligned}
$$

with real $\lambda_{1}$.

This potential is symmetric under:

$$
b=\left(\begin{array}{lll}
0 & 1 & 0 \\
0 & 0 & 1 \\
1 & 0 & 0
\end{array}\right), \quad c=\left(\begin{array}{ccc}
0 & -1 & 0 \\
-1 & 0 & 0 \\
0 & 0 & 1
\end{array}\right)
$$

with $b^{3}=1$ and $c^{2}=1$.

The possible minima of this potential are:

1. Point $\left(0,0, \frac{v}{\sqrt{2}}\right)$ breaks the symmetry of the potential to $Z_{2}$ generated by $c=\left(\begin{array}{ccc}0 & -1 & 0 \\ -1 & 0 & 0 \\ 0 & 0 & 1\end{array}\right)$ and becomes the minimum at

$$
v^{2}=\frac{\mu_{3}^{2}}{\lambda_{33}}
$$

provided the following conditions are satisfied:

$$
\begin{aligned}
& \text { - }-2 \mu^{2}+\left(\lambda_{12}+\lambda_{12}^{\prime}+2 \lambda_{11}\right) v^{2}>0 \\
& \text { - } \mu^{2}>0
\end{aligned}
$$

The last condition is already required for the positivity of mass eigenstates at point $\left(0,0, \frac{v}{\sqrt{2}}\right)$.

2. Point $\left(0, \frac{v}{\sqrt{2}}, \frac{v}{\sqrt{2}}\right)$ breaks the symmetry of the potential completely and becomes the minimum at

$$
v^{2}=\frac{2 \mu^{2}}{3 \lambda_{11}+\lambda_{12}+\lambda_{12}^{\prime}+2 \lambda_{1}}
$$

when the following conditions are satisfied:

$$
\begin{array}{ll}
\text { - } & 2 \lambda_{1}+\lambda_{11}<0 \\
\text { - } & \lambda_{12}+\lambda_{12}^{\prime}+4 \lambda_{1}<0 \\
\text { - } & 4 \lambda_{11}\left(\lambda_{11}+\lambda_{12}+\lambda_{12}^{\prime}+4 \lambda_{1}\right)<0
\end{array}
$$

3. Point $\left(\frac{v}{\sqrt{2}}, \frac{v}{\sqrt{2}}, \frac{v}{\sqrt{2}}\right)$ breaks the symmetry of the potential to $S_{3}$ (permutation of the three doublets) and becomes the minimum at

$$
v^{2}=\frac{\mu^{2}}{\lambda_{12}+\lambda_{12}^{\prime}+3 \lambda_{11}+2 \lambda_{1}}
$$

with the following conditions:

- $\lambda_{11}>2 \lambda_{1}$

- $\left(2 \lambda_{1}+\lambda_{12}+\lambda_{12}^{\prime}+2 \lambda_{11}\right)^{2}<4\left(2 \lambda_{1}-\lambda_{11}\right)^{2}$

- $\quad-4\left(\lambda_{11}+2 \lambda_{1}\right)^{3}+\left(2 \lambda_{1}+\lambda_{12}+2 \lambda_{11}+\lambda_{12}^{\prime}\right)^{3}>$

$$
3\left(-\lambda_{11}-2 \lambda_{1}\right)\left(2 \lambda_{1}+\lambda_{12}+2 \lambda_{11}+\lambda_{12}^{\prime}\right)^{2}
$$


Higgs mass spectrum for $\left(0,0, \frac{v}{\sqrt{2}}\right)$

Expanding the potential around $\left(0,0, \frac{v}{\sqrt{2}}\right)$, with

$$
v^{2}=\frac{\mu^{2}}{\lambda_{11}}
$$

the mass spectrum gets the following form:

$$
\begin{array}{ll}
\mathbf{A}_{3}: & m^{2}=0 \\
\mathbf{H}_{3}^{ \pm}: & m^{2}=0 \\
\mathbf{H}_{3}: & m^{2}=2 \mu^{2} \\
\mathbf{H}_{2}^{ \pm}: & m^{2}=-\mu^{2}+\frac{1}{2}\left(\lambda_{12}+2 \lambda_{11}\right) v^{2} \\
\mathbf{H}_{1}^{ \pm}: & m^{2}=-\mu^{2}+\frac{1}{2}\left(\lambda_{12}+2 \lambda_{11}\right) v^{2} \\
\mathbf{H}_{2}: & m^{2}=-\mu^{2}+\frac{1}{2}\left(\lambda_{12}+\lambda_{12}^{\prime}+2 \lambda_{11}+2 \lambda_{1}\right) v^{2} \\
\mathbf{A}_{2}: & m^{2}=-\mu^{2}+\frac{1}{2}\left(\lambda_{12}+\lambda_{12}^{\prime}+2 \lambda_{11}-2 \lambda_{1}\right) v^{2} \\
\mathbf{H}_{1}: & m^{2}=-\mu^{2}+\frac{1}{2}\left(\lambda_{12}+\lambda_{12}^{\prime}+2 \lambda_{11}+2 \lambda_{1}\right) v^{2} \\
\mathbf{A}_{1}: & m^{2}=-\mu^{2}+\frac{1}{2}\left(\lambda_{12}+\lambda_{12}^{\prime}+2 \lambda_{11}-2 \lambda_{1}\right) v^{2}
\end{array}
$$

The lightest neutral field from the first or the second doublet, stabilised by the remaining $Z_{2}$ symmetry, is a viable DM candidate.

\section{$4.5 \Delta(54) / \mathrm{Z}_{3} \simeq\left(\mathrm{Z}_{3} \times \mathrm{Z}_{3}\right) \rtimes \mathrm{Z}_{2}$ symmetric $3 \mathrm{HDM}$ potential}

A $3 \mathrm{HDM}$ potential symmetric under this group has the following form: ${ }^{9}$

$$
\begin{aligned}
V_{\Delta(54) / Z_{3}=} & -\mu^{2}\left[\phi_{1}^{\dagger} \phi_{1}+\phi_{2}^{\dagger} \phi_{2}+\phi_{3}^{\dagger} \phi_{3}\right]+\lambda_{11}\left[\phi_{1}^{\dagger} \phi_{1}+\phi_{2}^{\dagger} \phi_{2}+\phi_{3}^{\dagger} \phi_{3}\right]^{2} \\
& +\lambda_{12}\left[\left(\phi_{1}^{\dagger} \phi_{1}\right)^{2}+\left(\phi_{2}^{\dagger} \phi_{2}\right)^{2}+\left(\phi_{3}^{\dagger} \phi_{3}\right)^{2}-\left(\phi_{1}^{\dagger} \phi_{1}\right)\left(\phi_{2}^{\dagger} \phi_{2}\right)-\left(\phi_{2}^{\dagger} \phi_{2}\right)\left(\phi_{3}^{\dagger} \phi_{3}\right)-\left(\phi_{3}^{\dagger} \phi_{3}\right)\left(\phi_{1}^{\dagger} \phi_{1}\right)\right] \\
& +\lambda_{12}^{\prime}\left[\left|\phi_{1}^{\dagger} \phi_{2}\right|^{2}+\left|\phi_{2}^{\dagger} \phi_{3}\right|^{2}+\left|\phi_{3}^{\dagger} \phi_{1}\right|^{2}\right] \\
& +\lambda_{1}\left[\left(\phi_{1}^{\dagger} \phi_{2}\right)\left(\phi_{1}^{\dagger} \phi_{3}\right)+\left(\phi_{2}^{\dagger} \phi_{3}\right)\left(\phi_{2}^{\dagger} \phi_{1}\right)+\left(\phi_{3}^{\dagger} \phi_{1}\right)\left(\phi_{3}^{\dagger} \phi_{2}\right)\right]+\text { h.c. }
\end{aligned}
$$

with real $\mu^{2}, \lambda_{11}, \lambda_{12}, \lambda_{12}^{\prime}$ and complex $\lambda_{1}$. This group is generated by

$$
a=\left(\begin{array}{ccc}
\omega & 0 & 0 \\
0 & \omega^{2} & 0 \\
0 & 0 & 1
\end{array}\right), \quad b=\left(\begin{array}{lll}
0 & 1 & 0 \\
0 & 0 & 1 \\
1 & 0 & 0
\end{array}\right), \quad c=\left(\begin{array}{ccc}
0 & 1 & 0 \\
1 & 0 & 0 \\
0 & 0 & -1
\end{array}\right), \quad \omega=\exp (2 i \pi / 3)
$$

with $a^{3}=1, b^{3}=1$ and $c^{2}=1$.

The possible minima of this potential are:

\footnotetext{
${ }^{9}$ Note that the group $\Delta(27)$, which is the full preimage of the group $Z_{3} \times Z_{3}$ in $\mathrm{SU}(N)$, is not a realizable symmetry of $3 \mathrm{HDM}$ potential since the potential symmetric under $\Delta(27)$ is automatically symmetric under the larger group $\left(Z_{3} \times Z_{3}\right) \rtimes Z_{2}=\Delta(54) / Z_{3}$.
} 
1. Point $\left(0,0, \frac{v}{\sqrt{2}}\right)$ breaks the symmetry of the potential to $Z_{3}$ generated by $a=$ $\left(\omega, \omega^{2}, 1\right)$ and becomes the minimum at

$$
v^{2}=\frac{\mu^{2}}{\lambda_{11}}
$$

provided the following conditions are satisfied:

$$
\begin{array}{ll}
\text { - } & -\lambda_{12}+\lambda_{12}^{\prime}>0 \\
\text { - } & \lambda_{11}+2 \lambda_{12}>0
\end{array}
$$

2. Point $\left(0, \frac{v}{\sqrt{2}}, \frac{v}{\sqrt{2}}\right)$ breaks the symmetry of the potential completely and becomes the minimum only when $\lambda_{1}$ is real, which leads to a larger symmetry of the potential, or when $v_{2}=-v_{3}$. So, we study the conditions for the point $\left(0, \frac{v}{\sqrt{2}},-\frac{v}{\sqrt{2}}\right)$ as the minimum of the potential:

$$
v^{2}=\frac{2 \mu^{2}}{4 \lambda_{11}+\lambda_{12}+\lambda_{12}^{\prime}}
$$

where the following conditions need to be satisfied:

- $\quad-3 \lambda_{12}+\lambda_{12}^{\prime}>0$

- $2 \lambda_{11}+\lambda_{12}>0$

- $\left(-3 \lambda_{12}+\lambda_{12}^{\prime}\right)\left(2 \lambda_{11}+\lambda_{12}\right)>4\left|\lambda_{1}\right|^{2}$

- $\left(-3 \lambda_{12}+\lambda_{12}^{\prime}\right)\left(2 \lambda_{11}+\lambda_{12}\right)^{2}+4\left(\lambda_{1}^{2}+\lambda_{1}^{* 2}\right)\left(2 \lambda_{11}-\lambda_{12}+\lambda_{12}^{\prime}\right)>$

$$
\left(-3 \lambda_{12}+\lambda_{12}^{\prime}\right)\left(2 \lambda_{11}-\lambda_{12}+\lambda_{12}^{\prime}\right)^{2}+8\left|\lambda_{1}\right|^{2}\left(2 \lambda_{11}+\lambda_{12}\right)
$$

3. Point $\left(\frac{v}{\sqrt{2}}, \frac{v}{\sqrt{2}}, \frac{v}{\sqrt{2}}\right)$ breaks the symmetry of the potential to $S_{3}$ (permutation of the three doublets) and becomes the minimum at

$$
v^{2}=\frac{\mu^{2}}{3 \lambda_{11}+\lambda_{12}^{\prime}+\lambda_{1}+\lambda_{1}^{*}}
$$

with the following conditions:

- $\lambda_{11}+\lambda_{12}>2 \operatorname{Re} \lambda_{1}$

- $4\left(\lambda_{11}+\lambda_{12}-2 \operatorname{Re} \lambda_{1}\right)^{2}>\left(4 \operatorname{Re} \lambda_{1}+2 \lambda_{11}-\lambda_{12}+\lambda_{12}^{\prime}\right)^{2}$

- $4\left(\lambda_{11}+\lambda_{12}-2 \operatorname{Re} \lambda_{1}\right)^{3}+\left(4 \operatorname{Re} \lambda_{1}-\lambda_{12}+\lambda_{12}^{\prime}+2 \lambda_{11}\right)^{3}>$

$$
3\left(\lambda_{11}+\lambda_{12}-2 \operatorname{Re} \lambda_{1}\right)\left(4 \operatorname{Re} \lambda_{1}-\lambda_{12}+\lambda_{12}^{\prime}+2 \lambda_{11}\right)^{2}
$$

Higgs mass spectrum for $\left(0,0, \frac{v}{\sqrt{2}}\right)$

The mass spectrum, with

$$
v^{2}=\frac{\mu^{2}}{\lambda_{11}+\lambda_{12}}
$$

has the following form:

$$
\begin{array}{ll}
\mathbf{A}_{3}: & m^{2}=0 \\
\mathbf{H}_{3}^{ \pm}: & m^{2}=0
\end{array}
$$




$$
\begin{aligned}
& \mathbf{H}_{3}: \quad m^{2}=2 \mu^{2} \\
& \mathbf{H}_{2}^{ \pm}: \quad m^{2}=-\mu^{2}+\frac{2 \lambda_{11}-\lambda_{12}}{2} v^{2} \\
& \mathbf{H}_{1}^{ \pm}: \quad m^{2}=-\mu^{2}+\frac{2 \lambda_{11}-\lambda_{12}}{2} v^{2} \\
& \mathbf{H}_{2}^{\prime} \equiv \frac{-H_{2}^{0}+H_{1}^{0}+A_{2}^{0}+A_{1}^{0}}{2}: \quad m^{2}=-\mu^{2}+\frac{1}{2}\left(2 \lambda_{11}-\lambda_{12}+\lambda_{12}^{\prime}-\operatorname{Re} \lambda_{1}-\operatorname{Im} \lambda_{1}\right) v^{2} \\
& \mathbf{H}_{1}^{\prime} \equiv \frac{H_{2}^{0}-H_{1}^{0}+A_{2}^{0}+A_{1}^{0}}{2}: \quad m^{2}=-\mu^{2}+\frac{1}{2}\left(2 \lambda_{11}-\lambda_{12}+\lambda_{12}^{\prime}-\operatorname{Re} \lambda_{1}+\operatorname{Im} \lambda_{1}\right) v^{2} \\
& \mathbf{A}_{2}^{\prime} \equiv \frac{H_{2}^{0}+H_{1}^{0}-A_{2}^{0}+A_{1}^{0}}{2}: \quad m^{2}=-\mu^{2}+\frac{1}{2}\left(2 \lambda_{11}-\lambda_{12}+\lambda_{12}^{\prime}+\operatorname{Re} \lambda_{1}+\operatorname{Im} \lambda_{1}\right) v^{2} \\
& \mathbf{A}_{1}^{\prime} \equiv \frac{H_{2}^{0}+H_{1}^{0}+A_{2}^{0}-A_{1}^{0}}{2}: \quad m^{2}=-\mu^{2}+\frac{1}{2}\left(2 \lambda_{11}-\lambda_{12}+\lambda_{12}^{\prime}+\operatorname{Re} \lambda_{1}-\operatorname{Im} \lambda_{1}\right) v^{2}
\end{aligned}
$$

The lightest neutral field from the first or the second doublet, stabilised by the remaining $Z_{3}$ symmetry, is a viable DM candidate.

\section{6 $\Sigma(36) \simeq\left(\mathrm{Z}_{3} \times \mathrm{Z}_{3}\right) \rtimes \mathrm{Z}_{4}$ symmetric $3 \mathrm{HDM}$ potential}

A $\Sigma(36)$-symmetric $3 \mathrm{HDM}$ potential has the following form:

$$
\begin{aligned}
V_{\Sigma(36)}= & -\mu^{2}\left(\phi_{1}^{\dagger} \phi_{1}+\phi_{2}^{\dagger} \phi_{2}+\phi_{3}^{\dagger} \phi_{3}\right)+\lambda_{11}\left(\phi_{1}^{\dagger} \phi_{1}+\phi_{2}^{\dagger} \phi_{2}+\phi_{3}^{\dagger} \phi_{3}\right)^{2} \\
& +\lambda_{12}^{\prime}\left(\left|\phi_{1}^{\dagger} \phi_{2}-\phi_{2}^{\dagger} \phi_{3}\right|^{2}+\left|\phi_{2}^{\dagger} \phi_{3}-\phi_{3}^{\dagger} \phi_{1}\right|^{2}+\left|\phi_{3}^{\dagger} \phi_{1}-\phi_{1}^{\dagger} \phi_{2}\right|^{2}\right) .
\end{aligned}
$$

This group is generated by arbitrary permutations of the three doublets, and by:

$$
a=\left(\begin{array}{ccc}
1 & 0 & 0 \\
0 & \omega & 0 \\
0 & 0 & \omega^{2}
\end{array}\right), \quad d=\frac{1}{\omega^{2}-\omega}\left(\begin{array}{ccc}
1 & 1 & 1 \\
1 & \omega^{2} & \omega \\
1 & \omega & \omega^{2}
\end{array}\right), \quad \omega=\exp (2 i \pi / 3)
$$

with $a^{3}=1$ and $d^{4}=1$.

The possible minima of this potential are:

1. Point $\left(0,0, \frac{v}{\sqrt{2}}\right)$ breaks the symmetry of the potential to $Z_{3}$ generated by $a=$ $\left(\omega, \omega^{2}, 1\right)$ and becomes the minimum at

$$
v^{2}=\frac{\mu^{2}}{\lambda_{11}}
$$

provided the following conditions are satisfied:

$$
\begin{array}{ll}
\text { - } & \lambda_{12}^{\prime}>0 \\
\text { - } & \mu^{2}>0
\end{array}
$$

The last condition is already required for the positivity of mass eigenstates at point $\left(0,0, \frac{v}{\sqrt{2}}\right)$. 
2. Point $\left(0, \frac{v}{\sqrt{2}}, \frac{v}{\sqrt{2}}\right)$ breaks the symmetry of the potential completely and becomes the minimum at

$$
v^{2}=\frac{\mu^{2}}{2 \lambda_{11}+\lambda_{12}^{\prime}}
$$

when the following conditions are satisfied:

- $12 \lambda_{11}>\lambda_{12}^{\prime}>0$

- $8\left(\lambda_{11}\right)^{2}-\frac{27}{4} \lambda_{11} \lambda_{12}^{\prime}-2\left(\lambda_{12}^{\prime}\right)^{2}>0$

3. Point $\left(\frac{v}{\sqrt{2}}, \frac{v}{\sqrt{2}}, \frac{v}{\sqrt{2}}\right)$ breaks the symmetry of the potential completely and becomes the minimum at

$$
v^{2}=\frac{\mu^{2}}{3 \lambda_{11}}
$$

with the following conditions:

- $\lambda_{11}+2 \lambda_{12}^{\prime}>0$

- $4\left(\lambda_{11}+2 \lambda_{12}^{\prime}\right)^{2}>\left(2 \lambda_{11}-\lambda_{12}^{\prime}\right)^{2}$

- $4\left(\lambda_{11}+2 \lambda_{12}^{\prime}\right)^{3}+\left(2 \lambda_{11}-\lambda_{12}^{\prime}\right)^{3}>3\left(\lambda_{11}+2 \lambda_{12}^{\prime}\right)\left(2 \lambda_{11}-\lambda_{12}^{\prime}\right)^{2}$

Higgs mass spectrum for $\left(0,0, \frac{v}{\sqrt{2}}\right)$

Expanding the potential around the vacuum point $\left(0,0, \frac{v}{\sqrt{2}}\right)$, with

$$
v^{2}=\frac{\mu^{2}}{\lambda_{11}}
$$

the mass spectrum appears as follows:

$$
\begin{array}{ll}
\mathbf{A}_{3}: & m^{2}=0 \\
\mathbf{H}_{3}^{ \pm}: & m^{2}=0 \\
\mathbf{H}_{3}: & m^{2}=2 \mu^{2} \\
\mathbf{H}_{2}^{ \pm}: & m^{2}=-\mu^{2}+\lambda_{11} v^{2} \\
\mathbf{H}_{1}^{ \pm}: & m^{2}=-\mu^{2}+\lambda_{11} v^{2} \\
\mathbf{H}_{2} \equiv \frac{H_{2}^{0}-H_{1}^{0}}{\sqrt{2}}: \quad m^{2}=-\mu^{2}+\frac{1}{2}\left(2 \lambda_{11}+3 \lambda_{12}^{\prime}\right) v^{2} \\
\mathbf{H}_{1} \equiv \frac{H_{2}^{0}+H_{1}^{0}}{\sqrt{2}}: \quad m^{2}=-\mu^{2}+\frac{1}{2}\left(2 \lambda_{11}+\lambda_{12}^{\prime}\right) v^{2} \\
\mathbf{A}_{2} \equiv \frac{A_{2}^{0}-A_{1}^{0}}{\sqrt{2}}: \quad m^{2}=-\mu^{2}+\frac{1}{2}\left(2 \lambda_{11}+\lambda_{12}^{\prime}\right) v^{2} \\
\mathbf{A}_{1} \equiv \frac{A_{2}^{0}+A_{1}^{0}}{\sqrt{2}}: \quad m^{2}=-\mu^{2}+\frac{1}{2}\left(2 \lambda_{11}+3 \lambda_{12}^{\prime}\right) v^{2}
\end{array}
$$

It is interesting to note that the minimisation condition (4.34) results in $m_{\mathbf{H}_{1}^{ \pm}}^{2}$ and $m_{\mathbf{H}_{2}^{ \pm}}^{2}$ vanishing at tree-level. However, this is accidental (there is no symmetry reason for their vanishing) and so we expect them to acquire small masses at higher order.

The viable DM candidate in this case is the lightest neutral field from the first or the second doublet, stabilised by the remaining $Z_{3}$ symmetry. 


\section{Analysis of $6 \mathrm{HDMs}$}

We define each doublet $\phi_{i}$ as a doublet of doublets

$$
\phi_{i}=\left(\begin{array}{c}
H_{u}^{i} \\
H_{d}^{i}
\end{array}\right)
$$

where $H_{u}^{i}$ and $H_{d}^{i}$ are defined in the following way:

$$
H_{u}^{i}=\left(\begin{array}{c}
H_{i u}^{+} \\
\frac{H_{i u}^{0}+i A_{i u}^{0}}{\sqrt{2}}
\end{array}\right), \quad H_{d}^{i}=\left(\begin{array}{c}
H_{i d}^{+} \\
\frac{H_{i d}^{0}+i A_{i d}^{0}}{\sqrt{2}}
\end{array}\right)
$$

We add the following MSSM-inspired term to avoid extra massless particles:

$$
\mu^{\prime \prime 2}\left(H_{1 u}^{\dagger} H_{1 d}+H_{2 u}^{\dagger} H_{2 d}+H_{3 u}^{\dagger} H_{3 d}\right)+\text { h.c. }
$$

Note that these terms do not break the symmetry of the potential, since they only mix the intra-doublet fields $H_{i u}$ and $H_{i d}$ and not the doublets $\phi_{i}$.

Extending the $\left(0,0, \frac{v}{\sqrt{2}}\right)$ minimum to 6 Higgs doublets results in a VEV alignment of the form $\left(0,0,0,0, \frac{v}{\sqrt{2}}, \frac{v}{\sqrt{2}}\right)$, with two active doublets:

$$
\left\langle H_{3 u}\right\rangle=\left\langle H_{3 d}\right\rangle=v
$$

and four inert doublets;

$$
\left\langle H_{1 u}\right\rangle=\left\langle H_{1 d}\right\rangle=\left\langle H_{2 u}\right\rangle=\left\langle H_{2 d}\right\rangle=0 .
$$

In the following sections we present the mass spectrum in each case.

\section{$5.1 \quad \mathrm{U}(1) \times \mathrm{U}(1)$ symmetric 6 HDM potential}

The mass spectrum of the $\mathrm{U}(1) \times \mathrm{U}(1)$ symmetric 6 HDM potential around the minimum point $\left(0,0,0,0, \frac{v}{\sqrt{2}}, \frac{v}{\sqrt{2}}\right)$ with

$$
v^{2}=\frac{\mu_{3}^{2}-\mu^{\prime 2}}{2 \lambda_{33}}
$$

has the following form:

$$
\begin{array}{ll}
\mathbf{G}_{3}=\frac{A_{3 u}^{0}+A_{3 d}^{0}}{\sqrt{2}}: & m^{2}=0 \\
\mathbf{G}_{3}^{ \pm}=\frac{H_{3 u}^{ \pm}+H_{3 d}^{ \pm}}{\sqrt{2}}: & m^{2}=0 \\
\mathbf{h}_{3}=\frac{H_{3 u}^{0}+H_{3 d}^{0}}{\sqrt{2}}: & m^{2}=2 \mu_{3}^{2}-2 \mu^{\prime 2} \\
\mathbf{H}_{3}=\frac{H_{3 u}^{0}-H_{3 d}^{0}}{\sqrt{2}}: & m^{2}=-2 \mu^{\prime 2} \\
\mathbf{A}_{3}=\frac{A_{3 u}^{0}-A_{3 d}^{0}}{\sqrt{2}}: & m^{2}=-2 \mu^{\prime 2} \\
\mathbf{H}_{3}^{ \pm}=\frac{H_{3 u}^{ \pm}-H_{3 d}^{ \pm}}{\sqrt{2}}: & m^{2}=-2 \mu^{\prime 2}
\end{array}
$$




$$
\begin{array}{ll}
\mathbf{h}_{2}=\frac{H_{2 u}^{0}+H_{2 d}^{0}}{\sqrt{2}}: & m^{2}=-\mu_{2}^{2}+\mu^{\prime 2}+\left(\lambda_{23}+\lambda_{23}^{\prime}\right) v^{2} \\
\mathbf{G}_{2}=\frac{A_{2 u}^{0}+A_{2 d}^{0}}{\sqrt{2}}: & m^{2}=-\mu_{2}^{2}+\mu^{\prime 2}+\left(\lambda_{23}+\lambda_{23}^{\prime}\right) v^{2} \\
\mathbf{G}_{2}^{ \pm}=\frac{H_{2 u}^{ \pm}+H_{2 d}^{ \pm}}{\sqrt{2}}: & m^{2}=-\mu_{2}^{2}+\mu^{\prime 2}+\lambda_{23} v^{2} \\
\mathbf{H}_{2}=\frac{H_{2 u}^{0}-H_{2 d}^{0}}{\sqrt{2}}: & m^{2}=-\mu_{2}^{2}-\mu^{\prime 2}+\lambda_{23} v^{2} \\
\mathbf{A}_{2}=\frac{A_{2 u}^{0}-A_{2 d}^{0}}{\sqrt{2}}: & m^{2}=-\mu_{2}^{2}-\mu^{\prime 2}+\lambda_{23} v^{2} \\
\mathbf{H}_{2}^{ \pm}=\frac{H_{2 u}^{ \pm}-H_{2 d}^{ \pm}}{\sqrt{2}}: & m^{2}=-\mu_{2}^{2}-\mu^{\prime 2}+\lambda_{23} v^{2} \\
\mathbf{h}_{1}=\frac{H_{1 u}^{0}+H_{1 d}^{0}}{\sqrt{2}}: & m^{2}=-\mu_{1}^{2}+\mu^{\prime 2}+\left(\lambda_{31}+\lambda_{31}^{\prime}\right) v^{2} \\
\mathbf{G}_{1}=\frac{A_{1 u}^{0}+A_{1 d}^{0}}{\sqrt{2}}: & m^{2}=-\mu_{1}^{2}+\mu^{\prime 2}+\left(\lambda_{31}+\lambda_{31}^{\prime}\right) v^{2} \\
\mathbf{G}_{1}^{ \pm}=\frac{H_{1 u}^{ \pm}+H_{1 d}^{ \pm}}{\sqrt{2}}: & m^{2}=-\mu_{1}^{2}+\mu^{\prime 2}+\lambda_{31} v^{2} \\
\mathbf{H}_{1}=\frac{H_{1 u}^{0}-H_{1 d}^{0}}{\sqrt{2}}: & m^{2}=-\mu_{1}^{2}-\mu^{\prime 2}+\lambda_{31} v^{2} \\
\mathbf{A}_{1}=\frac{A_{1 u}^{0}-A_{1 d}^{0}}{\sqrt{2}}: & m^{2}=-\mu_{1}^{2}-\mu^{\prime 2}+\lambda_{31} v^{2} \\
\mathbf{H}_{1}^{ \pm}=\frac{H_{1 u}^{ \pm}-H_{1 d}^{ \pm}}{\sqrt{2}}: & m^{2}=-\mu_{1}^{2}-\mu^{\prime 2}+\lambda_{31} v^{2} \\
&
\end{array}
$$

\section{$5.2 \mathrm{U}(1)$ symmetric 6 HDM potential}

The mass spectrum of the $\mathrm{U}(1)$ symmetric $6 \mathrm{HDM}$ potential around the minimum point $\left(0,0,0,0, \frac{v}{\sqrt{2}}, \frac{v}{\sqrt{2}}\right)$ with

$$
v^{2}=\frac{\mu_{3}^{2}-\mu^{\prime 2}}{2 \lambda_{33}}
$$

has the following form:

$$
\begin{array}{ll}
\mathbf{G}_{3}=\frac{A_{3 u}^{0}+A_{3 d}^{0}}{\sqrt{2}}: & m^{2}=0 \\
\mathbf{G}_{3}^{ \pm}=\frac{H_{3 u}^{ \pm}+H_{3 d}^{ \pm}}{\sqrt{2}}: & m^{2}=0 \\
\mathbf{h}_{3}=\frac{H_{3 u}^{0}+H_{3 d}^{0}}{\sqrt{2}}: & m^{2}=2 \mu_{3}^{2}-2 \mu^{\prime 2} \\
\mathbf{H}_{3}=\frac{H_{3 u}^{0}-H_{3 d}^{0}}{\sqrt{2}}: & m^{2}=-2 \mu^{\prime 2} \\
\mathbf{A}_{3}=\frac{A_{3 u}^{0}-A_{3 d}^{0}}{\sqrt{2}}: & m^{2}=-2 \mu^{\prime 2} \\
\mathbf{H}_{3}^{ \pm}=\frac{H_{3 u}^{ \pm}-H_{3 d}^{ \pm}}{\sqrt{2}}: & m^{2}=-2 \mu^{\prime 2} \\
\mathbf{H}_{2}^{ \pm}=\frac{H_{2 u}^{ \pm}-H_{2 d}^{ \pm}}{\sqrt{2}}: & m^{2}=-\mu_{2}^{2}-\mu^{\prime 2}+\lambda_{23} v^{2} \\
\mathbf{G}_{2}^{ \pm}=\frac{H_{2 u}^{ \pm}+H_{2 d}^{ \pm}}{\sqrt{2}}: & m^{2}=-\mu_{2}^{2}+\mu^{\prime 2}+\lambda_{23} v^{2}
\end{array}
$$




$$
\begin{aligned}
& \mathbf{H}_{1}^{ \pm}=\frac{H_{1 u}^{ \pm}-H_{1 d}^{ \pm}}{\sqrt{2}}: \quad m^{2}=-\mu_{1}^{2}-\mu^{\prime 2}+\lambda_{31} v^{2} \\
& \mathbf{G}_{1}^{ \pm}=\frac{H_{1 u}^{ \pm}+H_{1 d}^{ \pm}}{\sqrt{2}}: \quad m^{2}=-\mu_{1}^{2}+\mu^{\prime 2}+\lambda_{31} v^{2} \\
& \mathbf{A}_{2}=\frac{A_{2 u}^{0}-A_{2 d}^{0}}{\sqrt{2}}: \quad m^{2}=-\mu_{2}^{2}-\mu^{\prime 2}+\lambda_{23} v^{2} \\
& \mathbf{A}_{1}=\frac{A_{1 u}^{0}-A_{1 d}^{0}}{\sqrt{2}}: \quad m^{2}=-\mu_{1}^{2}-\mu^{\prime 2}+\lambda_{31} v^{2} \\
& \mathbf{G}_{2}^{\prime}=\frac{-a A_{2 u}^{0}-a A_{2 d}^{0}+A_{1 u}^{0}+A_{1 d}^{0}}{\sqrt{2+2 a^{2}}}: \quad m^{2}=X-\sqrt{Y} \\
& \mathbf{G}_{1}^{\prime}=\frac{-b A_{2 u}^{0}-b A_{2 d}^{0}+A_{1 u}^{0}+A_{1 d}^{0}}{\sqrt{2+2 b^{2}}}: \quad m^{2}=X+\sqrt{Y} \\
& \mathbf{H}_{2}=\frac{H_{2 u}^{0}-H_{2 d}^{0}}{\sqrt{2}}: \quad m^{2}=-\mu_{2}^{2}-\mu^{\prime 2}+\lambda_{23} v^{2} \\
& \mathbf{H}_{1}=\frac{H_{1 u}^{0}-H_{1 d}^{0}}{\sqrt{2}}: \quad m^{2}=-\mu_{1}^{2}-\mu^{\prime 2}+\lambda_{31} v^{2} \\
& \mathbf{h}_{2}^{\prime}=\frac{a H_{2 u}^{0}+a H_{2 d}^{0}+H_{1 u}^{0}+H_{1 d}^{0}}{\sqrt{2+2 a^{2}}}: \quad m^{2}=X-\sqrt{Y} \\
& \mathbf{h}_{1}^{\prime}=\frac{b H_{2 u}^{0}+b H_{2 d}^{0}+H_{1 u}^{0}+H_{1 d}^{0}}{\sqrt{2+2 b^{2}}}: \quad m^{2}=X+\sqrt{Y} \\
& \text { where } \quad X=\frac{1}{2}\left[2 \mu^{\prime 2}-\mu_{1}^{2}-\mu_{2}^{2}+\left(\lambda_{23}+\lambda_{31}+\lambda_{23}^{\prime}+\lambda_{31}^{\prime}\right) v^{2}\right] \\
& Y=\left(\lambda_{1} v^{2}\right)^{2}+\frac{1}{4}\left[\mu_{1}^{2}-\mu_{2}^{2}+\left(\lambda_{23}-\lambda_{31}+\lambda_{23}^{\prime}-\lambda_{31}^{\prime}\right) v^{2}\right]^{2} \\
& a=\frac{1}{\lambda_{1} v^{2}}\left[\mu_{1}^{2}-\mu_{2}^{2}+\left(\lambda_{23}-\lambda_{31}+\lambda_{23}^{\prime}-\lambda_{31}^{\prime}\right) v^{2}-\sqrt{Y}\right] \\
& b=\frac{1}{\lambda_{1} v^{2}}\left[\mu_{1}^{2}-\mu_{2}^{2}+\left(\lambda_{23}-\lambda_{31}+\lambda_{23}^{\prime}-\lambda_{31}^{\prime}\right) v^{2}+\sqrt{Y}\right]
\end{aligned}
$$

\section{3 $\mathrm{U}(1) \times \mathrm{Z}_{2}$ symmetric $6 \mathrm{HDM}$ potential}

The mass spectrum of the $\mathrm{U}(1) \times Z_{2}$ symmetric $6 \mathrm{HDM}$ potential around the minimum point $\left(0,0,0,0, \frac{v}{\sqrt{2}}, \frac{v}{\sqrt{2}}\right)$ with

$$
v^{2}=\frac{\mu_{3}^{2}-\mu^{\prime 2}}{2 \lambda_{33}}
$$

has the following form:

$$
\begin{array}{ll}
\mathbf{G}_{3}=\frac{A_{3 u}^{0}+A_{3 d}^{0}}{\sqrt{2}}: & m^{2}=0 \\
\mathbf{G}_{3}^{ \pm}=\frac{H_{3 u}^{ \pm}+H_{3 d}^{ \pm}}{\sqrt{2}}: & m^{2}=0 \\
\mathbf{h}_{3}=\frac{H_{3 u}^{0}+H_{3 d}^{0}}{\sqrt{2}}: & m^{2}=2 \mu_{3}^{2}-2 \mu^{\prime 2} \\
\mathbf{H}_{3}=\frac{H_{3 u}^{0}-H_{3 d}^{0}}{\sqrt{2}}: & m^{2}=-2 \mu^{\prime 2} \\
\mathbf{A}_{3}=\frac{A_{3 u}^{0}-A_{3 d}^{0}}{\sqrt{2}}: & m^{2}=-2 \mu^{\prime 2} \\
\mathbf{H}_{3}^{ \pm}=\frac{H_{3 u}^{ \pm}-H_{3 d}^{ \pm}}{\sqrt{2}}: & m^{2}=-2 \mu^{\prime 2} \\
\mathbf{h}_{2}=\frac{H_{2 u}^{0}+H_{2 d}^{0}}{\sqrt{2}}: & m^{2}=-\mu_{2}^{2}+\mu^{\prime 2}+\left(\lambda_{23}+\lambda_{23}^{\prime}+\lambda_{1}\right) v^{2}
\end{array}
$$




$$
\begin{array}{ll}
\mathbf{G}_{2}=\frac{A_{2 u}^{0}+A_{2 d}^{0}}{\sqrt{2}}: & m^{2}=-\mu_{2}^{2}+\mu^{\prime 2}+\left(\lambda_{23}+\lambda_{23}^{\prime}-\lambda_{1}\right) v^{2} \\
\mathbf{G}_{2}^{ \pm}=\frac{H_{2 u}^{ \pm}+H_{2 d}^{ \pm}}{\sqrt{2}}: & m^{2}=-\mu_{2}^{2}+\mu^{\prime 2}+\lambda_{23} v^{2} \\
\mathbf{H}_{2}=\frac{H_{2 u}^{0}-H_{2 d}^{0}}{\sqrt{2}}: & m^{2}=-\mu_{2}^{2}-\mu^{\prime 2}+\lambda_{23} v^{2} \\
\mathbf{A}_{2}=\frac{A_{2 u}^{0}-A_{2 d}^{0}}{\sqrt{2}}: & m^{2}=-\mu_{2}^{2}-\mu^{\prime 2}+\lambda_{23} v^{2} \\
\mathbf{H}_{2}^{ \pm}=\frac{H_{2 u}^{ \pm}-H_{2 d}^{ \pm}}{\sqrt{2}}: & m^{2}=-\mu_{2}^{2}-\mu^{\prime 2}+\lambda_{23} v^{2} \\
\mathbf{h}_{1}=\frac{H_{1 u}^{0}+H_{1 d}^{0}}{\sqrt{2}}: & m^{2}=-\mu_{1}^{2}+\mu^{\prime 2}+\left(\lambda_{31}+\lambda_{31}^{\prime}\right) v^{2} \\
\mathbf{G}_{1}=\frac{A_{1 u}^{0}+A_{1 d}^{0}}{\sqrt{2}}: & m^{2}=-\mu_{1}^{2}+\mu^{\prime 2}+\left(\lambda_{31}+\lambda_{31}^{\prime}\right) v^{2} \\
\mathbf{G}_{1}^{ \pm}=\frac{H_{1 u}^{ \pm}+H_{1 d}^{ \pm}}{\sqrt{2}}: & m^{2}=-\mu_{1}^{2}+\mu^{\prime 2}+\lambda_{31} v^{2} \\
\mathbf{H}_{1}=\frac{H_{1 u}^{0}-H_{1 d}^{0}}{\sqrt{2}}: & m^{2}=-\mu_{1}^{2}-\mu^{\prime 2}+\lambda_{31} v^{2} \\
\mathbf{A}_{1}=\frac{A_{1 u}^{0}-A_{1 d}^{0}}{\sqrt{2}}: & m^{2}=-\mu_{1}^{2}-\mu^{\prime 2}+\lambda_{31} v^{2} \\
\mathbf{H}_{1}^{ \pm}=\frac{H_{1 u}^{ \pm}-H_{1 d}^{ \pm}}{\sqrt{2}}: & m^{2}=-\mu_{1}^{2}-\mu^{\prime 2}+\lambda_{31} v^{2} \\
&
\end{array}
$$

\section{$5.4 \mathrm{Z}_{2}$ symmetric $6 \mathrm{HDM}$ potential}

The mass spectrum of the $Z_{2}$ symmetric $6 \mathrm{HDM}$ potential around the minimum point $\left(0,0,0,0, \frac{v}{\sqrt{2}}, \frac{v}{\sqrt{2}}\right)$ with

$$
v^{2}=\frac{\mu_{3}^{2}-\mu^{\prime 2}}{2 \lambda_{33}}
$$

has the following form:

$$
\begin{aligned}
& \mathbf{G}_{3}=\frac{A_{3 u}^{0}+A_{3 d}^{0}}{\sqrt{2}}: \quad m^{2}=0 \\
& \mathbf{G}_{3}^{ \pm}=\frac{H_{3 u}^{ \pm}+H_{3 d}^{ \pm}}{\sqrt{2}}: \quad m^{2}=0 \\
& \mathbf{h}_{3}=\frac{H_{3 u}^{0}+H_{3 d}^{0}}{\sqrt{2}}: \quad m^{2}=2 \mu_{3}^{2}-2 \mu^{\prime 2} \\
& \mathbf{H}_{3}=\frac{H_{3 u}^{0}-H_{3 d}^{0}}{\sqrt{2}}: \quad m^{2}=-2 \mu^{\prime 2} \\
& \mathbf{A}_{3}=\frac{A_{3 u}^{0}-A_{3 d}^{0}}{\sqrt{2}}: \quad m^{2}=-2 \mu^{\prime 2} \\
& \mathbf{H}_{3}^{ \pm}=\frac{H_{3 u}^{ \pm}-H_{3 d}^{ \pm}}{\sqrt{2}}: \quad m^{2}=-2 \mu^{\prime 2} \\
& \mathbf{h}_{2}^{\prime}=\frac{X H_{2 u}^{0}-X H_{2 d}^{0}-H_{1 u}^{0}+H_{1 d}^{0}}{\sqrt{2+2 X^{2}}:} \quad m^{2}=\alpha_{1}+\alpha_{2}-\beta_{1}-\beta_{2}-\sqrt{\Delta} \\
& \mathbf{H}_{2}^{\prime}=\frac{-Y H_{2 u}^{0}+Y H_{2 d}^{0}-H_{1 u}^{0}+H_{1 d}^{0}}{\sqrt{2+2 Y^{2}}}: \quad m^{2}=\alpha_{1}+\alpha_{2}-\beta_{1}-\beta_{2}+\sqrt{\Delta} \\
& \mathbf{h}_{1}^{\prime}=\frac{-W H_{2 u}^{0}-W H_{2 d}^{0}+H_{1 u}^{0}+H_{1 d}^{0}}{\sqrt{2+2 W^{2}}:} \quad m^{2}=\alpha_{1}+\alpha_{2}+\beta_{1}+\beta_{2}-\sqrt{\Delta}
\end{aligned}
$$




$$
\begin{aligned}
& \mathbf{H}_{1}^{\prime}=\frac{Z H_{2 u}^{0}+Z H_{2 d}^{0}+H_{1 u}^{0}+H_{1 d}^{0}}{\sqrt{2+2 Z^{2}}}: \quad m^{2}=\alpha_{1}+\alpha_{2}+\beta_{1}+\beta_{2}+\sqrt{\Delta} \\
& \text { where } \quad \alpha_{1}=-\frac{\mu_{2}^{2}}{2}+\frac{1}{4}\left(2 \lambda_{23}+\lambda_{23}^{\prime}+2 \lambda_{2}\right) v^{2} \\
& \alpha_{2}=-\frac{\mu_{1}^{2}}{2}+\frac{1}{4}\left(2 \lambda_{31}+\lambda_{31}^{\prime}+2 \lambda_{3}\right) v^{2} \\
& \beta_{1}=\frac{\mu^{\prime 2}}{2}+\frac{1}{4}\left(\lambda_{23}^{\prime}+2 \lambda_{2}\right) v^{2} \\
& \beta_{2}=\frac{\mu^{\prime 2}}{2}+\frac{1}{4}\left(\lambda_{31}^{\prime}+2 \lambda_{3}\right) v^{2} \\
& \gamma=-\frac{\mu_{12}^{2}}{2} \\
& \mathbf{G}_{2}^{\prime}=\frac{X^{\prime} A_{2 u}^{0}-X^{\prime} A_{2 d}^{0}-A_{1 u}^{0}+A_{1 d}^{0}}{\sqrt{2+2 X^{\prime 2}}}: \quad m^{2}=\alpha_{1}^{\prime}+\alpha_{2}^{\prime}-\beta_{1}^{\prime}-\beta_{2}^{\prime}-\sqrt{\Delta^{\prime}} \\
& \mathbf{A}_{2}^{\prime}=\frac{-Y^{\prime} A_{2 u}^{0}-Y^{\prime} A_{2 d}^{0}-A_{1 u}^{0}+A_{1 d}^{0}}{\sqrt{2+2 Y^{\prime 2}}}: \quad m^{2}=\alpha_{1}^{\prime}+\alpha_{2}^{\prime}-\beta_{1}^{\prime}-\beta_{2}^{\prime}+\sqrt{\Delta^{\prime}} \\
& \mathbf{G}_{1}^{\prime}=\frac{-W^{\prime} A_{2 u}^{0}-W^{\prime} A_{2 d}^{0}+A_{1 u}^{0}+A_{1 d}^{0}}{\sqrt{2+2 W^{\prime 2}}}: \quad m^{2}=\alpha_{1}^{\prime}+\alpha_{2}^{\prime}+\beta_{1}^{\prime}+\beta_{2}^{\prime}-\sqrt{\Delta^{\prime}} \\
& \mathbf{A}_{1}^{\prime}=\frac{Z^{\prime} A_{2 u}^{0}+Z^{\prime} A_{2 d}^{0}+A_{1 u}^{0}+A_{1 d}^{0}}{\sqrt{2+2 Z^{\prime 2}}}: \quad m^{2}=\alpha_{1}^{\prime}+\alpha_{2}^{\prime}+\beta_{1}^{\prime}+\beta_{2}^{\prime}+\sqrt{\Delta^{\prime}} \\
& \text { where } \quad \alpha_{1}^{\prime}=-\frac{\mu_{2}^{2}}{2}+\frac{1}{4}\left(2 \lambda_{23}+\lambda_{23}^{\prime}-2 \lambda_{2}\right) v^{2} \\
& \alpha_{2}^{\prime}=-\frac{\mu_{1}^{2}}{2}+\frac{1}{4}\left(2 \lambda_{31}+\lambda_{31}^{\prime}-2 \lambda_{3}\right) v^{2} \\
& \beta_{1}^{\prime}=\frac{\mu^{\prime 2}}{2}+\frac{1}{4}\left(\lambda_{23}^{\prime}-2 \lambda_{2}\right) v^{2} \\
& \beta_{2}^{\prime}=\frac{\mu^{\prime 2}}{2}+\frac{1}{4}\left(\lambda_{31}^{\prime}-2 \lambda_{3}\right) v^{2} \\
& \gamma^{\prime}=-\frac{\mu_{12}^{2}}{2} \\
& \mathbf{H}_{2}^{\prime \pm}=\frac{X^{\prime \prime} H_{2 u}^{ \pm}-X^{\prime \prime} H_{2 d}^{ \pm}-H_{1 u}^{ \pm}+H_{1 d}^{ \pm}}{\sqrt{2+2 X^{\prime \prime 2}}}: \quad m^{2}=\alpha_{1}^{\prime \prime}+\alpha_{2}^{\prime \prime}-\beta_{1}^{\prime \prime}-\beta_{2}^{\prime \prime}-\sqrt{\Delta^{\prime \prime}} \\
& \mathbf{G}_{2}^{\prime \pm}=\frac{-Y^{\prime \prime} H_{2 u}^{ \pm}+Y^{\prime \prime} H_{2 d}^{ \pm}-H_{1 u}^{ \pm}+H_{1 d}^{ \pm}}{\sqrt{2+2 Y^{\prime \prime 2}}}: \quad m^{2}=\alpha_{1}^{\prime \prime}+\alpha_{2}^{\prime \prime}-\beta_{1}^{\prime \prime}-\beta_{2}^{\prime \prime}+\sqrt{\Delta^{\prime \prime}} \\
& \mathbf{H}_{1}^{\prime \pm}=\frac{-W^{\prime \prime} H_{2 u}^{ \pm}-W^{\prime \prime} H_{2 d}^{ \pm}+H_{1 u}^{ \pm}+H_{1 d}^{ \pm}}{\sqrt{2+2 W^{\prime \prime 2}}}: \quad m^{2}=\alpha_{1}^{\prime \prime}+\alpha_{2}^{\prime \prime}+\beta_{1}^{\prime \prime}+\beta_{2}^{\prime \prime}-\sqrt{\Delta^{\prime \prime}} \\
& \mathbf{G}_{1}^{\prime \pm}=\frac{Z^{\prime \prime} H_{2 u}^{ \pm}+Z^{\prime \prime} H_{2 d}^{ \pm}+H_{1 u}^{ \pm}+H_{1 d}^{ \pm}}{\sqrt{2+2 Z^{\prime \prime 2}}}: \quad m^{2}=\alpha_{1}^{\prime \prime}+\alpha_{2}^{\prime \prime}+\beta_{1}^{\prime \prime}+\beta_{2}^{\prime \prime}+\sqrt{\Delta^{\prime \prime}} \\
& \text { where } \quad \alpha_{1}^{\prime \prime}=-\mu_{2}^{2}+\lambda_{23} v^{2} \\
& \alpha_{2}^{\prime \prime}=-\mu_{1}^{2}+\lambda_{31} v^{2} \\
& \beta_{1}^{\prime \prime}=\beta_{2}^{\prime \prime}=\mu^{\prime 2}, \quad \gamma^{\prime \prime}=-\mu_{12}^{2} \\
& \text { and } \quad X=\frac{1}{2 \gamma}\left[-\alpha_{1}+\alpha_{2}+\beta_{1}-\beta_{2}+\sqrt{\Delta}\right] \\
& Y=\frac{1}{2 \gamma}\left[\alpha_{1}-\alpha_{2}-\beta_{1}+\beta_{2}+\sqrt{\Delta}\right]
\end{aligned}
$$




$$
\begin{aligned}
W & =\frac{1}{2 \gamma}\left[-\alpha_{1}+\alpha_{2}-\beta_{1}+\beta_{2}+\sqrt{\Delta}\right] \\
Z & =\frac{1}{2 \gamma}\left[\alpha_{1}-\alpha_{2}+\beta_{1}-\beta_{2}+\sqrt{\Delta}\right] \\
\Delta & =\left(\alpha_{1}-\alpha_{2}-\beta_{1}+\beta_{2}\right)^{2}+4 \gamma^{2}
\end{aligned}
$$

and $X^{\prime}, Y^{\prime}, W^{\prime}, Z^{\prime}$ and $X^{\prime \prime}, Y^{\prime \prime}, W^{\prime \prime}, Z^{\prime \prime}$ are defined in the same way as $X, Y, W, Z$ with the corresponding primed and double-primed $\alpha, \beta$ and $\gamma$ s.

\section{$5.5 \mathrm{Z}_{3}$ symmetric $6 \mathrm{HDM}$ potential}

The mass spectrum of the $Z_{3}$ symmetric $6 \mathrm{HDM}$ potential around the minimum point $\left(0,0,0,0, \frac{v}{\sqrt{2}}, \frac{v}{\sqrt{2}}\right)$ with

$$
v^{2}=\frac{\mu_{3}^{2}-\mu^{\prime 2}}{2 \lambda_{33}}
$$

is the same as the $\mathrm{U}(1)$-symmetric case with a slight difference (replace $\lambda_{1}$ by $\lambda_{2}$ ).

\section{$5.6 \quad \mathrm{Z}_{4}$ symmetric $6 \mathrm{HDM}$ potential}

The mass spectrum of the $Z_{4}$ symmetric $6 \mathrm{HDM}$ potential around the minimum point $\left(0,0,0,0, \frac{v}{\sqrt{2}}, \frac{v}{\sqrt{2}}\right)$ with

$$
v^{2}=\frac{\mu_{3}^{2}-\mu^{\prime 2}}{2 \lambda_{33}}
$$

is identical to that of the $\mathrm{U}(1)$-symmetric case.

\section{$5.7 \quad \mathrm{Z}_{2} \times \mathrm{Z}_{2}$ symmetric $6 \mathrm{HDM}$ potential}

The mass spectrum of the $Z_{2} \times Z_{2}$ symmetric $6 \mathrm{HDM}$ potential around the minimum point $\left(0,0,0,0, \frac{v}{\sqrt{2}}, \frac{v}{\sqrt{2}}\right)$ with

$$
v^{2}=\frac{\mu_{3}^{2}-\mu^{\prime 2}}{2 \lambda_{33}}
$$

has the following form:

$$
\begin{array}{ll}
\mathbf{G}_{3}=\frac{A_{3 u}^{0}+A_{3 d}^{0}}{\sqrt{2}}: & m^{2}=0 \\
\mathbf{G}_{3}^{ \pm}=\frac{H_{3 u}^{ \pm}+H_{3 d}^{ \pm}}{\sqrt{2}}: & m^{2}=0 \\
\mathbf{h}_{3}=\frac{H_{3 u}^{0}+H_{3 d}^{0}}{\sqrt{2}}: & m^{2}=2 \mu_{3}^{2}-2 \mu^{\prime 2} \\
\mathbf{H}_{3}=\frac{H_{3 u}^{0}-H_{3 d}^{0}}{\sqrt{2}}: & m^{2}=-2 \mu^{\prime 2} \\
\mathbf{A}_{3}=\frac{A_{3 u}^{0}-A_{3 d}^{0}}{\sqrt{2}}: & m^{2}=-2 \mu^{\prime 2} \\
\mathbf{H}_{3}^{ \pm}=\frac{H_{3 u}^{ \pm}-H_{3 d}^{ \pm}}{\sqrt{2}}: & m^{2}=-2 \mu^{\prime 2} \\
\mathbf{h}_{2}=\frac{H_{2 u}^{0}+H_{2 d}^{0}}{\sqrt{2}}: & m^{2}=-\mu_{2}^{2}+\mu^{\prime 2}+\left(\lambda_{23}+\lambda_{23}^{\prime}+\lambda_{2}\right) v^{2}
\end{array}
$$




$$
\begin{array}{ll}
\mathbf{G}_{2}=\frac{A_{2 u}^{0}+A_{2 d}^{0}}{\sqrt{2}}: & m^{2}=-\mu_{2}^{2}+\mu^{\prime 2}+\left(\lambda_{23}+\lambda_{23}^{\prime}-\lambda_{2}\right) v^{2} \\
\mathbf{G}_{2}^{ \pm}=\frac{H_{2 u}^{ \pm}+H_{2 d}^{ \pm}}{\sqrt{2}}: & m^{2}=-\mu_{2}^{2}+\mu^{\prime 2}+\lambda_{23} v^{2} \\
\mathbf{H}_{2}=\frac{H_{2 u}^{0}-H_{2 d}^{0}}{\sqrt{2}}: & m^{2}=-\mu_{2}^{2}-\mu^{\prime 2}+\lambda_{23} v^{2} \\
\mathbf{A}_{2}=\frac{A_{2 u}^{0}-A_{2 d}^{0}}{\sqrt{2}}: & m^{2}=-\mu_{2}^{2}-\mu^{\prime 2}+\lambda_{23} v^{2} \\
\mathbf{H}_{2}^{ \pm}=\frac{H_{2 u}^{ \pm}-H_{2 d}^{ \pm}}{\sqrt{2}}: & m^{2}=-\mu_{2}^{2}-\mu^{\prime 2}+\lambda_{23} v^{2} \\
\mathbf{h}_{1}=\frac{H_{1 u}^{0}+H_{1 d}^{0}}{\sqrt{2}}: & m^{2}=-\mu_{1}^{2}+\mu^{\prime 2}+\left(\lambda_{31}+\lambda_{31}^{\prime}+\lambda_{3}\right) v^{2} \\
\mathbf{G}_{1}=\frac{A_{1 u}^{0}+A_{1 d}^{0}}{\sqrt{2}}: & m^{2}=-\mu_{1}^{2}+\mu^{\prime 2}+\left(\lambda_{31}+\lambda_{31}^{\prime}-\lambda_{3}\right) v^{2} \\
\mathbf{G}_{1}^{ \pm}=\frac{H_{1 u}^{ \pm}+H_{1 d}^{ \pm}}{\sqrt{2}}: & m^{2}=-\mu_{1}^{2}+\mu^{\prime 2}+\lambda_{31} v^{2} \\
\mathbf{H}_{1}=\frac{H_{1 u}^{0}-H_{1 d}^{0}}{\sqrt{2}}: & m^{2}=-\mu_{1}^{2}-\mu^{\prime 2}+\lambda_{31} v^{2} \\
\mathbf{A}_{1}=\frac{A_{1 u}^{0}-A_{1 d}^{0}}{\sqrt{2}}: & m^{2}=-\mu_{1}^{2}-\mu^{\prime 2}+\lambda_{31} v^{2} \\
\mathbf{H}_{1}^{ \pm}=\frac{H_{1 u}^{ \pm}-H_{1 d}^{ \pm}}{\sqrt{2}}: & m^{2}=-\mu_{1}^{2}-\mu^{\prime 2}+\lambda_{31} v^{2}
\end{array}
$$

\section{$5.8 \mathrm{D}_{6}$ symmetric $6 \mathrm{HDM}$ potential}

The mass spectrum of the $D_{6}$ symmetric $6 \mathrm{HDM}$ potential around the minimum point $\left(0,0,0,0, \frac{v}{\sqrt{2}}, \frac{v}{\sqrt{2}}\right)$ with

$$
v^{2}=\frac{\mu_{3}^{2}-\mu^{\prime 2}}{2 \lambda_{33}}
$$

has the following form:

$$
\begin{array}{ll}
\mathbf{G}_{3}=\frac{A_{3 u}^{0}+A_{3 d}^{0}}{\sqrt{2}}: & m^{2}=0 \\
\mathbf{G}_{3}^{ \pm}=\frac{H_{3 u}^{ \pm}+H_{3 d}^{ \pm}}{\sqrt{2}}: & m^{2}=0 \\
\mathbf{h}_{3}=\frac{H_{3 u}^{0}+H_{3 d}^{0}}{\sqrt{2}}: & m^{2}=2 \mu_{3}^{2}-2 \mu^{\prime 2} \\
\mathbf{H}_{3}=\frac{H_{3 u}^{0}-H_{3 d}^{0}}{\sqrt{2}}: & m^{2}=-2 \mu^{\prime 2} \\
\mathbf{A}_{3}=\frac{A_{3 u}^{0}-A_{3 d}^{0}}{\sqrt{2}}: & m^{2}=-2 \mu^{\prime 2} \\
\mathbf{H}_{3}^{ \pm}=\frac{H_{3 u}^{ \pm}-H_{3 d}^{ \pm}}{\sqrt{2}}: & m^{2}=-2 \mu^{\prime 2} \\
\mathbf{G}_{2}^{ \pm}=\frac{H_{2 u}^{ \pm}+H_{2 d}^{ \pm}}{\sqrt{2}}: & m^{2}=-\mu_{12}^{2}+\mu^{\prime 2}+\lambda_{13} v^{2} \\
\mathbf{G}_{1}^{ \pm}=\frac{H_{1 u}^{ \pm}+H_{1 d}^{ \pm}}{\sqrt{2}}: & m^{2}=-\mu_{12}^{2}+\mu^{\prime 2}+\lambda_{13} v^{2} \\
\mathbf{H}_{2}^{ \pm}=\frac{H_{2 u}^{ \pm}-H_{2 d}^{ \pm}}{\sqrt{2}}: & m^{2}=-\mu_{12}^{2}-\mu^{\prime 2}+\lambda_{13} v^{2}
\end{array}
$$




$$
\begin{aligned}
& \mathbf{H}_{1}^{ \pm}=\frac{H_{1 u}^{ \pm}-H_{1 d}^{ \pm}}{\sqrt{2}}: \quad m^{2}=-\mu_{12}^{2}-\mu^{\prime 2}+\lambda_{13} v^{2} \\
& \mathbf{A}_{2}=\frac{A_{2 u}^{0}-A_{2 d}^{0}}{\sqrt{2}}: \quad m^{2}=-\mu_{12}^{2}-\mu^{\prime 2}+\lambda_{13} v^{2} \\
& \mathbf{A}_{1}=\frac{A_{1 u}^{0}-A_{1 d}^{0}}{\sqrt{2}}: \quad m^{2}=-\mu_{12}^{2}-\mu^{\prime 2}+\lambda_{13} v^{2} \\
& \mathbf{H}_{2}=\frac{H_{2 u}^{0}-H_{2 d}^{0}}{\sqrt{2}}: \quad m^{2}=-\mu_{12}^{2}-\mu^{\prime 2}+\lambda_{13} v^{2} \\
& \mathbf{H}_{1}=\frac{H_{1 u}^{0}-H_{1 d}^{0}}{\sqrt{2}}: \quad m^{2}=-\mu_{12}^{2}-\mu^{\prime 2}+\lambda_{13} v^{2} \\
& \mathbf{G}_{2}^{\prime}=\frac{A_{2 u}^{0}+A_{2 d}^{0}+A_{1 u}^{0}+A_{1 d}^{0}}{2}: \quad m^{2}=-\mu_{12}^{2}+\mu^{\prime 2}+\left(\lambda_{13}+\lambda_{13}^{\prime}-\lambda_{2}\right) v^{2} \\
& \mathbf{G}_{1}^{\prime}=\frac{-A_{2 u}^{0}-A_{2 d}^{0}+A_{1 u}^{0}+A_{1 d}^{0}}{\sqrt{2}}: \quad m^{2}=-\mu_{12}^{2}+\mu^{\prime 2}+\left(\lambda_{13}+\lambda_{13}^{\prime}+\lambda_{2}\right) v^{2} \\
& \mathbf{h}_{2}^{\prime}=\frac{H_{2 u}^{0}+H_{2 d}^{0}+H_{1 u}^{0}+H_{1 d}^{0}}{2}: \quad m^{2}=-\mu_{12}^{2}+\mu^{\prime 2}+\left(\lambda_{13}+\lambda_{13}^{\prime}-\lambda_{2}\right) v^{2} \\
& \mathbf{h}_{1}^{\prime}=\frac{-H_{2 u}^{0}-H_{2 d}^{0}+H_{1 u}^{0}+H_{1 d}^{0}}{2}: \quad m^{2}=-\mu_{12}^{2}+\mu^{\prime 2}+\left(\lambda_{13}+\lambda_{13}^{\prime}+\lambda_{2}\right) v^{2}
\end{aligned}
$$

\section{$5.9 \quad D_{8}$ symmetric 6 HDM potential}

The mass spectrum of the $D_{8}$ symmetric $6 \mathrm{HDM}$ potential around the minimum point $\left(0,0,0,0, \frac{v}{\sqrt{2}}, \frac{v}{\sqrt{2}}\right)$ with

$$
v^{2}=\frac{\mu_{3}^{2}-\mu^{\prime 2}}{2 \lambda_{33}}
$$

is the same as the $D_{6}$-symmetric case with the slight difference in the definition of $a, b$ and $Y$ (replace $\lambda_{2}$ by $\left.\lambda_{1}\right)$.

\section{$5.10 \quad \mathrm{~A}_{4}$ symmetric $6 \mathrm{HDM}$ potential}

The mass spectrum of the $A_{4}$ symmetric $6 \mathrm{HDM}$ potential around the minimum point $\left(0,0,0,0, \frac{v}{\sqrt{2}}, \frac{v}{\sqrt{2}}\right)$ with

$$
v^{2}=\frac{\mu_{3}^{2}-\mu^{\prime 2}}{2 \lambda_{11}}
$$

has the following form:

$$
\begin{array}{ll}
\mathbf{G}_{3}=\frac{A_{3 u}^{0}+A_{3 d}^{0}}{\sqrt{2}}: & m^{2}=0 \\
\mathbf{G}_{3}^{ \pm}=\frac{H_{3 u}^{ \pm}+H_{3 d}^{ \pm}}{\sqrt{2}}: & m^{2}=0 \\
\mathbf{h}_{3}=\frac{H_{3 u}^{0}+H_{3 d}^{0}}{\sqrt{2}}: & m^{2}=2 \mu^{2}-2 \mu^{\prime 2} \\
\mathbf{A}_{3}=\frac{A_{3 u}^{0}-A_{3 d}^{0}}{\sqrt{2}}: & m^{2}=-2 \mu^{\prime 2} \\
\mathbf{H}_{3}^{ \pm}=\frac{H_{3 u}^{ \pm}-H_{3 d}^{ \pm}}{\sqrt{2}}: & m^{2}=-2 \mu^{\prime 2} \\
\mathbf{H}_{3}=\frac{H_{3 u}^{0}-H_{3 d}^{0}}{\sqrt{2}}: & m^{2}=-2 \mu^{\prime 2}
\end{array}
$$




$$
\begin{aligned}
& \mathbf{G}_{2}^{ \pm}=\frac{H_{2 u}^{ \pm}+H_{2 d}^{ \pm}}{\sqrt{2}}: \quad m^{2}=-\mu^{2}+\mu^{\prime 2}+\left(\lambda_{12}+2 \lambda_{11}\right) v^{2} \\
& \mathbf{G}_{1}^{ \pm}=\frac{H_{1 u}^{ \pm}+H_{1 d}^{ \pm}}{\sqrt{2}}: \quad m^{2}=-\mu^{2}+\mu^{\prime 2}+\left(\lambda_{12}+2 \lambda_{11}\right) v^{2} \\
& \mathbf{H}_{2}^{ \pm}=\frac{H_{2 u}^{ \pm}-H_{2 d}^{ \pm}}{\sqrt{2}}: \quad m^{2}=-\mu^{2}-\mu^{\prime 2}+\left(\lambda_{12}+2 \lambda_{11}\right) v^{2} \\
& \mathbf{H}_{1}^{ \pm}=\frac{H_{1 u}^{ \pm}-H_{1 d}^{ \pm}}{\sqrt{2}}: \quad m^{2}=-\mu^{2}-\mu^{\prime 2}+\left(\lambda_{12}+2 \lambda_{11}\right) v^{2} \\
& \mathbf{H}_{2}=\frac{H_{2 u}^{0}-H_{2 d}^{0}}{\sqrt{2}}: \quad m^{2}=\mu^{2}-\mu^{\prime 2}+\frac{1}{2}\left(\lambda_{12}+2 \lambda_{11}\right) v^{2} \\
& \mathbf{H}_{1}=\frac{H_{1 u}^{0}-H_{1 d}^{0}}{\sqrt{2}}: \quad m^{2}=\mu^{2}-\mu^{\prime 2}+\frac{1}{2}\left(\lambda_{12}+2 \lambda_{11}\right) v^{2} \\
& \mathbf{A}_{2}=\frac{A_{2 u}^{0}-A_{2 d}^{0}}{\sqrt{2}}: \quad m^{2}=\mu^{2}-\mu^{\prime 2}+\frac{1}{2}\left(\lambda_{12}+2 \lambda_{11}\right) v^{2} \\
& \mathbf{A}_{1}=\frac{A_{1 u}^{0}-A_{1 d}^{0}}{\sqrt{2}}: \quad m^{2}=\mu^{2}-\mu^{\prime 2}+\frac{1}{2}\left(\lambda_{12}+2 \lambda_{11}\right) v^{2} \\
& \mathbf{h}_{2}^{\prime}=\frac{a H_{2 u}^{0}+a H_{2 d}^{0}+A_{2 u}^{0}+A_{2 d}^{0}}{\sqrt{2+2 a^{2}}}: \quad m^{2}=-\mu^{2}+\mu^{\prime 2}+\left(\lambda_{12}+2 \lambda_{11}+\lambda_{12}^{\prime}+\operatorname{Re} \lambda_{1}-2 \sqrt{2} \operatorname{Im} \lambda_{1}\right) v^{2} \\
& \mathbf{h}_{1}^{\prime}=\frac{a H_{1 u}^{0}+a H_{1 d}^{0}+A_{1 u}^{0}+A_{1 d}^{0}}{\sqrt{2+2 a^{2}}}: \quad m^{2}=-\mu^{2}+\mu^{\prime 2}+\left(\lambda_{12}+2 \lambda_{11}+\lambda_{12}^{\prime}+\operatorname{Re} \lambda_{1}-2 \sqrt{2} \operatorname{Im} \lambda_{1}\right) v^{2} \\
& \mathbf{G}_{2}^{\prime}=\frac{b H_{2 u}^{0}+b H_{2 d}^{0}+A_{2 u}^{0}+A_{2 d}^{0}}{\sqrt{2+2 b^{2}}}: \quad m^{2}=-\mu^{2}+\mu^{\prime 2}+\left(\lambda_{12}+2 \lambda_{11}+\lambda_{12}^{\prime}+\operatorname{Re} \lambda_{1}+2 \sqrt{2} \operatorname{Im} \lambda_{1}\right) v^{2} \\
& \mathbf{G}_{1}^{\prime}=\frac{b H_{1 u}^{0}+b H_{1 d}^{0}+A_{1 u}^{0}+A_{1 d}^{0}}{\sqrt{2+2 b^{2}}}: \quad m^{2}=-\mu^{2}+\mu^{\prime 2}+\left(\lambda_{12}+2 \lambda_{11}+\lambda_{12}^{\prime}+\operatorname{Re} \lambda_{1}+2 \sqrt{2} \operatorname{Im} \lambda_{1}\right) v^{2} \\
& \text { where } a=(1-\sqrt{2}) \quad \text { and } \quad b=(1+\sqrt{2})
\end{aligned}
$$

\section{$5.11 \mathrm{~S}_{4}$ symmetric $6 \mathrm{HDM}$ potential}

The mass spectrum of the $S_{4}$ symmetric $6 \mathrm{HDM}$ potential around the minimum point $\left(0,0,0,0, \frac{v}{\sqrt{2}}, \frac{v}{\sqrt{2}}\right)$ with

$$
v^{2}=\frac{\mu_{3}^{2}-\mu^{\prime 2}}{2 \lambda_{11}}
$$

has the following form:

$$
\begin{array}{ll}
\mathbf{G}_{3}=\frac{A_{3 u}^{0}+A_{3 d}^{0}}{\sqrt{2}}: & m^{2}=0 \\
\mathbf{G}_{3}^{ \pm}=\frac{H_{3 u}^{ \pm}+H_{3 d}^{ \pm}}{\sqrt{2}}: & m^{2}=0 \\
\mathbf{h}_{3}=\frac{H_{3 u}^{0}+H_{3 d}^{0}}{\sqrt{2}}: & m^{2}=2 \mu^{2}-2 \mu^{\prime 2} \\
\mathbf{A}_{3}=\frac{A_{3 u}^{0}-A_{3 d}^{0}}{\sqrt{2}}: & m^{2}=-2 \mu^{\prime 2} \\
\mathbf{H}_{3}^{ \pm}=\frac{H_{3 u}^{ \pm}-H_{3 d}^{ \pm}}{\sqrt{2}}: & m^{2}=-2 \mu^{\prime 2} \\
\mathbf{H}_{3}=\frac{H_{3 u}^{0}-H_{3 d}^{0}}{\sqrt{2}}: & m^{2}=-2 \mu^{\prime 2} \\
\mathbf{G}_{2}^{ \pm}=\frac{H_{2 u}^{ \pm}+H_{2 d}^{ \pm}}{\sqrt{2}}: & m^{2}=-\mu^{2}+\mu^{\prime 2}+\left(\lambda_{12}+2 \lambda_{11}\right) v^{2}
\end{array}
$$




$$
\begin{array}{ll}
\mathbf{G}_{1}^{ \pm}=\frac{H_{1 u}^{ \pm}+H_{1 d}^{ \pm}}{\sqrt{2}}: & m^{2}=-\mu^{2}+\mu^{\prime 2}+\left(\lambda_{12}+2 \lambda_{11}\right) v^{2} \\
\mathbf{H}_{2}^{ \pm}=\frac{H_{2 u}^{ \pm}-H_{2 d}^{ \pm}}{\sqrt{2}}: & m^{2}=-\mu^{2}-\mu^{\prime 2}+\left(\lambda_{12}+2 \lambda_{11}\right) v^{2} \\
\mathbf{H}_{1}^{ \pm}=\frac{H_{1 u}^{ \pm}-H_{1 d}^{ \pm}}{\sqrt{2}}: & m^{2}=-\mu^{2}-\mu^{\prime 2}+\left(\lambda_{12}+2 \lambda_{11}\right) v^{2} \\
\mathbf{h}_{2}=\frac{H_{2 u}^{0}+H_{2 d}^{0}}{\sqrt{2}}: & m^{2}=-\mu^{2}+\mu^{\prime 2}+\left(\lambda_{12}+2 \lambda_{11}+\lambda_{12}^{\prime}+\lambda_{1}\right) v^{2} \\
\mathbf{h}_{1}=\frac{H_{1 u}^{0}+H_{1 d}^{0}}{\sqrt{2}}: & m^{2}=-\mu^{2}+\mu^{\prime 2}+\left(\lambda_{12}+2 \lambda_{11}+\lambda_{12}^{\prime}+\lambda_{1}\right) v^{2} \\
\mathbf{A}_{2}=\frac{A_{2 u}^{0}-A_{2 d}^{0}}{\sqrt{2}}: & m^{2}=-\mu^{2}-\mu^{\prime 2}+\left(\lambda_{12}+2 \lambda_{11}\right) v^{2} \\
\mathbf{A}_{1}=\frac{A_{1 u}^{0}-A_{1 d}^{0}}{\sqrt{2}}: & m^{2}=-\mu^{2}-\mu^{\prime 2}+\left(\lambda_{12}+2 \lambda_{11}\right) v^{2} \\
\mathbf{H}_{2}=\frac{H_{2 u}^{0}-H_{2 d}^{0}}{\sqrt{2}}: & m^{2}=-\mu^{2}-\mu^{\prime 2}+\left(\lambda_{12}+2 \lambda_{11}\right) v^{2} \\
\mathbf{H}_{1}=\frac{H_{1 u}^{0}-H_{1 d}^{0}}{\sqrt{2}}: & m^{2}=-\mu^{2}-\mu^{\prime 2}+\left(\lambda_{12}+2 \lambda_{11}\right) v^{2} \\
\mathbf{G}_{2}=\frac{A_{2 u}^{0}+A_{2 d}^{0}}{\sqrt{2}}: & m^{2}=-\mu^{2}+\mu^{\prime 2}+\left(\lambda_{12}+2 \lambda_{11}+\lambda_{12}^{\prime}-\lambda_{1}\right) v^{2} \\
\mathbf{G}_{1}=\frac{A_{1 u}^{0}+A_{1 d}^{0}}{\sqrt{2}}: & m^{2}=-\mu^{2}+\mu^{\prime 2}+\left(\lambda_{12}+2 \lambda_{11}+\lambda_{12}^{\prime}-\lambda_{1}\right) v^{2}
\end{array}
$$

\section{$5.12 \Delta(54) / \mathrm{Z}_{3}$ symmetric $6 \mathrm{HDM}$ potential}

The mass spectrum of the $\Delta(54) / Z_{3}$ symmetric $6 \mathrm{HDM}$ potential around the minimum point $\left(0,0,0,0, \frac{v}{\sqrt{2}}, \frac{v}{\sqrt{2}}\right)$ with

$$
v^{2}=\frac{\mu^{2}-\mu^{\prime 2}}{2\left(\lambda_{11}+\lambda_{12}\right)}
$$

has the following form:

$$
\begin{array}{ll}
\mathbf{G}_{3}=\frac{A_{3 u}^{0}+A_{3 d}^{0}}{\sqrt{2}}: & m^{2}=0 \\
\mathbf{G}_{3}^{ \pm}=\frac{H_{3 u}^{ \pm}+H_{3 d}^{ \pm}}{\sqrt{2}}: & m^{2}=0 \\
\mathbf{h}_{3}=\frac{H_{3 u}^{0}+H_{3 d}^{0}}{\sqrt{2}}: & m^{2}=2 \mu^{2}-2 \mu^{\prime 2} \\
\mathbf{H}_{3}=\frac{H_{3 u}^{0}-H_{3 d}^{0}}{\sqrt{2}}: & m^{2}=-2 \mu^{\prime 2} \\
\mathbf{A}_{3}=\frac{A_{3 u}^{0}-A_{3 d}^{0}}{\sqrt{2}}: & m^{2}=-2 \mu^{\prime 2} \\
\mathbf{H}_{3}^{ \pm}=\frac{H_{3 u}^{ \pm}-H_{3 d}^{ \pm}}{\sqrt{2}}: & m^{2}=-2 \mu^{\prime 2} \\
\mathbf{H}_{2}^{ \pm}=\frac{H_{2 u}^{ \pm}-H_{2 d}^{ \pm}}{\sqrt{2}}: & m^{2}=-\mu^{2}-\mu^{\prime 2}+\left(2 \lambda_{11}-\lambda_{12}\right) v^{2} \\
\mathbf{H}_{1}^{ \pm}=\frac{H_{1 u}^{ \pm}-H_{1 d}^{ \pm}}{\sqrt{2}}: & m^{2}=-\mu^{2}-\mu^{\prime 2}+\left(2 \lambda_{11}-\lambda_{12}\right) v^{2} \\
\mathbf{G}_{2}^{ \pm}=\frac{H_{2 u}^{ \pm}+H_{2 d}^{ \pm}}{\sqrt{2}}: & m^{2}=-\mu^{2}+\mu^{\prime 2}+\left(2 \lambda_{11}-\lambda_{12}\right) v^{2}
\end{array}
$$




$$
\begin{aligned}
& \mathbf{G}_{1}^{ \pm}=\frac{H_{1 u}^{ \pm}+H_{1 d}^{ \pm}}{\sqrt{2}}: \quad m^{2}=-\mu^{2}+\mu^{\prime 2}+\left(2 \lambda_{11}-\lambda_{12}\right) v^{2} \\
& \mathbf{H}_{2}=\frac{H_{2 u}^{0}-H_{2 d}^{0}}{\sqrt{2}}: \quad m^{2}=-\mu^{2}-\mu^{\prime 2}+\left(2 \lambda_{11}-\lambda_{12}\right) v^{2} \\
& \mathbf{A}_{2}=\frac{A_{2 u}^{0}-A_{2 d}^{0}}{\sqrt{2}}: \quad m^{2}=-\mu^{2}-\mu^{\prime 2}+\left(2 \lambda_{11}-\lambda_{12}\right) v^{2} \\
& \mathbf{H}_{1}=\frac{H_{1 u}^{0}-H_{1 d}^{0}}{\sqrt{2}}: \quad m^{2}=-\mu^{2}-\mu^{\prime 2}+\left(2 \lambda_{11}-\lambda_{12}\right) v^{2} \\
& \mathbf{A}_{1}=\frac{A_{1 u}^{0}-A_{1 d}^{0}}{\sqrt{2}}: \quad m^{2}=-\mu^{2}-\mu^{\prime 2}+\left(2 \lambda_{11}-\lambda_{12}\right) v^{2} \\
& \mathbf{h}_{2}^{\prime}=\frac{W H_{2 u}^{0}+W H_{2 d}^{0}-X H_{1 u}^{0}-X H_{1 d}^{0}+A_{2 u}^{0}+A_{2 d}^{0}}{\sqrt{2+X^{2}+2 W^{2}}}: \\
& m^{2}=-\mu^{2}+\mu^{\prime 2}+\left(2 \lambda_{11}-\lambda_{12}+\lambda_{12}^{\prime}-\frac{1}{2} \sqrt{\operatorname{Re}^{2} \lambda_{1}+\operatorname{Im}^{2} \lambda_{1}}\right) v^{2} \\
& \mathbf{h}_{1}^{\prime}=\frac{W H_{2 u}^{0}+W H_{2 d}^{0}+X H_{1 u}^{0}+X H_{1 d}^{0}+A_{2 u}^{0}+A_{2 d}^{0}}{\sqrt{2+X^{2}+2 W^{2}}}: \\
& m^{2}=-\mu^{2}+\mu^{\prime 2}+\left(2 \lambda_{11}-\lambda_{12}+\lambda_{12}^{\prime}+\frac{1}{2} \sqrt{\operatorname{Re}^{2} \lambda_{1}+\operatorname{Im}^{2} \lambda_{1}}\right) v^{2} \\
& \mathbf{G}_{2}^{\prime}=\frac{-W^{\prime} H_{1 u}^{0}-W^{\prime} H_{1 d}^{0}-(Y+Z) A_{2 u}^{0}+(Y-Z) A_{2 d}^{0}+A_{1 u}^{0}+A_{1 d}^{0}}{\sqrt{2+(Y+Z)^{2}+(Y-Z)^{2}+2 W^{\prime 2}}}: \\
& m^{2}=-\mu^{2}+\mu^{\prime 2}+\left(2 \lambda_{11}-\lambda_{12}+\lambda_{12}^{\prime}-\frac{1}{4} \sqrt{2 \operatorname{Re}^{2} \lambda_{1}+4 \operatorname{Im}^{2} \lambda_{1}}\right) v^{2} \\
& \mathbf{G}_{1}^{\prime}=\frac{W^{\prime} H_{1 u}^{0}+W^{\prime} H_{1 d}^{0}-(Y-Z) A_{2 u}^{0}+(Y+Z) A_{2 d}^{0}+A_{1 u}^{0}+A_{1 d}^{0}}{\sqrt{2+(Y+Z)^{2}+(Y-Z)^{2}+2 W^{\prime 2}}}: \\
& m^{2}=-\mu^{2}+\mu^{\prime 2}+\left(2 \lambda_{11}-\lambda_{12}+\lambda_{12}^{\prime}+\frac{1}{4} \sqrt{2 \operatorname{Re}^{2} \lambda_{1}+4 \operatorname{Im}^{2} \lambda_{1}}\right) v^{2} \\
& \text { where } \quad W=\frac{\operatorname{Re} \lambda_{1}}{\operatorname{Im} \lambda_{1}} \quad \text { and } \quad W^{\prime}=\frac{\operatorname{Im} \lambda_{1}}{\operatorname{Re} \lambda_{1}} \\
& X=\frac{\sqrt{\operatorname{Re}^{2} \lambda_{1}+\operatorname{Im}^{2} \lambda_{1}}}{\operatorname{Im} \lambda_{1}} \\
& Y=\frac{\operatorname{Re} \lambda_{1}\left(2 \mu^{\prime 2} / v^{2}+\lambda_{12}^{\prime}\right)}{-2\left(2 \mu^{\prime 2} / v^{2}+\lambda_{12}^{\prime}\right)^{2}+\operatorname{Re}^{2} \lambda_{1}+2 \operatorname{Im}^{2} \lambda_{1}} \\
& Z=\frac{\left(2 \mu^{\prime 2} / v^{2}+\lambda_{12}^{\prime}\right)^{2} \sqrt{2 \operatorname{Re}^{2} \lambda_{1}+4 \operatorname{Im}^{2} \lambda_{1}}}{\left[-2\left(2 \mu^{\prime 2} / v^{2}+\lambda_{12}^{\prime}\right)^{2}+\operatorname{Re}^{2} \lambda_{1}+2 \operatorname{Im}^{2} \lambda_{1}\right] \operatorname{Re} \lambda_{1}}
\end{aligned}
$$

\section{$5.13 \Sigma(36)$ symmetric 6 HDM potential}

The mass spectrum of the $\Sigma(36)$ symmetric $6 \mathrm{HDM}$ potential around the minimum point $\left(0,0,0,0, \frac{v}{\sqrt{2}}, \frac{v}{\sqrt{2}}\right)$ with

$$
v^{2}=\frac{\mu^{2}-\mu^{\prime 2}}{2 \lambda_{11}}
$$

has the following form:

$$
\begin{aligned}
\mathbf{G}_{3}=\frac{A_{3 u}^{0}+A_{3 d}^{0}}{\sqrt{2}}: & m^{2}=0 \\
\mathbf{G}_{3}^{ \pm}=\frac{H_{3 u}^{ \pm}+H_{3 d}^{ \pm}}{\sqrt{2}}: & m^{2}=0
\end{aligned}
$$




$$
\begin{array}{ll}
\mathbf{G}_{2}^{ \pm}=\frac{H_{2 u}^{ \pm}+H_{2 d}^{ \pm}}{\sqrt{2}}: & m^{2}=-\mu^{2}+\mu^{\prime 2}+2 \lambda_{11} v^{2} \\
\mathbf{G}_{1}^{ \pm}=\frac{H_{1 u}^{ \pm}+H_{1 d}^{ \pm}}{\sqrt{2}}: & m^{2}=-\mu^{2}+\mu^{\prime 2}+2 \lambda_{11} v^{2} \\
\mathbf{h}_{3}=\frac{H_{3 u}^{0}+H_{3 d}^{0}}{\sqrt{2}}: & m^{2}=2 \mu^{2}-2 \mu^{\prime 2} \\
\mathbf{H}_{3}=\frac{H_{3 u}^{0}-H_{3 d}^{0}}{\sqrt{2}}: & m^{2}=-2 \mu^{\prime 2} \\
\mathbf{A}_{3}=\frac{A_{3 u}^{0}-A_{3 d}^{0}}{\sqrt{2}}: & m^{2}=-2 \mu^{\prime 2} \\
\mathbf{H}_{2}=\frac{H_{2 u}^{0}-H_{2 d}^{0}}{\sqrt{2}}: & m^{2}=-2 \mu^{\prime 2} \\
\mathbf{A}_{2}=\frac{A_{2 u}^{0}-A_{2 d}^{0}}{\sqrt{2}}: & m^{2}=-2 \mu^{\prime 2} \\
\mathbf{H}_{1}=\frac{H_{1 u}^{0}-H_{1 d}^{0}}{\sqrt{2}}: & m^{2}=-2 \mu^{\prime 2} \\
\mathbf{A}_{1}=\frac{A_{1 u}^{0}-A_{1 d}^{0}}{\sqrt{2}}: & m^{2}=-2 \mu^{\prime 2} \\
\mathbf{H}_{3}^{ \pm}=\frac{H_{3 u}^{ \pm}-H_{3 d}^{ \pm}}{\sqrt{2}}: & m^{2}=-2 \mu^{\prime 2} \\
\mathbf{H}_{2}^{ \pm}=\frac{H_{2 u}^{ \pm}-H_{2 d}^{ \pm}}{\sqrt{2}}: & m^{2}=-2 \mu^{\prime 2} \\
\mathbf{H}_{1}^{ \pm}=\frac{H_{1 u}^{ \pm}-H_{1 d}^{ \pm}}{\sqrt{2}}: & m^{2}=-2 \mu^{\prime 2} \\
\mathbf{h}_{2}=\frac{H_{2 u}^{0}+H_{2 d}^{0}}{\sqrt{2}}: & m^{2}=2 \lambda_{12}^{\prime} v^{2} \\
\mathbf{G}_{2}=\frac{A_{2 u}^{0}+A_{2 d}^{0}}{\sqrt{2}}: & m^{2}=2 \lambda_{12}^{\prime} v^{2} \\
\mathbf{h}_{1}=\frac{H_{1 u}^{0}+H_{1 d}^{0}}{\sqrt{2}}: & m^{2}=2 \lambda_{12}^{\prime} v^{2} \\
\mathbf{G}_{1}=\frac{A_{1 u}^{0}+A_{1 d}^{0}}{\sqrt{2}}: & m^{2}=2 \lambda_{12}^{\prime} v^{2} \\
&
\end{array}
$$

\section{Conclusion}

In this paper we have considered 3HDMs which are the next simplest class of models, following the well studied 2HDMs. We have argued that 3HDMs are ready for serious investigation since their possible symmetries have been largely identified. Furthermore, they may shed light on the flavour problem, in the sense that their symmetries may be identified as family symmetries which also describe the three families of quarks and leptons.

We have catalogued and studied 3HDMs in terms of all possible allowed symmetries (continuous and discrete Abelian and discrete non-Abelian). We have analysed the potential in each case, and derived the conditions under which the vacuum alignments $(0,0, v)$, $(0, v, v)$ and $(v, v, v)$ are minima of the potential. For the alignment $(0,0, v)$, relevant for DM models, we have calculated the corresponding physical Higgs boson mass spectrum. 
Motivated by SUSY, we have extended the analysis to the case of three up-type Higgs doublets and three down-type Higgs doublets (six doublets in total), for the case of $\tan \beta=$ 1. Many of the results are also applicable to flavon models where the three Higgs doublets are replaced by three electroweak singlets.

In conclusion, following the discovery of a Higgs boson by the LHC, it is clear that Nature admits at least one Higgs doublet for the purpose of breaking electroweak symmetry. However it is not yet clear if there are two or more Higgs doublets which are relevant in Nature. We have systematically studied the case of 3HDMs, whose symmetries may shed light on the flavour problem. If SUSY is relevant, then we have shown how the analysis may be straightforwardly extended to $6 \mathrm{HDMs}$.

\section{Acknowledgments}

VK acknowledges numerous useful discussions with Andrew G. Akeroyd and Alexander Merle. VK is supported by The Leverhulme Trust (London, U.K.) via a Visiting Fellowship. SM is financed in part through the NExT Institute and from the STFC Consolidated ST/J000396/1. SFK also acknowledges partial support from the STFC Consolidated ST/J000396/1 and EU ITN grants UNILHC 237920 and INVISIBLES 289442.

\section{A Finding all realisable symmetries}

\section{A.1 Abelian symmetries}

As mentioned in section 2 , the group of physically distinct unitary reparametrisation transformations respected by the kinetic terms $G_{0}$ in $3 \mathrm{HDMs}$ is

$$
G_{0}=\mathrm{PSU}(3) \simeq \mathrm{SU}(3) / \mathbb{Z}_{3}
$$

To find all realisable Abelian subgroups of $G_{0}$, one first needs to construct the maximal Abelian subgroups of PSU(3) and then explore the realisable subgroups of the maximal Abelian subgroup.

A maximal Abelian subgroup of $G_{0}$ is an Abelian group that is not contained in any larger Abelian subgroup of $G_{0}$. In principal, $G_{0}$ can have several maximal Abelian subgroups and any subgroup of $G_{0}$ is either a subgroup of a maximal Abelian subgroup or is itself a maximal Abelian subgroup.

Within $\mathrm{SU}(N)$, it is known that all maximal Abelian subgroups are maximal tori [12]:

$$
[\mathrm{U}(1)]^{N-1}=\mathrm{U}(1) \times \mathrm{U}(1) \times \cdots \times \mathrm{U}(1) .
$$

All such maximal tori are conjugate to each other. ${ }^{10}$ Therefore, without loss of generality, we could pick one specific maximal torus and study its subgroup. It is convenient to pick the maximal torus represented by phase rotations of individual doublets:

$$
\operatorname{diag}\left(e^{i \alpha_{1}}, e^{i \alpha_{2}}, \ldots, e^{i \alpha_{N-1}}, e^{-i \sum \alpha_{i}}\right) .
$$

\footnotetext{
${ }^{10}$ If $T_{1}$ and $T_{2}$ are two maximal tori, there exists $g \in \mathrm{SU}(N)$ such that $g^{-1} T_{1} g=T_{2}$.
} 
This transformation can be written as the vector of phases,

$$
\left(\alpha_{1}, \alpha_{2}, \ldots, \alpha_{N-1},-\sum \alpha_{i}\right)
$$

Therefore, the maximal torus inside $\mathrm{SU}(N)$ has the following form:

$$
T_{0}=\mathrm{U}(1)_{1} \times \mathrm{U}(1)_{2} \times \cdots \times \mathrm{U}(1)_{N-1}
$$

where

$$
\begin{aligned}
\mathrm{U}(1)_{1}= & \alpha_{1}(-1,1,0,0, \ldots, 0) \\
\mathrm{U}(1)_{2}= & \alpha_{2}(-2,1,1,0, \ldots, 0) \\
\mathrm{U}(1)_{3}= & \alpha_{3}(-3,1,1,1, \ldots, 0) \\
\vdots & \vdots \\
\mathrm{U}(1)_{N-1}= & \alpha_{N-1}(-N+1,1,1,1, \ldots, 1)
\end{aligned}
$$

with all $\alpha_{i} \in[0,2 \pi)$. However, the center of $\mathrm{SU}(N)$, which is generated by $\alpha_{N-1}=2 \pi / N$ and results in trivial transformations, is contained in $\mathrm{U}(1)_{N-1}$. Therefore we introduce

$$
\overline{\mathrm{U}(1)}_{N-1}=\mathrm{U}(1)_{N-1} / Z(\mathrm{SU}(N))=\alpha_{N-1}\left(-\frac{N-1}{N}, \frac{1}{N}, \ldots, \frac{1}{N}\right)
$$

where $\alpha_{N-1} \in[0,2 \pi)$.

Thus, the maximal torus in $\operatorname{PSU}(N)$ appears as follows:

$$
T=\mathrm{U}(1)_{1} \times \mathrm{U}(1)_{2} \times \cdots \times \overline{\mathrm{U}(1)}_{N-1}
$$

Having constructed the maximal torus within $\operatorname{PSU}(N)$, one needs to exhaust the list of realisable Abelian subgroups of $T$, which is done by adding bilinear terms to the most general $T$-symmetric potential:

$$
V_{0}=\sum_{i}^{N}\left[-\left|\mu_{i}^{2}\right|\left(\phi_{i}^{\dagger} \phi_{i}\right)+\lambda_{i i}\left(\phi_{i}^{\dagger} \phi_{i}\right)^{2}\right]+\sum_{i j}^{N}\left[\lambda_{i j}\left(\phi_{i}^{\dagger} \phi_{i}\right)\left(\phi_{j}^{\dagger} \phi_{j}\right)+\lambda_{i j}^{\prime}\left(\phi_{i}^{\dagger} \phi_{j}\right)\left(\phi_{j}^{\dagger} \phi_{i}\right)\right] .
$$

and checking the symmetries of the resulting potential.

Each bilinear $\phi_{a}^{\dagger} \phi_{b}(a \neq b)$, gets a phase change under $T$ (A.6):

$$
\left(\phi_{a}^{\dagger} \phi_{b}\right) \rightarrow \exp \left[i\left(m_{1} \alpha_{1}+m_{2} \alpha_{2}+\cdots+m_{N-1} \alpha_{N-1}\right)\right]\left(\phi_{a}^{\dagger} \phi_{b}\right)
$$

with integer coefficients $m_{1}, m_{2}, \ldots, m_{N-1}$. This linear dependence on the angles $\alpha_{j}$ could be written as

$$
\sum_{j=1}^{N-1} m_{j} \alpha_{j}
$$

The phase transformation properties of a given monomial are fully described by its vector $m_{j}$, an the phase transformation properties of the potential $V$, which is a collection of $k$ monomials, is characterized by $k$ vectors $m_{1, j}, m_{2, j}, \ldots, m_{k, j}$. 
For a monomial to be invariant under a given transformation defined by phases $\left\{\alpha_{j}\right\}$, one requires that

$$
\sum_{j=1}^{N} m_{j} \alpha_{j}=2 \pi n
$$

with some integer $n$. For an entire potential of $k$ terms to be invariant under a given phase transformation, one requires:

$$
\sum_{j=1}^{N} m_{i, j} \alpha_{j}=2 \pi n_{i}, \quad \text { for all } 1 \leq i \leq k
$$

with integer $n_{i}$ s. In order to solve this set of equations, one could construct the matrix of coefficients $m_{i, j}$ with all integer entries, and diagonalize it, reducing the set of equations to:

$$
m_{i, i}^{\prime} \alpha_{i}^{\prime}=2 \pi n_{i}^{\prime}, \quad \alpha_{i}^{\prime} \in[0,2 \pi), \quad n_{i}^{\prime} \in \mathbb{Z}
$$

with non-negative integers $m_{i, i}^{\prime}$.

- If $m_{i, i}=0$, this equation has a solution for any $\alpha_{i}$; the $i$-th equation contributes a factor $\mathrm{U}(1)$ to the symmetry group of the potential.

- If $m_{i, i}=1$, this equation has no non-trivial solution; the $i$-th equation does not contribute to the symmetry group of the potential.

- If $m_{i, i}=d_{i}>1$, this equation has $d_{i}$ solutions $\alpha_{i}=2 \pi / d_{i}$; the $i$-th equation contributes the factor $\mathbb{Z}_{d_{i}}$ to the symmetry group of the potential.

The full symmetry group of the potential is then constructed from the direct product of the above factors.

Following this strategy for $3 \mathrm{HDMs}$, we

- construct the maximal torus $T \subset \mathrm{PSU}(3)$ :

$$
T=\mathrm{U}(1)_{1} \times \mathrm{U}(1)_{2}, \quad \mathrm{U}(1)_{1}=\alpha(-1,1,0), \quad \mathrm{U}(1)_{2}=\beta\left(-\frac{2}{3}, \frac{1}{3}, \frac{1}{3}\right)
$$

where $\alpha, \beta \in[0,2 \pi)$,

- write down the most general $T$-symmetrci potential:

$$
\begin{aligned}
V_{0}= & -\mu_{1}^{2}\left(\phi_{1}^{\dagger} \phi_{1}\right)-\mu_{2}^{2}\left(\phi_{2}^{\dagger} \phi_{2}\right)-\mu_{3}^{2}\left(\phi_{3}^{\dagger} \phi_{3}\right) \\
& +\lambda_{11}\left(\phi_{1}^{\dagger} \phi_{1}\right)^{2}+\lambda_{22}\left(\phi_{2}^{\dagger} \phi_{2}\right)^{2}+\lambda_{33}\left(\phi_{3}^{\dagger} \phi_{3}\right)^{2} \\
& +\lambda_{12}\left(\phi_{1}^{\dagger} \phi_{1}\right)\left(\phi_{2}^{\dagger} \phi_{2}\right)+\lambda_{23}\left(\phi_{2}^{\dagger} \phi_{2}\right)\left(\phi_{3}^{\dagger} \phi_{3}\right)+\lambda_{31}\left(\phi_{3}^{\dagger} \phi_{3}\right)\left(\phi_{1}^{\dagger} \phi_{1}\right) \\
& +\lambda_{12}^{\prime}\left(\phi_{1}^{\dagger} \phi_{2}\right)\left(\phi_{2}^{\dagger} \phi_{1}\right)+\lambda_{23}^{\prime}\left(\phi_{2}^{\dagger} \phi_{3}\right)\left(\phi_{3}^{\dagger} \phi_{2}\right)+\lambda_{31}^{\prime}\left(\phi_{3}^{\dagger} \phi_{1}\right)\left(\phi_{1}^{\dagger} \phi_{3}\right),
\end{aligned}
$$

- add to the $T$-symmetric potential any combination (quadratic and quartic) of the following doublets transforming non-trivially under $T$,

$$
\left(\phi_{1}^{\dagger} \phi_{2}\right), \quad\left(\phi_{2}^{\dagger} \phi_{3}\right), \quad\left(\phi_{3}^{\dagger} \phi_{1}\right)
$$

and their conjugates, 
- construct the matrix of coefficients $m_{i j}$ for all the potentials and diagonalize it,

and arrive at the full list of subgroups of the maximal torus realisable as the symmetry groups of the potential:

$$
\mathbb{Z}_{2}, \quad \mathbb{Z}_{3}, \quad \mathbb{Z}_{4}, \quad \mathbb{Z}_{2} \times \mathbb{Z}_{2}, \quad \mathrm{U}(1), \quad \mathrm{U}(1) \times \mathbb{Z}_{2}, \quad \mathrm{U}(1) \times \mathrm{U}(1)
$$

The only finite Abelian group that is not contained in any maximal torus in $\mathrm{PSU}(3)$ is $\mathbb{Z}_{3} \times \mathbb{Z}_{3}$. It turns out that the $\mathbb{Z}_{3} \times \mathbb{Z}_{3}$-symmetric potential is symmetric under the $\left(\mathbb{Z}_{3} \times\right.$ $\left.\mathbb{Z}_{3}\right) \rtimes \mathbb{Z}_{2}$ group which is non-Abelian. Therefore, according to our definition the symmetry group $\mathbb{Z}_{3} \times \mathbb{Z}_{3}$ is not realisable. However, it needs to be considered when constructing the realisable non-Abelian groups out of the Abelian ones, which is done in the next subsection.

\section{A.2 Non-Abelian symmetries}

To exhaust the list of finite non-Abelian subgroups $G \subset \mathrm{PSU}(3)$, one needs to find all finite Abelian subgroups $A \subset \operatorname{PSU}(3)$ :

$$
A: \quad \mathbb{Z}_{2}, \quad \mathbb{Z}_{3}, \quad \mathbb{Z}_{4}, \quad \mathbb{Z}_{2} \times \mathbb{Z}_{2}, \quad \mathbb{Z}_{3} \times \mathbb{Z}_{3}
$$

which was described in the previous subsection.

Note that the order of all Abelian subgroups $|A|$ has two prime divisors, 2 and 3. The order of all non-Abelian groups $|G|$ can therefore only have the same two prime divisors (Cauchy's theorem). This means that the group $G$ is solvable (Burnside's theorem) and contains a normal self-centralizing Abelian subgroup $A$ from the above list (A.16). Therefore, all non-Abelian subgroups $G \subset \mathrm{PSU}(3)$ can be constructed by extensions of $A$ by a subgroup of $A u t(A)[18]$ :

$$
G / A \rightarrow \operatorname{Aut}(A), \quad \text { where } \quad A \triangleleft G .
$$

Let us check the automorphisms of each Abelian subgroup $A \subset \mathrm{PSU}(3)$ and the resulting $G$;

- $\operatorname{Aut}\left(\mathbb{Z}_{2}\right)=1$, therefore $G=\mathbb{Z}_{2}$ which is an Abelian group already considered in (A.16).

- $\operatorname{Aut}\left(\mathbb{Z}_{3}\right)=\mathbb{Z}_{2}$, therefore $G$ is either $\mathbb{Z}_{6}$ or $D_{6} \simeq \mathbb{Z}_{3} \rtimes \mathbb{Z}_{2}$. However, $\mathbb{Z}_{6}$ is an Abelian group which does not appear in (A.16) and as a result is not realisable.

- $\operatorname{Aut}\left(\mathbb{Z}_{4}\right)=\mathbb{Z}_{2}$, therefore $G$ is either $D_{8} \simeq \mathbb{Z}_{4} \rtimes \mathbb{Z}_{2}$ or $Q_{8}$. However, a $Q_{8}$-symmetric potential is automatically symmetric under a continuous group of phase rotations and hence non-realisable.

- $\operatorname{Aut}\left(\mathbb{Z}_{2} \times \mathbb{Z}_{2}\right)=S_{3}$, therefore $G$ is $D_{8} \simeq\left(\mathbb{Z}_{2} \times \mathbb{Z}_{2}\right) \times \mathbb{Z}_{2}$ or $A_{4} \simeq\left(\mathbb{Z}_{2} \times \mathbb{Z}_{2}\right) \rtimes \mathbb{Z}_{3}$ or $S_{4} \simeq\left(\mathbb{Z}_{2} \times \mathbb{Z}_{2}\right) \rtimes S_{3}$, where all three groups are realisable.

- $\operatorname{Aut}\left(\mathbb{Z}_{3} \times \mathbb{Z}_{3}\right)=G L_{2}(3)$, therefore $G$ is either $\Delta(54) / \mathbb{Z}_{3} \simeq\left(\mathbb{Z}_{3} \times \mathbb{Z}_{3}\right) \rtimes \mathbb{Z}_{2}$ or $\Sigma(36) \simeq$ $\left(\mathbb{Z}_{3} \times \mathbb{Z}_{3}\right) \rtimes \mathbb{Z}_{4}$, and both groups are realisable. 
Therefore, we arrive at the full list of non-Abelian subgroups of PSU(3) realisable as symmetry groups of a $3 \mathrm{HDM}$ potential:

$$
D_{6}, \quad D_{8}, \quad A_{4}, \quad S_{4}, \quad \Delta(54) / \mathbb{Z}_{3}, \quad \Sigma(36) .
$$

\section{B Orbit space}

The general renormalisable scalar potential of NHDMs is a combination of gauge-invariant bilinears $\left(\phi_{a}^{\dagger} \phi_{b}\right)$, written compactly as [28-30]

$$
V=Y_{a b}\left(\phi_{a}^{\dagger} \phi_{b}\right)+Z_{a b c d}\left(\phi_{a}^{\dagger} \phi_{b}\right)\left(\phi_{c}^{\dagger} \phi_{d}\right)
$$

The space of electroweak-gauge orbits of Higgs fields (the orbit space) was first represented via bilinears for 2HDMs, [31-37], and then extended to $N$ doublets in [13].

The orbit space can be represented as a certain algebraic manifold in the Euclidean space $\mathbb{R}^{N^{2}}$ of the bilinears. It is convenient to group these bilinears in the following way:

$$
r_{0}=\sqrt{\frac{N-1}{2 N}} \sum_{a} \phi_{a}^{\dagger} \phi_{a}, \quad r_{i}=\sum_{a, b} \phi_{a}^{\dagger} \lambda_{a b}^{i} \phi_{b}, \quad i=1, \ldots, N^{2}-1
$$

where $\lambda^{i}$ are the generators of $\mathrm{SU}(N)$. In terms of the bilinears, the Higgs potential can then be written as:

$$
V=-M_{0} r_{0}-M_{i} r_{i}+\frac{1}{2} \Lambda_{00} r_{0}^{2}+\Lambda_{0 i} r_{0} r_{i}+\frac{1}{2} \Lambda_{i j} r_{i} r_{j}
$$

The orbit space in NHDMs was characterised algebraically and geometrically in [13] and obeys the following conditions

$$
r_{0} \geq 0, \quad \frac{N-2}{2(N-1)} r_{0}^{2} \leq \vec{r}^{2} \leq r_{0}^{2},
$$

lying between two forward cones. It is interesting to note that in the case of $2 \mathrm{HDMs}(N=2)$ the inner cone disappears and the orbit space fills the entire forward cone $\left(0 \leq \vec{r}^{2} \leq r_{0}^{2}\right)$.

It is known that the neutral vacua always lie on the surface of the outer cone $\vec{r}^{2}=r_{0}^{2}$, and the charge-breaking vacua occupy a certain region strictly inside the cone, $\vec{r}^{2}<r_{0}^{2}$.

In the particular case of 3HDMs, the bilinears are as follows:

$$
\begin{aligned}
& r_{0}=\frac{\left(\phi_{1}^{\dagger} \phi_{1}\right)+\left(\phi_{2}^{\dagger} \phi_{2}\right)+\left(\phi_{3}^{\dagger} \phi_{3}\right)}{\sqrt{3}}, r_{3}=\frac{\left(\phi_{1}^{\dagger} \phi_{1}\right)-\left(\phi_{2}^{\dagger} \phi_{2}\right)}{2}, r_{8}=\frac{\left(\phi_{1}^{\dagger} \phi_{1}\right)+\left(\phi_{2}^{\dagger} \phi_{2}\right)-2\left(\phi_{3}^{\dagger} \phi_{3}\right)}{2 \sqrt{3}} \\
& r_{1}=\operatorname{Re}\left(\phi_{1}^{\dagger} \phi_{2}\right), \quad r_{4}=\operatorname{Re}\left(\phi_{3}^{\dagger} \phi_{1}\right), \quad r_{6}=\operatorname{Re}\left(\phi_{2}^{\dagger} \phi_{3}\right), \\
& r_{2}=\operatorname{Im}\left(\phi_{1}^{\dagger} \phi_{2}\right), \quad r_{5}=\operatorname{Im}\left(\phi_{3}^{\dagger} \phi_{1}\right), \quad r_{7}=\operatorname{Im}\left(\phi_{2}^{\dagger} \phi_{3}\right) .
\end{aligned}
$$

The orbit space in $3 \mathrm{HDM}$ is defined by

$$
r_{0} \geq 0, \quad \frac{1}{4} r_{0}^{2} \leq \vec{r}^{2} \leq r_{0}^{2}, \quad \sqrt{3} d_{i j k} r_{i} r_{j} r_{k}=\frac{3 \vec{r}^{2}-r_{0}^{2}}{2} r_{0},
$$

where $d_{i j k}$ is the fully symmetric $\mathrm{SU}(3)$ tensor [13]. 


\section{The geometric minimisation method}

The geometric minimisation method was developed for highly symmetric potentials in [24], namely for $A_{4}$ and $S_{4}$ symmetric 3 HDMs. Here we briefly introduce the method and show the results in $A_{4}, S_{4}, \Delta(54) / Z_{3}$ and $\Sigma(36)$ symmetric $3 \mathrm{HDM}$ potentials.

As discussed in appendix B, a $3 \mathrm{HDM}$ scalar potential can be written as:

$$
V=-M_{0} r_{0}-M_{i} r_{i}+\frac{1}{2} \Lambda_{00} r_{0}^{2}+\Lambda_{0 i} r_{0} r_{i}+\frac{1}{2} \Lambda_{i j} r_{i} r_{j} .
$$

The approach in [24] is applicable to potentials with sufficiently high symmetry so that $M_{i}=0$, which is a characteristic of the so called frustrated symmetries [16].

With $M_{i}=0$, the potential can be generically written as

$$
V=-M_{0} r_{0}+r_{0}^{2} \sum_{i=0}^{k} \Lambda_{i} x_{i} \quad\left(\text { where } \quad x_{i}=\frac{r_{i} r_{j}}{r_{0}^{2}}, \quad x_{0}=1\right)
$$

with $k$ different quartic terms where $k$ is usually small for highly symmetric potentials.

We now calculate all $x_{i}$, for all possible values of $r$ 's inside the orbit space, which will fill a certain region in the space $\mathbb{R}^{k}$. Therefore, this region, denoted by $\Gamma$, is the orbit space fitted into the $x_{i}$ space.

Minimisation of the potential is done by constructing the geometric shape of $\Gamma$, in several steps;

- The potential (C.2) is a linear function of $x_{i}$, therefore a "steepest descent" direction $\vec{n}=-\left(\Lambda_{1}, \ldots, \Lambda_{k}\right)$ can be introduced in which the potential reduces its value the fastest.

- The potential can then be rewritten as

$$
V=-M_{0} r_{0}+r_{0}^{2}\left(\Lambda_{0}-\vec{n} \vec{x}\right) .
$$

- The points sitting the farthest in the direction of $\vec{n}$ represent the minima of the potential.

- Having identified these points $x_{i}$, we find their realisations in terms of fields.

We present the results of applying this method to the frustrated symmetries in the list (2.11) in the following sections.

\section{C.1 A A $^{- \text {symmetric potential }}$}

Recall that the $A_{4}$ symmetric potential in terms of the fields has the following form

$$
\begin{aligned}
V_{A_{4}}= & -\mu^{2}\left[\left(\phi_{1}^{\dagger} \phi_{1}\right)+\left(\phi_{2}^{\dagger} \phi_{2}\right)+\left(\phi_{3}^{\dagger} \phi_{3}\right)\right]+\lambda_{11}\left[\left(\phi_{1}^{\dagger} \phi_{1}\right)+\left(\phi_{2}^{\dagger} \phi_{2}\right)+\left(\phi_{3}^{\dagger} \phi_{3}\right)\right]^{2} \\
& +\lambda_{12}\left[\left(\phi_{1}^{\dagger} \phi_{1}\right)\left(\phi_{2}^{\dagger} \phi_{2}\right)+\left(\phi_{2}^{\dagger} \phi_{2}\right)\left(\phi_{3}^{\dagger} \phi_{3}\right)+\left(\phi_{3}^{\dagger} \phi_{3}\right)\left(\phi_{1}^{\dagger} \phi_{1}\right)\right] \\
& +\lambda_{12}^{\prime}\left(\left|\phi_{1}^{\dagger} \phi_{2}\right|^{2}+\left|\phi_{2}^{\dagger} \phi_{3}\right|^{2}+\left|\phi_{3}^{\dagger} \phi_{1}\right|^{2}\right)+\lambda_{1}\left[\left(\phi_{1}^{\dagger} \phi_{2}\right)^{2}+\left(\phi_{2}^{\dagger} \phi_{3}\right)^{2}+\left(\phi_{3}^{\dagger} \phi_{1}\right)^{2}\right]+\text { h.c. }
\end{aligned}
$$


The potential is rewritten using the definition of $r_{i} \mathrm{~s}$ in $3 \mathrm{HDMs}$ in eq. (B.5):

$$
\begin{aligned}
V= & -\left(\sqrt{3} \mu^{2}\right) r_{0}+\left(\frac{9 \lambda_{11}+3 \lambda_{12}}{2}\right) r_{0}^{2}+\left(\lambda_{12}^{\prime}+2 \operatorname{Re} \lambda_{1}\right)\left(r_{1}^{2}+r_{4}^{2}+r_{6}^{2}\right) \\
& +\left(\lambda_{12}^{\prime}-2 \operatorname{Re} \lambda_{1}\right)\left(r_{2}^{2}+r_{5}^{2}+r_{7}^{2}\right)+\left(\frac{-3 \lambda_{11}-\lambda_{12}}{2}\right)\left(r_{3}^{2}+r_{8}^{2}\right) \\
& +\left(4 i \operatorname{Im} \lambda_{1}\right)\left(r_{1} r_{2}+r_{4} r_{5}+r_{6} r_{7}\right)
\end{aligned}
$$

which could be written in the simplified form:

$$
V=-M_{0} r_{0}+r_{0}^{2}\left(\Lambda_{0}+\Lambda_{1} x+\Lambda_{2} y+\Lambda_{3} z+\Lambda_{4} t\right)
$$

where

$$
\begin{array}{llll}
M_{0}=\sqrt{3} \mu^{2}, & \Lambda_{0}=\frac{9 \lambda_{11}+3 \lambda_{12}}{2}, & \Lambda_{1}=\lambda_{12}^{\prime}+2 \operatorname{Re} \lambda_{1} \\
\Lambda_{2}=\lambda_{12}^{\prime}-2 \operatorname{Re} \lambda_{1}, & \Lambda_{3}=\frac{-3 \lambda_{11}-\lambda_{12}}{2}, & \Lambda_{4}=4 i \operatorname{Im} \lambda_{1} \\
x=\frac{r_{1}^{2}+r_{4}^{2}+r_{6}^{2}}{r_{0}^{2}}, & y=\frac{r_{2}^{2}+r_{5}^{2}+r_{7}^{2}}{r_{0}^{2}}, & z=\frac{r_{3}^{2}+r_{8}^{2}}{r_{0}^{2}}, \quad t=\frac{r_{1} r_{2}+r_{4} r_{5}+r_{6} r_{7}}{r_{0}^{2}} .
\end{array}
$$

The neutral part of the orbit space has a complicated shape [24] with its four vertices corresponding to the four neutral global minimum alignment for this potential, which are the following:

$$
(0,0,1), \quad(1,1,1), \quad\left(1, e^{i \pi / 3}, e^{-i \pi / 3}\right), \quad\left(0,1, e^{i \alpha}\right)
$$

where only the relative magnitude of VEVs is given and in each case arbitrary permutation and sign change of doublets are allowed.

The point $(0,0,1)$ becomes the global minimum of the potential (C.5) when

$$
\lambda_{11}+\lambda_{12}>0, \quad\left(2 \lambda_{12}^{\prime}+3 \lambda_{11}+3 \lambda_{12}\right)^{2}>16\left(\operatorname{Re}^{2} \lambda_{1}-\operatorname{Im}^{2} \lambda_{1}\right)
$$

in addition to the conditions in (4.11).

\section{C.2 $\mathrm{S}_{4}$-symmetric potential}

Recall the $S_{4}$-symmetric potential:

$$
\begin{aligned}
V_{S_{4}}= & -\mu^{2}\left[\left(\phi_{1}^{\dagger} \phi_{1}\right)+\left(\phi_{2}^{\dagger} \phi_{2}\right)+\left(\phi_{3}^{\dagger} \phi_{3}\right)\right]+\lambda_{11}\left[\left(\phi_{1}^{\dagger} \phi_{1}\right)+\left(\phi_{2}^{\dagger} \phi_{2}\right)+\left(\phi_{3}^{\dagger} \phi_{3}\right)\right]^{2} \\
& +\lambda_{12}\left[\left(\phi_{1}^{\dagger} \phi_{1}\right)\left(\phi_{2}^{\dagger} \phi_{2}\right)+\left(\phi_{2}^{\dagger} \phi_{2}\right)\left(\phi_{3}^{\dagger} \phi_{3}\right)+\left(\phi_{3}^{\dagger} \phi_{3}\right)\left(\phi_{1}^{\dagger} \phi_{1}\right)\right] \\
& +\lambda_{12}^{\prime}\left(\left|\phi_{1}^{\dagger} \phi_{2}\right|^{2}+\left|\phi_{2}^{\dagger} \phi_{3}\right|^{2}+\left|\phi_{3}^{\dagger} \phi_{1}\right|^{2}\right)+\lambda_{1}\left[\left(\phi_{1}^{\dagger} \phi_{2}\right)^{2}+\left(\phi_{2}^{\dagger} \phi_{3}\right)^{2}+\left(\phi_{3}^{\dagger} \phi_{1}\right)^{2}\right]+\text { h.c. }
\end{aligned}
$$

which in terms of the $r_{i}$ s has the following form:

$$
\begin{aligned}
V= & -\left(\sqrt{3} \mu^{2}\right) r_{0}+\left(\frac{9 \lambda_{11}+3 \lambda_{12}}{2}\right) r_{0}^{2}+\left(\lambda_{12}^{\prime}+2 \lambda_{1}\right)\left(r_{1}^{2}+r_{4}^{2}+r_{6}^{2}\right) \\
& +\left(\lambda_{12}^{\prime}-2 \lambda_{1}\right)\left(r_{2}^{2}+r_{5}^{2}+r_{7}^{2}\right)+\left(\frac{-3 \lambda_{11}-\lambda_{12}}{2}\right)\left(r_{3}^{2}+r_{8}^{2}\right)
\end{aligned}
$$


which could be written in the simplified form:

$$
V=-M_{0} r_{0}+r_{0}^{2}\left(\Lambda_{0}+\Lambda_{1} x+\Lambda_{2} y+\Lambda_{3} z\right)
$$

where

$$
\Lambda_{1}=\lambda_{12}^{\prime}+2 \lambda_{1}, \quad \Lambda_{2}=\lambda_{12}^{\prime}-2 \lambda_{1}
$$

and the same expression as in the $A_{4}$ case for $M_{0}, \Lambda_{0}, \Lambda_{3}$ and $x, y, z$.

The neutral part of the orbit space has the shape of a trapezoid [24] with its four vertices corresponding to the four neutral global minimum alignment for this potential, which are the following:

$$
(0,0,1), \quad(1,1,1), \quad\left(1, e^{i \pi / 3}, e^{-i \pi / 3}\right), \quad\left(0, e^{i \pi / 4}, e^{-i \pi / 4}\right)
$$

where in each case, arbitrary permutation and sign change of doublets are allowed.

The alignment $(0,0,1)$ becomes the global minimum of the potential (C.7) when

$$
\lambda_{11}>0, \quad \lambda_{11}+\lambda_{12}>0, \quad 3\left(\lambda_{11}+\lambda_{12}\right)>-\lambda_{12}^{\prime} \pm 2 \lambda_{1}
$$

in addition to the conditions in (4.18).

\section{C.3 $\Delta(54) / Z_{3}$-symmetric potential}

The $\Delta(54) / Z_{3}$ symmetric potential has the following form, in terms of the fields:

$$
\begin{aligned}
V_{\Delta(54) / Z_{3}=} & -\mu^{2}\left[\phi_{1}^{\dagger} \phi_{1}+\phi_{2}^{\dagger} \phi_{2}+\phi_{3}^{\dagger} \phi_{3}\right]+\lambda_{11}\left[\phi_{1}^{\dagger} \phi_{1}+\phi_{2}^{\dagger} \phi_{2}+\phi_{3}^{\dagger} \phi_{3}\right]^{2} \\
& +\lambda_{12}\left[\left(\phi_{1}^{\dagger} \phi_{1}\right)^{2}+\left(\phi_{2}^{\dagger} \phi_{2}\right)^{2}+\left(\phi_{3}^{\dagger} \phi_{3}\right)^{2}-\left(\phi_{1}^{\dagger} \phi_{1}\right)\left(\phi_{2}^{\dagger} \phi_{2}\right)-\left(\phi_{2}^{\dagger} \phi_{2}\right)\left(\phi_{3}^{\dagger} \phi_{3}\right)-\left(\phi_{3}^{\dagger} \phi_{3}\right)\left(\phi_{1}^{\dagger} \phi_{1}\right)\right] \\
& +\lambda_{12}^{\prime}\left[\left|\phi_{1}^{\dagger} \phi_{2}\right|^{2}+\left|\phi_{2}^{\dagger} \phi_{3}\right|^{2}+\left|\phi_{3}^{\dagger} \phi_{1}\right|^{2}\right] \\
& +\lambda_{1}\left[\left(\phi_{1}^{\dagger} \phi_{2}\right)\left(\phi_{1}^{\dagger} \phi_{3}\right)+\left(\phi_{2}^{\dagger} \phi_{3}\right)\left(\phi_{2}^{\dagger} \phi_{1}\right)+\left(\phi_{3}^{\dagger} \phi_{1}\right)\left(\phi_{3}^{\dagger} \phi_{2}\right)\right]+\text { h.c. }
\end{aligned}
$$

and in terms of $r_{i} \mathrm{~s}$ :

$$
\begin{aligned}
V= & -\left(\sqrt{3} \mu^{2}\right) r_{0}+\left(3 \lambda_{11}\right) r_{0}^{2}+\left(3 \lambda_{12}\right)\left(r_{3}^{2}+r_{8}^{2}\right)+\left(\lambda_{12}^{\prime}\right)\left[\left(r_{1}^{2}+r_{4}^{2}+r_{6}^{2}\right)+\left(r_{2}^{2}+r_{5}^{2}+r_{7}^{2}\right)\right] \\
& +\left(2 \operatorname{Re} \lambda_{1}\right)\left[\left(r_{1} r_{4}+r_{4} r_{6}+r_{6} r_{1}\right)+\left(r_{2} r_{5}+r_{5} r_{7}+r_{7} r_{2}\right)\right] \\
& +\left(2 i \operatorname{Im} \lambda_{1}\right)\left[r_{1} r_{5}-r_{2} r_{4}+r_{5} r_{6}-r_{4} r_{7}+r_{7} r_{1}-r_{2} r_{6}\right] \\
= & -\left(\sqrt{3} \mu^{2}\right) r_{0}+r_{0}^{2}\left[3 \lambda_{11}+3 \lambda_{12} z+\lambda_{12}^{\prime}(x+y)+2 \operatorname{Re} \lambda_{1}\left(x^{\prime}+y^{\prime}\right)+2 i \operatorname{Im} \lambda_{1} t^{\prime}\right]
\end{aligned}
$$

where

$$
\begin{aligned}
x^{\prime} & =\frac{r_{1} r_{4}+r_{4} r_{6}+r_{6} r_{1}}{r_{0}^{2}}, \quad y^{\prime}=\frac{r_{2} r_{5}+r_{5} r_{7}+r_{7} r_{2}}{r_{2}^{0}} \\
t^{\prime} & =\frac{r_{1} r_{5}-r_{2} r_{4}+r_{5} r_{6}-r_{4} r_{7}+r_{7} r_{1}-r_{2} r_{6}}{r_{0}^{2}}
\end{aligned}
$$

and the same expression as in the $A_{4}$ case for $x, y, z$. 
The points satisfying the neutral global minima conditions are the following:

$$
(0,0,1), \quad\left(0,0, e^{i \pi / 2}\right), \quad\left(0,1, e^{i \pi / 2}\right)
$$

provided

$$
\left|\operatorname{Re} \lambda_{1}\right|<\left|\lambda_{12}^{\prime}-3 \lambda_{12}\right|
$$

In each case of the minimum points, arbitrary permutation and sign change of doublets are allowed. The alignment $(0,0,1)$ becomes the global minimum of the potential (C.11) when the following conditions, in addition to (4.24), are satisfied

$$
\lambda_{11}>0, \quad \lambda_{12}>0, \quad \lambda_{12}^{\prime}>0 .
$$

\section{C.4 $\Sigma(36)$-symmetric potential}

The $\Sigma(36)$ symmetric potential has the following form, in terms of the fields:

$$
\begin{aligned}
V_{\Sigma(36)}= & -\mu^{2}\left(\phi_{1}^{\dagger} \phi_{1}+\phi_{2}^{\dagger} \phi_{2}+\phi_{3}^{\dagger} \phi_{3}\right)+\lambda_{11}\left(\phi_{1}^{\dagger} \phi_{1}+\phi_{2}^{\dagger} \phi_{2}+\phi_{3}^{\dagger} \phi_{3}\right)^{2} \\
& +\lambda_{12}^{\prime}\left(\left|\phi_{1}^{\dagger} \phi_{2}-\phi_{2}^{\dagger} \phi_{3}\right|^{2}+\left|\phi_{2}^{\dagger} \phi_{3}-\phi_{3}^{\dagger} \phi_{1}\right|^{2}+\left|\phi_{3}^{\dagger} \phi_{1}-\phi_{1}^{\dagger} \phi_{2}\right|^{2}\right)
\end{aligned}
$$

and in terms of the $r_{i} \mathrm{~s}$ :

$$
\begin{aligned}
V= & -\left(\sqrt{3} \mu^{2}\right) r_{0}+\left(3 \lambda_{11}\right) r_{0}^{2} \\
& +\left(2 \lambda_{12}^{\prime}\right)\left[\left(r_{1}^{2}+r_{4}^{2}+r_{6}^{2}\right)-\left(r_{1} r_{4}+r_{4} r_{6}+r_{6} r_{1}\right)\right. \\
& \left.\quad+\left(r_{2}^{2}+r_{5}^{2}+r_{7}^{2}\right)-\left(r_{2} r_{5}+r_{5} r_{7}+r_{7} r_{2}\right)\right] \\
= & -\left(\sqrt{3} \mu^{2}\right) r_{0}+r_{0}^{2}\left[3 \lambda_{11}+2 \lambda_{12}^{\prime}\left(x-x^{\prime}+y-y^{\prime}\right)\right] .
\end{aligned}
$$

The orbit space of this potential has a simple parabolic shape in the 3-dimensional space of $\left(r_{1}, r_{4}, r_{6}\right)$ (or equivalently in the space of $\left(r_{2}, r_{5}, r_{7}\right)$ ).

The points satisfying the neutral global minima conditions are the following:

$$
(0,0,1), \quad\left(0,0, e^{i \pi / 2}\right), \quad\left(0,1, e^{i \pi / 2}\right) .
$$

In each case of the minimum points, arbitrary permutation and sign change of doublets are allowed. The alignment $(0,0,1)$ becomes the global minimum of the potential (C.15) when the following condition, in addition to (4.31), is satisfied:

$$
\lambda_{11}>0
$$

Open Access. This article is distributed under the terms of the Creative Commons Attribution License (CC-BY 4.0), which permits any use, distribution and reproduction in any medium, provided the original author(s) and source are credited. 


\section{References}

[1] ATLAS collaboration, Observation of a new particle in the search for the Standard Model Higgs boson with the ATLAS detector at the LHC, Phys. Lett. B 716 (2012) 1 [arXiv: 1207.7214] [INSPIRE].

[2] CMS collaboration, Observation of a new boson at a mass of $125 \mathrm{GeV}$ with the CMS experiment at the LHC, Phys. Lett. B 716 (2012) 30 [arXiv:1207.7235] [INSPIRE].

[3] J.F. Gunion, H.E. Haber, G.L. Kane and S. Dawson, The Higgs hunter's guide, Front. Phys. 80 (2000) 1 [INSPIRE].

[4] D. Chung et al., The soft supersymmetry breaking Lagrangian: theory and applications, Phys. Rept. 407 (2005) 1 [hep-ph/0312378] [INSPIRE].

[5] S. Chang et al., Two Higgs doublet models for the LHC Higgs boson data at $\sqrt{s}=7$ and $8 \mathrm{TeV}$, arXiv: 1310.3374 [INSPIRE].

[6] S. Davidson and H.E. Haber, Basis-independent methods for the two-Higgs-doublet model, Phys. Rev. D 72 (2005) 035004 [Erratum ibid. D 72 (2005) 099902] [hep-ph/0504050] [INSPIRE].

[7] M. Maniatis, A. von Manteuffel and O. Nachtmann, CP violation in the general two-Higgs-doublet model: a geometric view, Eur. Phys. J. C 57 (2008) 719 [arXiv:0707.3344] [INSPIRE].

[8] C. Nishi, Physical parameters and basis transformations in the two-Higgs-doublet model, Phys. Rev. D 77 (2008) 055009 [arXiv:0712.4260] [InSPIRE].

[9] I.P. Ivanov, Minkowski space structure of the Higgs potential in 2HDM. II. Minima, symmetries and topology, Phys. Rev. D 77 (2008) 015017 [arXiv:0710.3490] [InSPIRE].

[10] R.A. Battye, G.D. Brawn and A. Pilaftsis, Vacuum topology of the two Higgs doublet model, JHEP 08 (2011) 020 [arXiv:1106.3482] [INSPIRE].

[11] A. Pilaftsis, On the classification of accidental symmetries of the two Higgs doublet model potential, Phys. Lett. B 706 (2012) 465 [arXiv:1109.3787] [INSPIRE].

[12] I.P. Ivanov, V. Keus and E. Vdovin, Abelian symmetries in multi-Higgs-doublet models, J. Phys. A 45 (2012) 215201 [arXiv:1112.1660] [INSPIRE].

[13] I. Ivanov and C. Nishi, Properties of the general NHDM. I. The orbit space, Phys. Rev. D 82 (2010) 015014 [arXiv:1004.1799] [INSPIRE].

[14] I. Ivanov, Properties of the general NHDM. II. Higgs potential and its symmetries, JHEP 07 (2010) 020 [arXiv: 1004.1802] [INSPIRE].

[15] R. Mohapatra and C. Nishi, Absence of spontaneous CP-violation in multi-Higgs doublet extension of MSSM, Phys. Rev. D 84 (2011) 095023 [arXiv:1108.1769] [InSPIRE].

[16] I.P. Ivanov and V. Keus, Frustrated symmetries in multi-Higgs-doublet models, Phys. Lett. B 695 (2011) 459 [arXiv:1007.5305] [INSPIRE].

[17] I. Ivanov and V. Keus, $Z_{p}$ scalar dark matter from multi-Higgs-doublet models, Phys. Rev. D 86 (2012) 016004 [arXiv: 1203.3426] [INSPIRE].

[18] I. Ivanov and E. Vdovin, Classification of finite reparametrization symmetry groups in the three-Higgs-doublet model, Eur. Phys. J. C 73 (2013) 2309 [arXiv:1210.6553] [INSPIRE].

[19] R. González Felipe, H. Serôdio and J.P. Silva, Models with three Higgs doublets in the triplet representations of $A_{4}$ or $S_{4}$, Phys. Rev. D 87 (2013) 055010 [arXiv: 1302.0861] [INSPIRE].

[20] S. Morisi, E. Peinado, Y. Shimizu and J. Valle, Relating quarks and leptons without grand-unification, Phys. Rev. D 84 (2011) 036003 [arXiv:1104.1633] [INSPIRE]. 
[21] S. King, S. Moretti and R. Nevzorov, Theory and phenomenology of an exceptional supersymmetric Standard Model, Phys. Rev. D 73 (2006) 035009 [hep-ph/0510419] [INSPIRE].

[22] S. King, S. Moretti and R. Nevzorov, Exceptional supersymmetric Standard Model, Phys. Lett. B 634 (2006) 278 [hep-ph/0511256] [INSPIRE].

[23] R. Howl and S. King, Solving the flavour problem in supersymmetric Standard Models with three Higgs families, Phys. Lett. B 687 (2010) 355 [arXiv:0908. 2067] [INSPIRE].

[24] A. Degee, I. Ivanov and V. Keus, Geometric minimization of highly symmetric potentials, JHEP 02 (2013) 125 [arXiv: 1211.4989] [INSPIRE].

[25] J. Unwin, Vacuum stability and the Cholesky decomposition, Eur. Phys. J. C 71 (2011) 1663 [arXiv: 1102.2896] [INSPIRE].

[26] B. Grzadkowski, O. Ogreid, P. Osland, A. Pukhov and M. Purmohammadi, Exploring the CP-violating inert-doublet model, JHEP 06 (2011) 003 [arXiv: 1012.4680] [INSPIRE].

[27] H. Ishimori et al., Non-Abelian discrete symmetries in particle physics, Prog. Theor. Phys. Suppl. 183 (2010) 1 [arXiv:1003.3552] [INSPIRE].

[28] L. Lavoura and J.P. Silva, Fundamental CP-violating quantities in a $\mathrm{SU}(2) \times \mathrm{U}(1)$ model with many Higgs doublets, Phys. Rev. D 50 (1994) 4619 [hep-ph/9404276] [INSPIRE].

[29] F. Botella and J.P. Silva, Jarlskog-like invariants for theories with scalars and fermions, Phys. Rev. D 51 (1995) 3870 [hep-ph/9411288] [INSPIRE].

[30] G.C. Branco, L. Lavoura and J.P. Silva, CP violation, Int. Ser. Monogr. Phys. 103 (1999) 1 [INSPIRE].

[31] C. Nishi, CP violation conditions in $N$-Higgs-doublet potentials, Phys. Rev. D 74 (2006) 036003 [Erratum ibid. D 76 (2007) 119901] [hep-ph/0605153] [INSPIRE].

[32] P. Ferreira, H.E. Haber, M. Maniatis, O. Nachtmann and J.P. Silva, Geometric picture of generalized-CP and Higgs-family transformations in the two-Higgs-doublet model, Int. J. Mod. Phys. A 26 (2011) 769 [arXiv:1010.0935] [InSPIRE].

[33] I. Ivanov, Two-Higgs-doublet model from the group-theoretic perspective, Phys. Lett. B 632 (2006) 360 [hep-ph/0507132] [InSPIRE].

[34] I. Ivanov, Minkowski space structure of the Higgs potential in 2HDM, Phys. Rev. D 75 (2007) 035001 [Erratum ibid. D 76 (2007) 039902] [hep-ph/0609018] [INSPIRE].

[35] I.P. Ivanov, Minkowski space structure of the Higgs potential in 2HDM. II. Minima, symmetries and topology, Phys. Rev. D 77 (2008) 015017 [arXiv: 0710.3490] [INSPIRE].

[36] G. Sartori and G. Valente, Allowed and observable phases in two Higgs doublet Standard Models, Ph.D. thesis, http://www.ub.uni-heidelberg.de/archiv/4803, University Heidelberg, Heidelberg Germany (2004) [hep-ph/0304026] [INSPIRE].

[37] M. Maniatis, A. von Manteuffel, O. Nachtmann and F. Nagel, Stability and symmetry breaking in the general two-Higgs-doublet model, Eur. Phys. J. C 48 (2006) 805 [hep-ph/0605184] [INSPIRE]. 